Visualização exploratória de dados volumétricos multivalorados variantes no tempo 

SERVIÇO DE PÓS-GRADUAÇÃO DO ICMC-USP

Data de Depósito: 07 de dezembro de 2012

Assinatura:

\title{
Visualização exploratória de dados volumétricos multivalorados variantes no tempo
}

\author{
Thiago Silva Reis Santos
}

Orientadora: Profa. Dra. Maria Cristina Ferreira de Oliveira

\begin{abstract}
Dissertação apresentada ao Instituto de Ciências Matemáticas e de Computação - ICMC-USP, como parte dos requisitos para obtenção do título de Mestre em Ciências - Ciências de Computação e Matemática Computacional. VERSÃO REVISADA
\end{abstract}


Ficha catalográfica elaborada pela Biblioteca Prof. Achille Bassi e Seção Técnica de Informática, ICMC/USP, com os dados fornecidos pelo(a) autor(a)

Silva Reis Santos, Thiago
Visualizaça exploratória de dados volumétricos
multivalorados variantes no tempo / Thiago Silva
Reis Santos; orientadora Maria Cristina Ferreira de
Oliveira. -- São Carlos, 2012 .
75 p.
Dissertação (Mestrado - Programa de Pós-Graduação en
Ciências de Computação e Matemática Computacional) --
Instituto de Ciências Matemáticas e de Computação,
Universidade de São Paulo, 2012.
l. projeção multidimensional. 2. visualização da
informação. 3. visualização científica. 4. simulações
por computador. I. Ferreira de Oliveira, Maria
Cristina, orient. II. Título.


"O Senhor é meu pastor e nada me faltará" (Salmo 22,1) 


\section{Agradecimentos}

Chega ao final mais uma preciosa etapa da minha vida: o mestrado! Durante a caminhada, enfrentei obstáculos e desafios, mas venci! A realização deste sonho só foi possível graças:

À Deus pelo amor infinito, presença constante em minha vida, por todas as bênçãos e graças e por ter colocado em meu caminho pessoas maravilhosas, fundamentais para que este momento acontecesse. Muito obrigado meu Deus por tudo!!!

À minha amada esposa Aline pelo amor, incentivo e companheirismo dedicados a mim; especialmente pelo início do mestrado que coincidiu com o nosso casamento. Por muitas vezes foi difícil superar a distância, mas graças a Deus o nosso amor foi maior e juntos superamos e vencemos essa fase. Te amo demais e nunca vou esquecer o que você tem feito por mim!

Aos meus sogros Belarmino e Rosa que se fizeram nesse tempo verdadeiros pais para mim, sempre presentes, incentivando, apoiando e estando junto em tudo o que foi necessário, principalmente nos momentos mais difíceis. Sem vocês eu não terei conseguido! Agradeço também à minha cunhada Karine e seu esposo Jadson, como também a toda família de Aline pelo apoio. Muito obrigado!!!

Aos meus pais Célio e Ailta por me ensinarem a importância do estudo. Aos meus irmãos Aline e Ailton pelo apoio e incentivo.

À minha orientadora Maria Cristina pela oportunidade, paciência e por acreditar no meu trabalho.

A todos os professores do ICMC, em especial Fernando e Rosane. Não esquecendo dos meus professores da UFS, em especial Leonardo e Davi.

Aos amigos conquistados e aos colegas de laboratório pela ajuda indispensável durante os anos de mestrado, em especial ao Christian, Eduardo, Vinícius, Valéria e Alceu.

Às agências CAPES e FAPESP pelo apoio financeiro.

Enfim, a todos que fazem parte da minha vida e contribuíram para este momento. 


\section{Resumo}

SANTOS, Thiago S. Reis. Visualização Exploratória de Volumes de Dados Multimodais Apoiada por Técnicas de Projeção Multidimensional. 2012. Dissertação (Mestrado) - Instituto de Ciências Matemática e de Computação, Universidade de São Paulo, São Carlos, 2012 .

Simulações por computador permitem reduzir custo e, muitas vezes, realizar experimentos que na vida real seriam impraticáveis, ou por questões ambientais (explosões nucleares), ou por fatores que estão fora do controle do ser humano (colisões entre estrelas). Entretanto, é muito difícil manipular e analisar as centenas de gigabytes, ou mesmo terabytes, que tais simulações produzem como resultado. Os trabalhos que lidam com tais conjuntos de dados, tipicamente, empregam tanto técnicas de visualização científica como técnicas de visualização da informação, em geral refletindo o comportamento dos dados em um único instante de tempo. Entretanto, a análise da evolução temporal e a disponibilização de representações visuais integradas ainda é um grande desafio. Esse trabalho introduz diversas estratégias buscando tratar estes problemas, as quais tem em comum a utilização de projeções multidimensionais para apoiar a análise exploratória dos de dados, tanto em um instante de tempo específico, como ao longo da evolução temporal. O objetivo é favorecer a localização de grupos de elementos com comportamento similar e acompanhar sua evolução ao longo da simulação. Uma das estratégias introduzidas resume o comportamento temporal dos dados multidimensionais em uma única visualização, o que permite rastrear as entidades com comportamento similar e analisá-las ao longo da simulação.

Palavras-chave: Simulações por computador, projeção multidimensional, visualização da informação e visualização científica. 


\section{Abstract}

SANTOS, Thiago S. Reis. Exploratory Visualization of Multimodal Volume Data Supported by Multidimensional Projection Techniques. 2012. Dissertation (Master) - Institute of Mathematics and Computer Sciences, University of São Paulo, São Carlos, 2012.

Computer simulations of physical phenomena allow reducing costs and studying behavior that would be unfeasible to observe in real life situations, either due to environmental limitations, e.g., a nuclear explosion, or due to factors that are beyond human control (e.g., collisions between stars). Millions of primitives (voxels, vertices or particle) may be required to accurately capture system behavior, thus generating very large data sets that are typically time-varying and multidimensional, as multiple simulation variables describe each primitive. Therefore, analyzing the hundreds of gigabytes or even terabytes resulting from these simulations remains a challenge. Current solutions that handle this type of data usually rely on Scientific or Information Visualization techniques, but typically revealing data behavior at a particular time instant. It remains a major challenge to provide visualizations capable of assisting analysts trying to inspect and understand behavior along the temporal domain. This work is an attempt in this direction, introducing several strategies to handle these problems. They have in common the use of multidimensional projection techniques to support exploratory analysis of simulation data, both at specific time instants and along the simulation as a whole. The goal is to favor the perception of groups of elements showing similar behavior and track their temporal evolution. One of the strategies introduced summarizes, in a single visual representation, the temporal behavior of the multidimensional data space, thus allowing analysts to identify and analyze the entities with similar behavior along the simulation.

Keywords: Computer simulations, multidimensional projection, information visualization and scientific visualization. 
1 Introdução 1

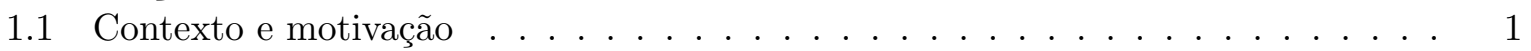

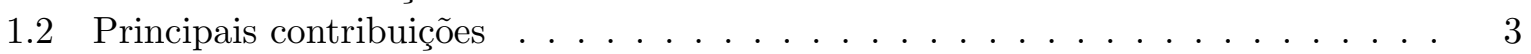

1.3 Organização da dissertação $\ldots \ldots \ldots \ldots \ldots \ldots \ldots \ldots$

2 Visualização de dados multidimensionais $\quad 5$

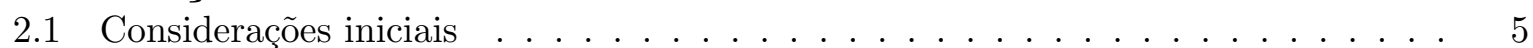

2.2 Matriz de gráficos de dispersão . . . . . . . . . . . . . . . . . . 6

2.3 Coordenadas Paralelas . . . . . . . . . . . . . . . . . . . 7

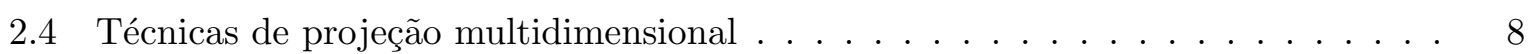

2.4.1 Análise de componentes principais $(\mathrm{PCA}) \ldots \ldots \ldots$. . . . . . 8

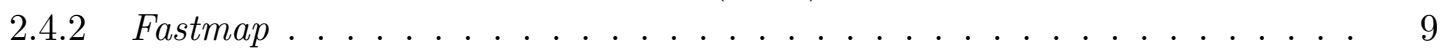

2.4 .3 Force scheme $(\mathrm{FS}) \ldots \ldots \ldots \ldots$

2.4 .4 Sammon's mapping $(\mathrm{SM}) \ldots \ldots \ldots \ldots \ldots$

2.4.5 Part-linear multidimensional projection (PLMP) . . . . . . . . . . 11

2.4.6 Least square projection (LSP) . . . . . . . . . . . . . . . . . . . . 12

2.4.7 Local affine multidimensional projection (LAMP) . . . . . . . . . . . . . . 13

2.5 Avaliando a qualidade das projeções . . . . . . . . . . . . . . . . . 13

2.6 Técnicas de agrupamento . . . . . . . . . . . . . . . . . . . . 14

2.6.1 K-Means e variações . . . . . . . . . . . . . . . . . . 15

$2.6 .2 \quad$ X-Means e variações . . . . . . . . . . . . . . . . . . . 17

2.7 Avaliando a qualidade de agrupamentos . . . . . . . . . . . . . . . . . . 18

2.8 Considerações finais . . . . . . . . . . . . . . . . . . . . . . . . . . . 19

3 Visualização de volumes variantes no tempo 21

3.1 Considerações iniciais . . . . . . . . . . . . . . . . . . . 21

3.2 Visualização de malhas volumétricas . . . . . . . . . . . . . . . . . . . 22

3.3 Visualização de volumes de partículas . . . . . . . . . . . . . . . 26

3.4 Considerações finais . . . . . . . . . . . . . . . . . . . . . . . . . 29

4 Visualização de malhas volumétricas variantes no tempo apoiada por projeções 31

4.1 Considerações iniciais . . . . . . . . . . . . . . . . . . . . . . . . . 31 
4.2 Investigação temporal de malhas volumétricas . . . . . . . . . . . . . . . . . . . 32

4.2 .1 Explorando agrupamentos . . . . . . . . . . . . . . . . . 32

4.2.2 Explorando o comportamento temporal . . . . . . . . . . . . . . . 33

4.3 Investigação temporal de campos vetoriais . . . . . . . . . . . . . . . 35

4.3 .1 Extração do vetor de características . . . . . . . . . . . . . 36

4.4 Visualização exploratória: furacão Isabel . . . . . . . . . . . . . . . . . . 36

4.5 Visualização exploratória: bomba hidráulica . . . . . . . . . . . . . . . 40

4.6 Considerações finais . . . . . . . . . . . . . . . . . . 43

5 Visualização de volumes de partículas variantes no tempo apoiada por proje$\begin{array}{lc}\text { ções } & 49\end{array}$

5.1 Considerações iniciais . . . . . . . . . . . . . . . . . . . . . . . . . . . 49

5.2 Geração das streamfeatures . . . . . . . . . . . . . . . . . 50

5.3 Investigação via streamfeatures . . . . . . . . . . . . . . . . 51

5.4 Considerações finais . . . . . . . . . . . . . . . . . 61

6 Conclusões $\quad 63$

6.1 Contribuições . . . . . . . . . . . . . . . . . . . . . . . 63

6.2 Limitações . . . . . . . . . . . . . . . . . . . . . . . . . . . . 64

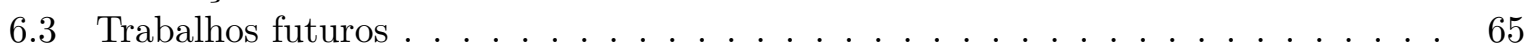

$\begin{array}{ll}\text { Referências Bibliográficas } & 67\end{array}$

$\begin{array}{ll}\text { A Viscontest } 2011 & 73\end{array}$

$\begin{array}{ll}\text { B IVAPP } 2013 & 75\end{array}$ 


\section{Lista de Figuras}

2.1 Exemplo da matriz de gráficos de dispersão para um conjunto de dados com 12

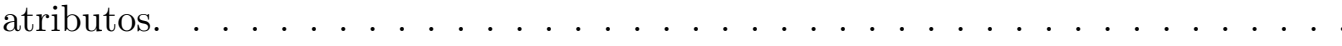

2.2 Exemplo do uso das coordenadas paralelas com um conjunto de dados de 31.703 elementos e 12 atributos. . . . . . . . . . . . . . . . . . 8

2.3 Ilustração da técnica Fastmap. Fonte: (Faloutsos e Lin, 1995) . . . . . . . . . . . 10

2.4 Ilustração da curva de estresse. . . . . . . . . . . . . . . . . . . . . . 14

2.5 Ilustração do gráfico da preservação da vizinhaça. Observa-se que a técnica representada pela linha vermelha foi capaz de preservar melhor a vizinhaça. . . . 15

3.1 Esquerda: pressão e fluído - centro: rotação - direita: vórtices. Fonte: (Schafhitzel e Weiskopf, 2004) . . . . . . . . . . . . . . . . . . . . 23

3.2 Resultado do rastreamento de uma característica. Seis instantes de tempo do intervalo entre 50 até 74 são apresentados. A característica de interesse é renderizada em vermelho. Fonte: (Tzeng, 2005). . . . . . . . . . . . . . . .

3.3 Visão de quatro componentes coordenados: histograma, gráfico de dispersão, visão de fatia e visão de fatia pseudo-colorida. Fonte: (Blaas et al., 2007) . . . . 24

3.4 Representações gráficas resultantes da abordagem definida por Linsen et al. (2008). 25

3.5 Demonstração da aplicação da técnica de Daniels et. al. sobre o conjunto de dados da simulação das correntes marítmas ao longo da costa do pacífico. No lado esquerdo a projeção do espaço de atributos colorida pelo usuário. Com as atribuições das cores no espaço de atribuitos é feito o mapeamento de textura no campo vetorial. Fonte: (Daniels et al., 2010) . . . . . . . . . . . . . . . . .

3.6 De cima para baixo: coordenadas paralelas de um conjunto de dados sintético, coordenadas paralelas reordenadas com a aplicação da técnica de Ferdosi et al. Da esquerda para direita: matriz de gráficos de dispersão sobre o mesmo conjunto de dados, matriz de gráficos de dispersão com a aplicação da técnica. Fonte: (Ferdosi e Roerdink, 2011) . . . . . . . . . . . . . . . . . . . .

3.7 Visualização de um instante de tempo da simulação de um acelerador de partículas. Fonte: (Co et al., 2005) . . . . . . . . . . . . . . . . . 8 10 4

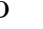


3.8 Visualização do espaço dual. Em cada par do espaço dual, a imagem a esquerda refere-se ao espaço de fase e a imagem a direta ao espaço físico. No espaço de fase, o eixo vertical corresponde a temperatura, já o eixo horizontal a fração de mistura de um conjunto de dados de simulação de combustível. Há seis pares do espaço dual, referente à aplicação da técnica de agrupamento e à manipulação pelo usuário. Fonte: (Wei et al., 2012) . . . . . . . . . . . . . . . . . . .

4.1 Exemplo de configuração dos intervalos de variação de deslocamento com 6 faixas. Os intervalos são delimitados pelos limiares indicados nas circunferências exibidas à esquerda. O raio da faixa pode ser modificado interagindo com barra de rolagem a direita . . . . . . . . . . . . . . . . . .

4.2 Matriz de gráficos de dispersão dos 16 atributos do conjunto de dados do furacão. Em destaque os atributos que aparentam possuir uma correlação linear. . . . . . .

4.3 Na coluna à esquerda tem-se as projeções em quatro instantes de tempo $(05,15,25$ e 35). Os pontos destacados nas projeções correspondem a um dos agrupamentos localizados pelo $X$-means. Na coluna do meio tem-se as Coordenadas Paralelas para o agrupamento destacado, e na coluna à direita tem-se a sua representação no espaço do objeto. . . . . . . . . . . . . . . . . .

4.4 Na coluna à esquerda tem-se as projeções em quatro instantes de tempo $(05,15$, 25 e 35). Os pontos destacados nas projeções correspondem aos elementos da faixa B (variação entre $4 \%$ e $8 \%$ ). Na coluna do meio tem-se as Coordenadas Paralelas do agrupamento destacado. Na coluna à direita tem-se o espaço do objeto do agrupamento. . . . . . . . . . . . . . . . . .

4.5 Matriz do gráfico de dispersão do conjunto de dados extraído das streamlines geradas para a simulação da bomba hidráulica (Viscontest 2011). O conjunto possui 40 atributos, gerando uma matriz 40x40 . . . . . . . . . . . .

4.6 Na coluna à esquerda tem-se as projeções referentes a quatro instantes de tempo $(10,30,50$ e 70$)$. Os pontos destacados nas projeções correspondem aos elementos de um dos agrupamentos. Na coluna do meio tem-se as Coordenadas Paralelas do agrupamento destacado. Na coluna à direita tem-se a representação do espaço do objeto (visualização das streamlines) do agrupamento destacado. . . . . . . . .

4.7 Na coluna à esquerda tem-se as projeções em quatro instantes de tempo $(11,31$, 51 e 71). Os pontos destacados nas projeções correspondem aos elementos da faixa F (variação além de $25 \%$ ). Na coluna do meio tem-se as Coordenadas Paralelas do agrupamento destacado. Na coluna à direita tem-se o espaço do objeto do

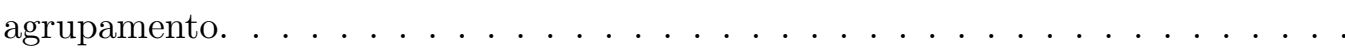

5.1 Streamfeatures (a) e os eixos 4-dimensionais (b) da simulação mini dump. . . . . 51

5.2 Pipeline para geração das streamfeatures. . . . . . . . . . . . . . . 52

5.3 Valores de estresse computados com 15 técnicas de projeção multidimensional da simulação mini dump. . . . . . . . . . . . . . . . . . . . . .

5.4 Curva de estresse dos melhores layouts dos elementos representativos da simulação mini dump: com distância Euclidiana (acima) e distância Mahalanobis (abaixo). Observa-se que as curvas de estresse dos layouts que utilizaram a distância Mahalanobis foram capazes de uma maior preservação da relação do espaço original $(s)$ pelo projetado $(\hat{s})$, uma vez que a distribuição dos pontos jaz sobre a diagonal (em vermelho). . . . . . . . . . . . . . . . . . . . .

5.5 Gráfico da preservação da vizinhança dos três melhores resultados com as distâncias Euclidiana e Mahalanobis. . . . . . . . . . . . . . . . . . 
5.6 Agrupamentos do $X$-means nas projeções. O agrupamento rosa foi selecionado na projeção das streamfeatures (c) e os elementos correspondentes foram destacados

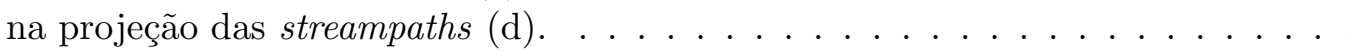

5.7 Simulação mini dump. A primeira linha corresponde a visualização das streamfeatures, já a segunda linha representa a visualização das streampaths. As primeiras seis colunas representam as visualizações em um intervalo menor (10 instante de tempo), já a última é a visualização de toda a simulação (59 instante de tempo). As cores estão mapeando o tempo, segundo a escala exibida na parte inferior da

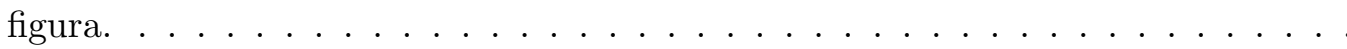

5.8 Valores de estresse computado com 15 técnicas de projeção multidimensional da simulação data explode. . . . . . . . . . . . . . . . . . . . . .

5.9 Curvas de estresse dos melhores layouts dos elementos representativos da simulação data explode: com distância Euclidiana (acima) e distância Mahalanobis (abaixo) . . . . . . . . . . . . . . . . . . .

5.10 Gráfico da preservação da vizinhança dos três melhores resultados com as distâncias Euclidiana e Mahalanobis. . . . . . . . . . . . . . . . . 58

5.11 Streamfeatures (a) e os eixos 9-dimensionais (b) da simulação data explode. . . . 58

5.12 Agrupamentos do $X$-means nas projeções. O agrupamento rosa foi selecionado na projeção das streamfeatures (c) e os elementos correspondentes foram destacados

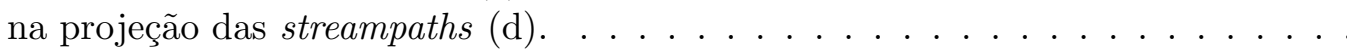

5.13 Simulação data explode. A linha superior exibe as streampaths, e a linha infeior as streamfeatures. As primeiras cinco colunas representam intervalos menores 20 instantes de tempo, já a última apresenta a visualização de toda a simulação (200 instante de tempo). As cores estão mapeando o tempo, segundo a escala exibida na parte inferior da figura. . . . . . . . . . . . . . . . . 60

5.14 Interface para a filtragem das polilinhas, neste caso das streampaths. . . . . . . . 60

5.15 Visualização das streampaths (em cima) e das streamfeatures (em baixo) das partículas filtradas nas interfaces exibidas na Figura 5.14. . . . . . . . . . . 61

5.16 Visualização das streamfeatures e das streampaths com as menores trajetórias, sendo um conjunto com 1.880 partículas filtradas pela interface exibida na Figura

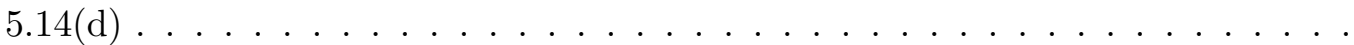




\section{Lista de Tabelas}

4.1 Atributos do furacão com a identificação dos atributos correlacionados, e dos selecionados, com a numeração final. . . . . . . . . . . . . . . . 38

4.2 Aplicação das técnicas de agrupamento no início e no meio da simulação (01 e 25). O X-means obteve os melhores resultados. . . . . . . . . . . . 38

4.3 Evolução dos pontos pertencentes ao agrupamento destacado na Figura 4.3. . . . 39

4.4 Atribuição dos limites para as quatro faixas definidas para segmentar o volume. . 39

4.5 Distribuição dos dados em relação as faixas de variação ao longo da simulação do

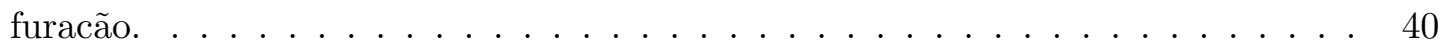

4.6 Listagem dos atributos da bomba hidráulica, com a identificação dos atributos correlacionados e a seleção para a numeração final. . . . . . . . . . . . . . . . . . 41

4.7 Aplicação das técnicas de agrupamento no início e no meio da simulação (10 e $30)$ sobre os dados projetados, usando o intervalo $[25,100]$. O X-means obteve os melhores resultados. . . . . . . . . . . . . . . . . 42

4.8 Evolução dos pontos pertencentes ao agrupamento destacado na Figura 4.6 . . 42

4.9 Atribuição dos limites para as quatro faixas definidas para segmentar o volume. . 42

4.10 Distribuição dos dados em relação as faixas de variação ao longo da simulação da Bomba Hidráulica. . . . . . . . . . . . . . . . . . . . . . . . . . 43

5.1 Atributos da simulação mini dump . . . . . . . . . . . . . . . . . . . . . . 52

5.2 Aplicação do X-means sobre as projeções das streamfeatures e streampaths. O intervalo utilizado foi $[5-50] \ldots \ldots \ldots \ldots \ldots \ldots \ldots$

5.3 Atributos da simulação data explode. . . . . . . . . . . . . . . . 56

5.4 Aplicação do X-means sobre as projeções das streamfeatures e streampaths. O intervalo utilizado foi $[5-50] \ldots \ldots \ldots \ldots \ldots$. . . . . . . . . . . . 58 


\section{Lista de Siglas}

PM Projeção Multidimensional

SciVis Visualização Científica (Scientific Visualization)

VEVVT Visualização Exploratória de Volumes Variantes no Tempo

InfoVis Visualização da Informação (Information Visualization)

CP Coordenadas Paralelas

PCA Análise de Componentes Principais

ICMC Instituto de Ciências Matemáticas e de Computação

FS Force Scheme

FDP Force-Directed Placement

LSP Least Square Projection

SM Sammon's Mapping

PLMP Part-Linear Multidimensional Projection

LAMP Local Affine Multidimensional Projection

BIC Bayesian Information Criterion

FFT Transformada Rápida de Fourier (Fast Fourier Transform)

Euc Distância Euclidiana

Mah Distância Mahalanobis

SPH Partículas Hidrodinâmica Suavizadas (Smoothed Particle Hydrodynamics) 


\subsection{Contexto e motivação}

A observação dos fenômenos naturais é um estágio fundamental do processo científico. A metodologia científica, cujas origens remontam a Aristóteles, tem como um dos fatores primordiais a observação. A visualização é, portanto, parte fundamental do desenvolvimento da ciência. A partir dela é possível a percepção e o entendimento do fenômeno estudado.

Muitas vezes, as limitações humanas impossibilitam a observação de um evento de interesse. Nesses casos, a simulação por computador torna-se a única forma de estudar determinado fenômeno. Se, por exemplo, o evento é muito grande, muito pequeno, muito rápido ou muito lento para ser observado na natureza, então apenas fenômenos simulados desses eventos podem ser observados (Paiva et al., 1999).

Em muitos dos problemas simulados, os dados têm natureza multivalorada, sendo constituídos por diversos valores escalares e vetoriais. Dados volumétricos multivalorados variantes no tempo são, tipicamente, definidos no domínio tridimensional (3D). Por coveniência, de agora em diante, tais dados serão chamados apenas de volumes multivalorados. Os volumes multivalorados são, geralmente, formados por um conjunto de arquivos que descrevem o volume em diferentes instantes de tempo, e são formados por centenas de milhares, por vezes milhões de elementos, o que torna qualquer tarefa sobre tal conjunto de dados um grande desafio.

Tais dados podem ser representados de três diferentes maneiras: organizados como volumes regulares, volumes irregulares ou volumes de partículas.

Os volumes regulares são caracterizados pela subdivisão do espaço 3D em células com geometria e topologia uniformes - tipicamente a célula à qual as informações estão associadas 
é chamada de voxel. Já os volumes irregulares são constituídos pela subdivisão do espaço 3D em células com topologia regular, mas geometria variável. As células são definidas em termos de faces e vértices e a informação está tipicamente associada aos vértices da malha. Por fim, algumas simulações descrevem o fenômeno como um conjunto de entidades discretas, denominadas partículas. Nesse caso, o conjunto de partículas posicionado no espaço 3D define o volume.

Quando se considera dados volumétricos multivalorados, além da representação espacial pode-se considerar o espaço definido pelos múltiplos atributos associados a cada primitiva (voxel, vértice ou partícula). Segundo notação introduzida na literatura recente (Blaas et al., 2007; Linsen et al., 2009; Ferdosi e Roerdink, 2011), tais dados admitem dois tipos de representação visual: representação do espaço do objeto e representação do espaço de atributos.

As técnicas de visualização que trabalham sobre a representação do espaço do objeto, ou seja, construídas a partir dos atributos espaciais, são denominadas visualizações científicas (SciVis). Exemplos de técnicas dessa natureza são o rendering volumétrico direto, extração de superfícies e visualização de streamlines (Manssour, 2002; Avila e Kitware, 2006).

Por outro lado, as técnicas de visualização que consideram apenas os múltiplos atributos escalares, associados ou não a uma posição no espaço, são chamadas de visualizações multidimensionais. Como exemplo, temos projeções multidimensionais, coordenadas paralelas e matriz de gráficos de dispersão (Telea, 2008).

Este trabalho aborda os volumes multivalorados, propondo abordagens de visualização que buscam unificar a interação do usuário com representações de ambos os espaços (objeto e atributos), potencializando a percepção humana para a descoberta do conhecimento. Objetiva-se investigar e propor visualizações interativas capazes de evidenciar as alterações ao longo do tempo, de modo a ajudar o usuário no entendimento do problema. A estratégia definida consiste em identificar e acompanhar a evolução dos elementos altamente similares, na esperança que evidenciem estruturas significativas no conjunto de dados.

Para isso, utilizam-se projeções multidimensionais para auxiliar a análise e exploração dos volumes. O arcabouço, aqui desenvolvido, inclui duas estratégias que levam em consideração a representação dos dados. A primeira é aplicada aos dados, cuja representação está vinculada a uma geometria, ou seja, volumes regulares ou volumes irregulares, os quais de agora em diante serão chamados de volumes de malhas. A segunda estratégia é aplicada nos volumes de partículas, já que tais dados não estão associados a uma região fixa do volume.

As técnicas de projeção multidimensional $(\mathrm{PM})$ vêm se destacando devido ao crescente desenvolvimento de métodos altamente precisos e escaláveis (Paulovich et al., 2010; Joia et al., 2011). Tais características são essenciais para manipular os volumes multivalorados. Outra característica das PMs é a sua capacidade de preservar a relação de similaridade entre os elementos, ou seja, elementos altamente similares no espaço multidimensional são projetados no espaço visual (no nosso caso $\mathbb{R}^{\{2,3\}}$ ) como pontos próximos no layout final, e elementos altamente dissimilares são mapeados como pontos afastados. Ademais, as PMs consideram 
simultaneamente todos os atributos escalares do conjunto de dados. Dados vetoriais também podem ser considerados, porém tais atributos são pré-processados e transformados em valores escalares, objetivando a utilização no arcabouço proposto.

Para investigar o comportamento de simulações que geram volumes de malhas, a estratégia definida baseia-se em gerar projeções do espaço de atributos (voxels ou células) em cada instante de tempo, e por meio da visualização resultante, o usuário pode interagir, objetivando explorar o volume atual, localizar os grupos similares por meio de alguma técnica de agrupamento ou mesmo visualizar as alterações na similaridade ocorrida de um instante para outro. Por fim, o usuário pode aplicar o mesmo processo em qualquer momento da simulação.

Já para a investigação do comportamento de simulações de partículas, foi desenvolvida uma estratégia diferenciada, chamada de visualização de streamfetures. Tal estratégia tenta superar uma das grandes dificuldades ao lidar com volumes multivalorados, que é a criação de uma representação visual única, capaz de sumarizar as mudanças ocorridas em toda a simulação ou em um intervalo de tempo específico. Portanto, as streamfeatures condensam em uma visualização a mudança de similaridade advindas de um intervalo de tempo, por meio do acúmulo dos sucessivos posicionamentos obtidos pela aplicação em sequência de uma projeção multidimensional. Ou seja, uma polilinha é formada conectando os sucessivos posicionamentos da partícula no espaço de atributos.

É possível observar que aplicar a mesma estratégia das streamfeatures no volume de malhas carece de interpretação semântica. Em outras palavras, no caso dos volumes de malhas, as unidades, sejam voxels ou vértices, não representam uma entidade fixa com um comportamento associado, mas a abstração de uma região do domínio espacial. Já no caso das partículas, pelo fato do livre deslocamento no espaço físico, as streamfeatures corresponderão ao rastreamento do comportamento de uma mesma entidade ao longo do tempo.

\subsection{Principais contribuições}

As principais contribuições deste trabalho de mestrado são sumarizadas a seguir:

- Definição de um arcabouço unificado para a visualização exploratória de volumes de dados multivalorados, utilizando-se de técnicas de projeção multidimensional para criar representações visuais que permitem a interação com os espaços do objeto e de atributos;

- Desenvolvimento de várias funcionalidades para interagir com volumes multivalorados;

- Desenvolvimento de uma visualizacão unificada capaz de sumarizar o comportamento temporal do espaço de atributos, denominada streamfature;

- Participação no IEEE Visualization Contest 2011, com a contribuição relatada no artigo (Santos et al., 2011) e 
- Submissão de artigo com os principais resultados relativos às streamfeatures ao IVAPP 2013 (International Conference on Information Visualization Theory and Applications).

\subsection{Organização da dissertação}

Este trabalho está organizado em seis Capítulos. No segundo Capítulo, são introduzidas técnicas para a visualização de dados multidimensionais. No terceiro Capítulo, apresentam-se trabalhos diretamente relacionados ao tema desta dissertação, que lidam com visualização de volumes variantes no tempo. No quarto Capítulo apresenta-se a estratégia para visualizar volumes de malhas, e no quinto Capítulo, o arcabouço proposto para visualizar volumes de partículas por meio das streamfeatures. No sexto Capítulo são apresentadas as conclusões, limitações e trabalhos futuros. Por fim, nos apêndices A e B tem-se uma cópia dos artigos gerados por este trabalho. 


\section{Visualização de dados multidimensionais}

\subsection{Considerações iniciais}

Os volumes multivalorados (voxels, células e partículas) podem ser descritos por múltiplos arquivos, sendo cada um deles uma grande matriz. Cada linha representa uma instância ou elemento do conjunto, e cada coluna, um atributo. Os atributos ou são valores reais ou são valores inteiros. Nesses casos, as matrizes constituídas pelos volumes são classificadas como densas. Um conjunto de dados esparsos é aquele em que a maioria dos atributos possui valor 0 (zero), e menos de 1\% dos atributos tem valor não-zero. Segundo (Tan et al., 2005), em termos práticos, a esparsidade é uma vantagem porque, normalmente, apenas os valores não-zero precisam ser armazenados e manipulados, resultando em uma redução significativa de tempo e espaço. Já um conjunto de dados densos é aquele em que os dados não são esparsos.

Apresenta-se aqui a notação matemática utilizada nesta dissertação. Considere um conjunto de dados $D=\left\{p_{1}, p_{2}, \ldots, p_{n}\right\}$, com $n$ pontos (elementos e instâncias são usados como sinônimos para pontos). Considere que esse conjunto projetado no espaço visual será chamado de $Y=$ $\left\{y_{1}, y_{2}, \ldots, y_{n}\right\}$. Cada elemento $p_{i}=\left\{t_{i 1}, t_{i 2}, \ldots, t_{i d}\right\}$ é descrito por $d$ atributos (sendo dimensão sinônimo para atributo). Considere ainda $s\left(p_{i}, p_{j}\right)$ como sendo a função de dissimilaridade entre dois pontos no espaço multidimensional $\left(\forall p_{i}, p_{j} \in D\right)$, e $\hat{s}\left(y_{i}, y_{j}\right)$ uma função de distância no espaço visual $\mathbb{R}^{\{2,3\}}$.

A função de dissimilaridade, tipicamente, gera valores entre zero e um $\left(s\left(p_{i}, p_{j}\right) \in[0,1]\right)$, que correspondem à proximidade entre dois elementos $i$ e $j$ do conjunto de dados. A partir da função de dissimilaridade, pode-se definir a função de similaridade por meio de uma transformação 
monotonicamente decrescente, por exemplo, $d\left(p_{i}, p_{j}\right)=1-s\left(p_{i}, p_{j}\right)$, onde $d\left(p_{i}, p_{j}\right)$ é a função de similaridade (Hand et al., 2001).

Segundo Tan, Steinbach e Kumar (2005), a distância Euclidiana é largamente utilizada para conjuntos de dados densos e com atributos contínuos (o que caracteriza o nosso caso), já para conjuntos de dados esparsos, geralmente utilizam-se medidas de similaridade capazes de ignorar o casamento de 0-0 (0-0 matches), como as medidas Jaccard e Cosseno. O termo distância é utilizado, neste trabalho, como sinônimo para medida de dissimilaridade.

É importante ressaltar que os atributos de um conjunto de dados podem possuir algum grau de correlação entre os valores. Para tais casos é possível utilizar uma medida de dissimilaridade capaz de aferir a proximidade com maior precisão, como a Mahalanobis,

$$
s\left(p_{i}, p_{j}\right)=\sqrt{\left(p_{i}-p_{j}\right)^{T} \times \Sigma^{-1} \times\left(p_{i}-p_{j}\right)},
$$

onde $\Sigma^{-1}$ corresponde à matriz inversa da covariância do conjunto de dados. A distância Mahalanobis é uma extensão da distância Euclidiana, porém mais cara em termos computacionais e mais apropriada para lidar com dados com algum grau de correlação.

A seguir, serão apresentadas técnicas de visualização do espaço de atributos multidimensionais, como matriz de gráficos de dispersão (2.2), as coordenadas paralelas (2.3) e as projeções multidimensionais (2.4). Ademais, apresentamos alguns métodos para verificar a qualidade das projeções multidimensionais (2.5). Por fim, discutimos alguns métodos de agrupamentos e uma forma de avaliar a qualidade dos grupos localizados pelas técnicas (2.6 e 2.7).

\subsection{Matriz de gráficos de dispersão}

Ao tratar dados multidimensionais é importante identificar os atributos que apresentam algum tipo de correlação, já que esses podem distorcer uma projeção ou mesmo gerar artefatos nas técnicas de agrupamento. Uma forma simples e conveniente de identificar correlação é por meio da matriz de gráficos de dispersão. Se um gráfico apresenta a disposição dos dados num formato de linha reta ou de uma parabólica, isso pode indicar correlação linear ou quadrática, respectivamente.

A matriz de gráficos de dispersão é uma extensão dos gráficos de dispersão para representar dados multidimensionais. A técnica exibe um gráfico de dispersão gerado para cada par de atributos, sendo os gráficos posicionados em um arranjo matricial, conforme pode ser visto na Figura 2.1. A matriz gerada é simétrica em relação à diagonal principal, e tal diagonal representa o gráfico de dispersão do mesmo atributo, resultando, geralmente, em uma linha reta.

A matriz de gráficos de dispersão possui alguns inconvenientes: à medida que o número de dimensões do conjunto de dados aumenta, ocasiona a diminuição do espaço de visualização, e em última análise, pode inviabilizar o processo; além disso, perde-se os detalhes na visualização de um único gráfico de dispersão no meio da matriz. 


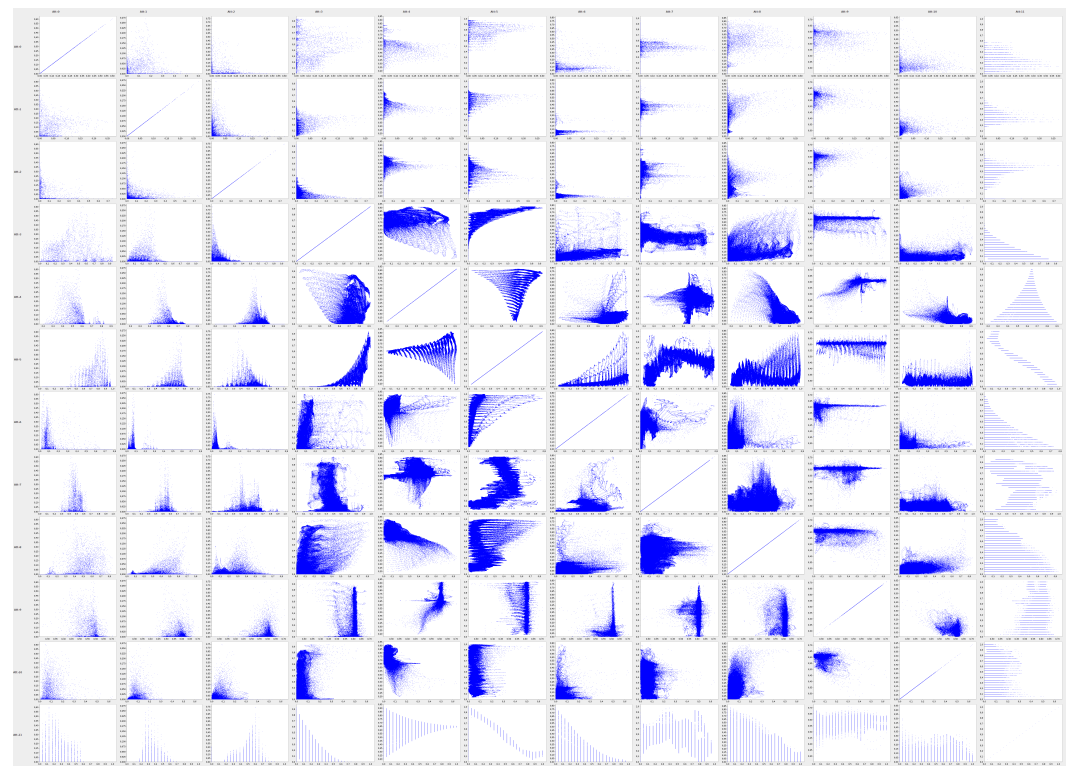

Figura 2.1: Exemplo da matriz de gráficos de dispersão para um conjunto de dados com 12 atributos.

\subsection{Coordenadas Paralelas}

A técnica de Coordenadas Paralelas mapeia os atributos em eixos paralelos igualmente espaçados. Cada elemento dos dados é exibido como uma polilinha, como ilustrado na Figura 2.2. Várias análises podem ser feitas observando as coordenadas paralelas da figura. Ao observar os atributos Att-6 e Att-9 no gráfico com linhas opacas não fica clara a concentração dos pontos em uma faixa restrita de valores, a qual fica visível na imagem com transparência. Quase todos os elementos do conjunto de dados possuem o mesmo valor para os atributos Att-0, Att-1 e Att-2, já que é visível apenas uma linha de cor mais escura no topo desses eixos.

A implementação da referida técnica, neste trabalho, foi uma adaptação de um módulo já disponível na plataforma de visualização do grupo VICG ${ }^{1}$ (Visualização, Imagens e Computação Gráfica), a VisPipeline.

Um problema que permeia quase todas as técnicas de visualização é a escalabilidade, ou seja, à medida que o número de elementos processados aumenta, o espaço visual disponível torna-se insuficiente para sua adequada representação. Todavia, a aplicação da transparência sobre as linhas pode atenuar este problema até certo ponto, como é o caso das imagens na Figura 2.2. Um segundo problema é a disposição dos eixos, pois uma determinada configuração pode favorecer ou inibir a percepção das correlações entre os atributos (Telea, 2008).

\footnotetext{
${ }^{1}$ www.vicg.icmc.usp.br
} 


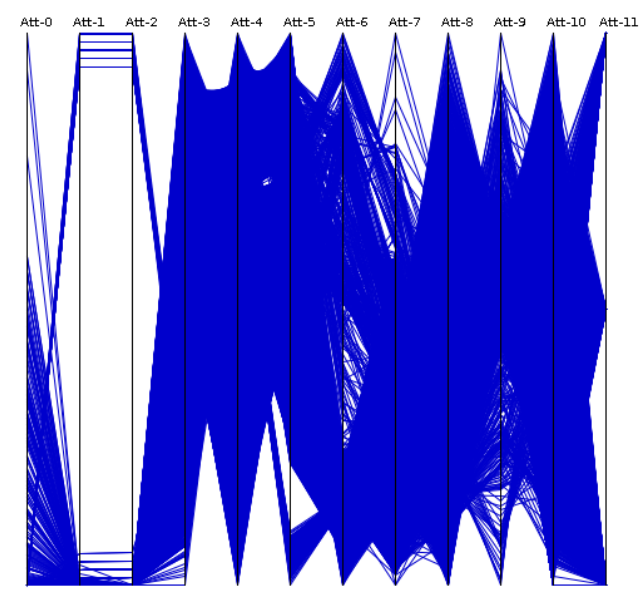

(a) Coordenadas Paralelas com linhas opacas.

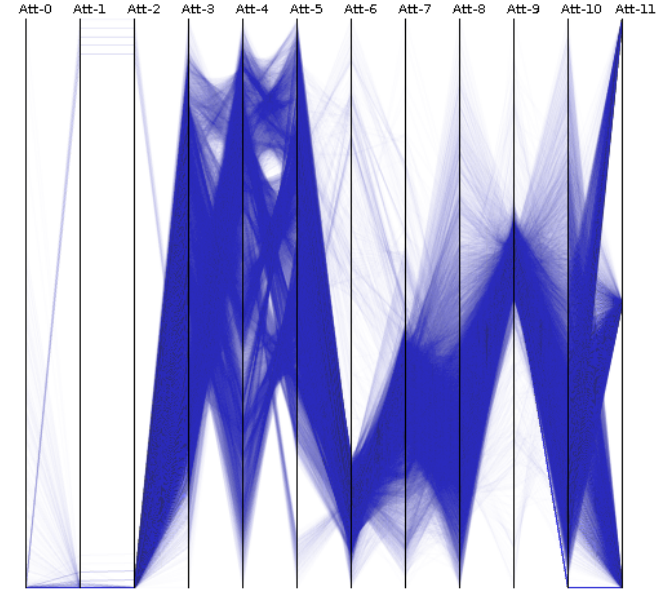

(b) Coordenadas Paralelas com linhas transparentes.

Figura 2.2: Exemplo do uso das coordenadas paralelas com um conjunto de dados de 31.703 elementos e 12 atributos.

\subsection{Técnicas de projeção multidimensional}

Segundo (Paulovich et al., 2010), as projeções multidimensionais tem recebido uma atenção significativa devido à sua habilidade de construir representações gráficas que transmitem a similaridade entre as instâncias dos dados. Ademais, as projeções apresentam a vantagem de serem eficazes para revelar os relacionamentos entre os dados, considerando simultaneamente todo o espaço de atributos, ao invés de um pequeno sub-conjunto deles. Tais habilidades nos motivam a utilizar as projeções multidimensionais para investigar os volumes multivalorados resultantes de simulações. Podemos definir formalmente uma projeção multidimensional como:

Definição 2.4.1 (Projeção Multidimensional) Seja o conjunto de dados $D \subset \mathbb{R}^{d}$ e $Y \subset$ $\mathbb{R}^{w}$, com $d>3$ e $w=\{1,2,3\}$. Então uma técnica de projeção multidimensional utiliza uma função $f: D \rightarrow Y$ que minimiza a diferença $\left|s\left(p_{i}, p_{j}\right)-\hat{s}\left(f\left(p_{i}\right), f\left(p_{j}\right)\right)\right|, \forall p_{i}, p_{j} \in D$.

Estamos especialmente interessados nas técnicas de projeção multidimensional capazes de lidar com grandes conjuntos de dados, como a Fastmap, PLMP, LSP e LAMP, introduzidas a seguir. Porém, muitas vezes precisamos utilizar uma técnica de projeção altamente precisa e não necessariamente veloz, como a PCA, Force Scheme ou a Sammon's Mapping, também introduzidas nesta seção.

\subsubsection{Análise de componentes principais (PCA)}

Análise de Componentes Principais (PCA - também conhecida como transformada Karhunen-Loeve) (Smith, 2002) gera $w$ vetores ortogonais (em nosso caso, $w=\{2,3\}$ ) que melhor representam os dados. PCA gera os vetores ortogonais pela combinação dos atributos do conjunto de dados 
e objetivando preservar a sua variabilidade. Segundo Han e Kamber (2006), PCA muitas vezes revela relações que não foram previamente suspeitas e, portanto, permite interpretações que não seriam normalmente alcançadas.

O PCA no caso bidimensional pode ser entendido pela geração dos dois eixos ortogonais, sendo o primeiro a combinação linear dos atributos que tem variância máxima no conjunto de dados, e o segundo a combinação linear dos atributos de variância máxima não correlacionada com a primeira combinação linear (Hand et al., 2001).

Para calcular o PCA, geramos a matriz $X_{n \times d}$ que corresponde à matriz de médias centradas, ou seja, subtraímos de cada elemento do conjunto de dados $D_{n \times d}$ a média do seu correspondente atributo. A matriz de covariância do conjunto de dados $D_{n \times d}$ é definida como:

$$
\Sigma_{d \times d}=X^{T} X
$$

A matriz de covariância pode ser reescrita em função dos seus autovetores, da seguinte forma:

$$
\Sigma=\mathbf{U} \Lambda \mathbf{U}^{\mathbf{T}}
$$

onde $\Lambda=\operatorname{diag}\left(\lambda_{1}, \lambda 2, \ldots, \lambda_{d}\right)$ é a matriz de autovalores ordenada decrescentemente, e $\mathbf{U}$ é a matriz ortogonal $(d \times d)$ contendo os autovetores.

Utilizando-se a matriz de autovalores $\mathbf{U}$, pode-se chegar aos componentes principais de $a$, da seguinte forma:

$$
\mathbf{a}=\mathbf{D} *\left[\mathbf{u}_{1}, \mathbf{u}_{2}, \ldots, \mathbf{u}_{\mathbf{w}}\right]
$$

onde $u_{i}$ corresponde às colunas da matriz $\mathbf{U}$ e $w$ é o número de dimensões desejado.

PCA é uma das técnicas de projeção mais largamente utilizadas, sendo sua complexidade computacional $O(w n d)$.

\subsubsection{Fastmap}

A lógica por trás da Fastmap é projetar cada ponto do conjunto de dados recursivamente em uma linha. Para tanto, dois pontos são selecionados, os quais serão conhecidos como pivôs $\left(P_{a}\right.$ e $\left.P_{b}\right)$. A linha formada pelos pivôs no espaço $d$-multidimensional é usada para projetar os demais elementos (Faloutsos e Lin, 1995), conforme ilustrado na Figura 2.3(a). O cálculo para localizar a posição $x_{i}$ do elemento $p_{i}$ é feito usando a lei do cosseno:

$$
x_{i}=\frac{s\left(P_{a}, p_{i}\right)^{2}+s\left(P_{a}, P_{b}\right)^{2}-s\left(P_{b}, p_{i}\right)^{2}}{2 s\left(P_{a}, P_{b}\right)}
$$

O processo de seleção dos pivôs consiste em localizar os pontos mais afastados do conjunto, assim é possível projetar todos os elementos entre esses pontos extremos. O processo unidimensional pode ser estendido para o espaço $m$-dimensional (no nosso caso $m=\{2,3\}$, o espaço 
visual). Para tanto aplica-se o mesmo processo recursivamente, porém, gera-se um hiper-plano $m$-dimensional perpendicular ao eixo $E=\left(P_{a}, P_{b}\right)$. A Figura 2.3(b) ilustra esse processo, em que os dois elementos $\left(P_{i}\right.$ e $\left.P_{j}\right)$ serão projetados nos pontos $P_{i}^{\prime}$ e $P_{j}^{\prime}$.

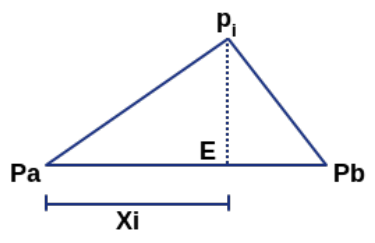

(a) Cálculo da projeção unidimensional para o elemento $P_{i}$ entre os pivôs $\left(P_{a}\right.$ e $\left.P_{b}\right)$.

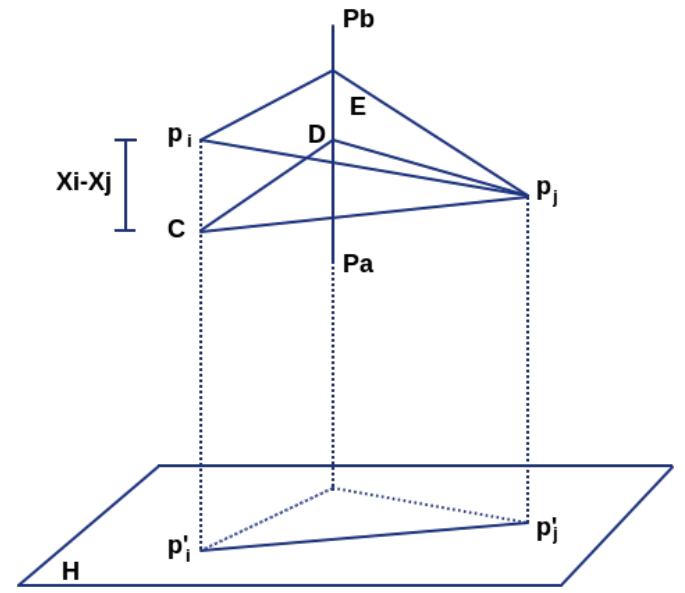

(b) Extensão da Fastmap para o caso $t$-dimensional, sendo $H$ o um hiper-plano pertendicular ao eixo $E=\left(P_{a}, P_{b}\right)$.

Figura 2.3: Ilustração da técnica Fastmap. Fonte: (Faloutsos e Lin, 1995)

Essa técnica é uma das mais rápidas. Sua complexidade computacional é determinada essencialmente pela localização dos pivôs, que pode ser feita em $O(n)$. Sua complexidade final é $O(w n)$, sendo que em nosso caso $w=\{2,3\}$, constituindo-se em uma técnica linear.

\subsubsection{Force scheme (FS)}

A técnica de projeção Force Scheme (Tejada et al., 2003) é uma adaptação da clássica estratégia Force-Directed Placement (FDP) (Paulovich e Minghim, 2008), que consiste em posicionar os elementos no espaço pela interação das forças de atração e repulsão entre eles. Tal modelo é conhecido como Modelo baseado em Molas (MM) (Paulovich e Minghim, 2008). As técnicas FDP são conhecidas pela sua alta precisão, porém elevado custo computacional, geralmente $O\left(n^{3}\right)$.

Objetivando a redução do custo computacional, em vez de calcular as forças de atração e de repulsão em um sistema de MM, o FS considera as distâncias entre os pontos do conjunto de dados. Um posicionamento aleatório é gerado inicialmente (ou utiliza-se o resultado de um método qualquer de projeção), o qual é iterativamente modificado para separar os pontos projetados muito próximo e aproximar aqueles colocados afastados, de acordo com uma medida de erro dada pela diferença da distância entre o posicionamento atual e o posicionamento ideal.

A cada iteração, FS toma $p_{i} \in D$ e calcula o vetor $\overrightarrow{v_{i j}}=\left(p_{j}-p_{i}\right), \forall p_{j} \neq y_{i} \in Y$. Então $y_{i}$ é deslocado na direção de $\overrightarrow{v_{i j}}$ por uma quantidade dada pela diferença entre a distância atual e a ideal, calculada a partir dos pontos do conjunto de dados. 
A complexidade depende de $r$ (o número de iterações) e da complexidade de cada iteração, que é $O\left(n^{2}\right)$, portanto o custo final é $O\left(r n^{2}\right)$. Embora a FS seja uma técnica de alta precisão, seu custo ainda é elevado para grandes conjuntos de dados.

\subsubsection{Sammon's mapping (SM)}

A Sammon's Mapping (Sammon, 1969) é uma técnica de projeção multidimensional não linear baseada na seguinte premissa: tentar aproximar as distâncias do espaço multidimensional para o espaço visual.

O algoritmo do SM trabalha por iterações e inicia gerando, de forma aleatória, o conjunto dos elementos projetados $Y$. Uma alternativa para a criação do conjunto $Y$ inicial é a aplicação de alguma outra técnica de projeção. Em cada iteração, a técnica calcula as distâncias entre todos os pares de elementos do conjunto no espaço projetado, objetivando conhecer se a atual configuração do conjunto $Y$ foi capaz de preservar as distâncias do espaço multidimensional, por meio da equação de erro 2.6 .

$$
E_{s m}\left(p_{i}, p_{j}\right)=\frac{1}{\sum_{i<j}\left[s\left(p_{i}, p_{j}\right)\right]} \sum_{i<j}^{n} \frac{\left[s\left(p_{i}, p_{j}\right)-\hat{s}\left(y_{i}, y_{j}\right)\right]^{2}}{s\left(p_{i}, p_{j}\right)}
$$

Caso o valor do erro $E_{s m}\left(p_{i}, p_{j}\right)$ fique acima de um certo limiar pré-estabelecido, então muda-se a configuração dos valores no espaço visual $\left(y_{i}, y_{j}\right)$ procurando diminuir o erro. A referida técnica apresenta uma alta complexidade temporal $O\left(n^{2}\right)$.

\subsubsection{Part-linear multidimensional projection (PLMP)}

Ao contrário da maioria dos métodos de projeção multidimensional, que requer a informação de distância entre todos os pares de pontos, a priori, a PLMP abre mão dessa informação em prol da eficiência computacional. A projeção é construída por meio de um mapeamento linear das coordenadas cartesianas de um subconjunto de elementos representativos, os quais são, $a$ priori, posicionados no espaço visual. O posicionamento dos elementos representativos é feito por uma técnica não linear e, apenas nesta pequena porção dos dados, faz-se necessário o cálculo da distância entre os elementos.

A qualidade da projeção final está diretamente relacionada à precisão do mapeamento dos elementos representativos. Utiliza-se para isso um método de projeção multidimensional de alta precisão, como o Force Scheme (Tejada et al., 2003). A premissa básica da PLMP é que o cômputo de uma transformação linear é uma boa aproximação do mapeamento não linear usado para projetar os elementos representativos.

Suponha que o subconjunto $D^{\prime}=\left\{p_{1}^{\prime}, p_{2}^{\prime}, \ldots, p_{t}^{\prime}\right\}, D^{\prime} \in D$ com $t<<n$, já esteja mapeado para o espaço visual $\mathbb{R}^{\{2,3\}}$. Denotando a projeção de $p_{i}^{\prime}$ em $\mathbb{R}^{\{2,3\}}$ por $\bar{p}_{i}^{\prime}$, o mapeamento linear ideal $\Phi$ deve satisfazer: 


$$
\Phi\left(p_{i}\right)=\bar{p}_{i}^{\prime}
$$

Para cada $i=1, \ldots, t$, a equação 2.7 permite calcular uma aproximação da transformação $\Phi$ por meio do produto entre cada linha da matriz representada por $\Phi$ e as instâncias em $D^{\prime}$. Mais especificamente, é possível gerar um sistema de equações lineares por meio da equação 2.7.

Segundo (Paulovich et al., 2010), o maior custo computacional da PLMP está relacionado ao posicionamento do grupo representativo, que pode ser feito com qualquer técnica de alta precisão, como a Force Scheme.

O principal problema da aplicação da PLMP diz respeito à seleção dos pontos representativos (amostragem). Eles irão nortear a transformação nos elementos. Portanto, uma boa projeção só é alcançada com uma boa estratégia para seleção dos elementos representativos. Entretanto, por questões de eficiência, a PLMP parte de uma amostragem aleatória e, consequentemente, não há garantias de que a projeção final será de boa qualidade. Para contornar esse problema, no nosso arcabouço nós geramos milhares de amostragens e as projetamos, em uma fase de pré-processamento. Em seguida, selecionamos as melhores com base no valor de stress e em uma avaliação visual. Dessa forma, possivelmente encontraremos uma amostragem satisfatória.

\subsubsection{Least square projection (LSP)}

A Least Square Projection é baseada na hipótese de que cada elemento $p_{i}$ do conjunto de dados $D$ pode ser representado como uma combinação convexa dos seus vizinhos mais próximos, seja $N_{i}=p_{i_{1}}, \ldots, p_{i_{k_{i}}}$ o conjunto com $k_{i}$ vizinhos mais próximos de $p_{i}$. Sendo as coordenadas $\left(x_{p_{i_{j}}}, y_{p_{i_{j}}}\right)$ de cada elemento $p_{i_{j}} \in N_{i}$ quando mapeado para $\mathbb{R}^{2}$. Dessa forma, a combinação convexa das coordenadas bidimensionais de $p_{i}$ pode ser escrita como:

$$
\left(x_{p_{i}}, y_{p_{i}}\right)=\sum_{p_{i_{j}} \in N_{i}} \alpha_{i j}\left(x_{p_{i_{j}}}, y_{p_{i_{j}}}\right)
$$

onde $\alpha_{i j}>0$ e $\sum \alpha_{i j}=1$. Quando $\alpha_{i j}=\frac{1}{k_{i}}$, tem-se que $p_{i}$ é o centróide de $N_{i}$.

Para cada elemento em $D$ da equação 2.8 , pode-se montar dois sistemas lineares homogêneos:

$$
L \mathbf{x}=0 ; L \mathbf{y}=0
$$

onde $\mathbf{x}$ e $\mathbf{y}$ são vetores representando as coordenadas $x$ e $y$ do elemento mapeado e $L$ a matriz derivada da equação 2.8 .

O sistema de equações lineares 2.9 não possui informação geométrica, então a LSP adiciona pontos de controles de forma a suprir essa lacuna. Os pontos de controle são um subconjunto dos dados escolhido cuidadosamente para representar todo o conjunto.

A melhor escolha para os pontos de controle seriam instâncias que fossem os centróides de cada subgrupo $N_{i}$ (sendo $1 \leq i \leq n_{c}=\sqrt{n}$ ), por isso é utilizado o método $k$-medoides para 
localizar os pontos de controle. Uma vez selecionado o grupo de controle, suas coordenadas cartesianas são calculadas com uma técnica altamente precisa como a Force-Directed Placement (FDP) (Paulovich e Minghim, 2008).

Segundo Paulovich et al. (2009), a LSP possui dois grandes gargalos: o primeiro é o cálculo dos vizinhos mais próximos, que é de longe o que possui maior custo computacional; o segundo é a resolução do sistema linear, que pode consumir muito recurso de memória, como também tempo de processamento. Esses gargalos levam a complexidade da técnica a ser $O\left(n^{\frac{3}{2}}\right)$.

\subsubsection{Local affine multidimensional projection (LAMP)}

LAMP (Joia et al., 2011), assim como os métodos locais (PLMP e LSP), faz uso de um subconjunto de pontos chamados de pontos de controle e sua projeção no espaço visual. $\mathrm{O}$ usuário pode manipular os pontos de controle no espaço visual, e essa modificação é considerada na construção do sistema final. Para cada ponto a ser projetado, é construído um mapeamento ortogonal afim que projeta os dados no espaço visual, sendo que o mapeamento segue o layout definido pelos pontos de controle.

Dado um elemento $p \in D$, a LAMP projeta o referido ponto encontrando a melhor transformação afim $f(p)=p M+t$ que minimiza a expressão 2.10 .

$$
\sum_{i} \alpha_{i}\left\|f_{x}\left(p_{i}\right)-y_{i}\right\|^{2}
$$

restringindo $M^{T} M=I$, com $I, t, M$ e $\alpha_{i}$ sendo, respectivamente, a matriz identidade, o vetor de translação, a matriz de transformação linear e os pesos escalares definidos na equação 2.11.

$$
\alpha_{i}=\frac{1}{p_{i}-p}
$$

Ao se restringir $M^{T} M=I$, garante-se que a transformação afim irá se comportar como uma transformação de corpo rígido, ou seja, os dados só podem ser rotacionados e/ou transladados, evitando os efeitos de escala e de cisalhamento, exatamente o que é preciso para preservar as distâncias no processo de projeção multidimensional. A técnica pode ser executada em $O(k n)$ (com $k$ operações), o que a torna um método linear.

\subsection{Avaliando a qualidade das projeções}

Dentre as formas de avaliar a qualidade de uma projeção, destacam-se três técnicas: função de estresse (stress function), curva de estresse (stress curve) e a preservação da vizinhança (neighborhood preservation).

A função de estresse mais largamente utilizada na literatura (Faloutsos e Lin, 1995; Paulovich et al., 2010; Joia et al., 2011) foi definida por Kruskal (Kruskal, 1964). Essa função tenta 
quantificar a perda de informação ocorrida no mapeamento da projeção. Seu cômputo é dado pela equação 2.12 .

$$
\text { Kruskal }=\sqrt{\frac{\sum_{i<j}\left(\hat{s}\left(p_{i}, p_{j}\right)-s\left(p_{i}, p_{f}\right)\right)^{2}}{\sum_{i<j} \hat{s}\left(p_{i}, p_{j}\right)}}
$$

Segundo Paulovich e Minghim (2008), embora a função de estresse seja capaz de mensurar a quantidade de informação perdida, ela não é necessariamente uma medida confiável com relação a identificação de estruturas de interesse, como agrupamentos e tendências. Uma alternativa inspirada no gráfico de dispersão é a curva de estresse formada pelo gráfico de dispersão das distâncias originais pelas distâncias projetadas $s\left(p_{i}, p_{j}\right) \times \hat{s}\left(y_{i}, y_{j}\right)$ (Joia et al., 2011). Uma ilustração de tal gráfico pode ser vista na Figura 2.4. Uma projeção ideal, capaz de preservar $100 \%$ das distâncias, produziria uma curva com todos os pontos ao longo da diagonal principal do gráfico.

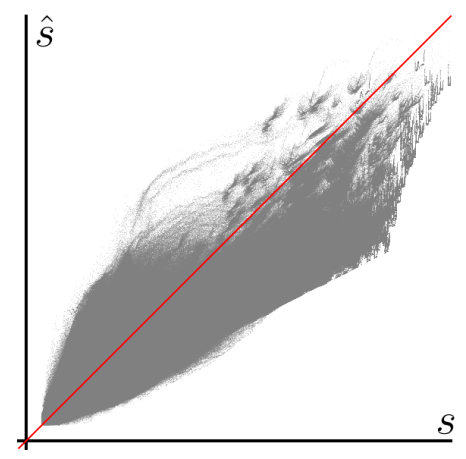

Figura 2.4: Ilustração da curva de estresse.

O gráfico da preservação da vizinhança mostra o percentual de pontos vizinhos no espaço original que ainda permanecem vizinhos no espaço projetado, considerando um intervalo de número de vizinhos. É calculado o percentual para cada ponto. Um valor igual a 1.0 indica $100 \%$ de preservação dos vizinhos. Geralmente, estamos interessados em projeções capazes de preservar ao máximo a relação de vizinhança. Na Figura 2.5 tem-se um exemplo do gráfico da preservação da vizinhança.

\subsection{Técnicas de agrupamento}

Espera-se que a projeção gere visualizações em que os elementos altamente similares apareçam como agrupamentos perceptíveis pelo usuário. Entretanto, nos conjuntos de dados utilizados neste trabalho, as projeções nem sempre revelam de forma evidente os grupos. Isso se deve, em parte, ao elevado número de elementos dos conjuntos, como também a não separação dos dados, já que fenômenos reais nem sempre apresentam grupos inconfundíveis. Contudo, técnicas de agrupamento podem ser aplicadas de forma a auxiliar a identificação dos elementos similares e a exploração dos dados. 


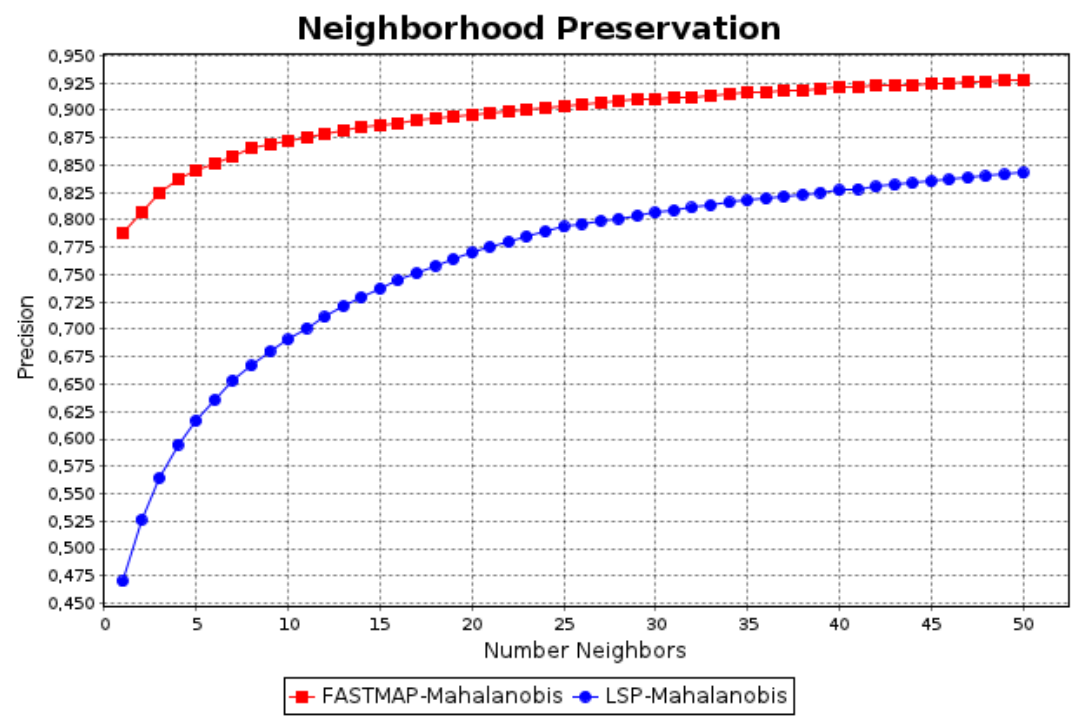

Figura 2.5: Ilustração do gráfico da preservação da vizinhaça. Observa-se que a técnica representada pela linha vermelha foi capaz de preservar melhor a vizinhaça.

Nosso critério para a seleção das técnicas de agrupamento é baseado na simplicidade e na facilidade de implementação. Ademais centramos nossa escolha em técnicas que particionam o conjunto de dados. A seguir, apresentam-se alguns métodos de agrupamento, como também uma medida para avaliar a qualidade dos grupos gerados.

Um método de agrupamento deve produzir $k$ subgrupos $G$, ou seja, $G=\left\{G_{1}, G_{2}, \ldots, G_{k}\right\}$, de tal forma que $D=\left\{G_{1} \cup G_{2} \cup \ldots \cup G_{k}\right\}$, em que $\left\{G_{1} \cap G_{2} \cap \ldots \cap G_{k}\right\}=\emptyset$. Define-se como centro de massa do subgrupo $G_{i}$ o valor médio de todos os elementos pertencente ao subgrupo. Denomina-se como centroide $\left(m_{i}\right)$ do subgrupo $G_{i}$ o centro de massa do subgrupo, sendo $M=\left\{m_{1}, m_{2}, \ldots, m_{k}\right\}$ o conjunto de centroides dos subgrupos $G=\left\{G_{1}, G_{2}, \ldots, G_{k}\right\}$. Denomina-se medoide $\left(m e_{i}\right)$ do subgrupo $G_{i}$, a instância $p \in G_{i}$ que possui o valor mais próximo ao centro de massa do subgrupo.

\subsubsection{K-Means e variações}

O k-means é uma técnica de agrupamento computacionalmente fácil, rápida e de baixa necessidade de memória (Mirkin, 2005). O k-means agrupa o conjunto de dados $D$ em $k$ subgrupos, sendo o valor de $k$ fornecido pelo usuário. O algoritmo mantém uma listagem dos $k$ subgrupos e seus centroides e procede em iterações. Antes da primeira iteração, a lista de centroides é iniciada aleatoriamente. O algoritmo finaliza quando a localização dos centroides não mais se alterar durante uma iteração. A cada iteração são executados os seguintes passos (Pelleg e Moore, 2000):

1. Para cada ponto $p \in D$, encontre o centroide $m_{i}$ mais próximo de $p$. Associe $p$ ao subgrupo $G_{i}$ ao qual o centroide $m_{i}$ está vinculado. 
2. Recalcule a nova posição dos centroides $M$, fazendo, para cada subgrupo $G_{i}$, o valor do centro de massa.

3. Caso não haja mudança na lista dos centroides, então retorne os $k$ subgrupos $G$ associados à lista de centroides $M$. Do contrário, retorne ao passo 1 .

O algoritmo atualiza os subgrupos de acordo com a chamada regra da distância mínima (minimum distance rule), que atribui a cada instância $p \in D$ o subgrupo que está mais próximo a ela.

O k-means minimiza a função conhecida como critério do erro quadrado (square-error criterion), definida como:

$$
E=\sum_{i=1}^{k} \sum_{p \in G_{i}}\left|p-m_{i}\right|^{2},
$$

sendo $E$ a soma do erro quadrático para todos os pontos no conjunto de dados $D$, sendo $p$ um ponto do subgrupo $G_{i}$ e $m_{i}$ o centroide que representa $G_{i}$.

A complexidade computacional do $k$-means é medida em função de quatro variáveis: o número de pontos $(n)$ no conjunto de dados $(D)$, a dimensionalidade $(d)$, o número de agrupamentos $(k)$ e o número de iterações $(i t)$. Portanto, a complexidade final será $O(n * k * d * i t)$. Para conjuntos de dados muito grandes, no qual $n>>d$, o valor de $k * d * i t$ acaba se transformando em um coeficiente $c$, então o custo computacional fica em $O(c * n)$.

Um dos principais problemas do $k$-means diz respeito à escolha dos centroides iniciais, já que uma má seleção desses valores iniciais pode afetar a velocidade de convergência do algoritmo, como também o resultado final (Mirkin, 2005).

A necessidade do usuário definir $k$ pode ser vista como uma desvantagem. Além disso, o k-means não é adequado para descobrir agrupamentos de formato não-convexo. Tipicamente o algoritmo localiza subgrupos de forma esférica. O k-means é sensível a ruído e a outliers, que podem influenciar substancialmente no valor dos centroides (devido ao erro quadrático) (Han e Kamber, 2006).

O bisecting $\boldsymbol{k}$-means é uma variante que trabalha tanto de forma divisória como de forma hierárquica, produzindo agrupamentos por meio de sucessivas aplicações do $k$-means (Kashef e Kamel, 2009). A ideia por trás do bisecting k-means para a obtenção dos $k$ agrupamentos é simples. O algoritmo mantém uma listagem dos subgrupos e dos centroides, porém inicia apenas com um único subgrupo que corresponde a todos os pontos do conjunto de dados, e procede dividindo o maior subgrupo utilizando o $k$-means até atingir o número $k$ de agrupamentos.

Segundo Mirkin (2005), uma das vantagens do bisecting k-means é que ele é menos suscetível aos problemas de inicialização encontrados no $k$-means. Uma segunda vantagem, que pode ser bastante desejável, é que ele tende a gerar agrupamentos de tamanho semelhante.

Um dos problemas é que, enquanto que o k-means garantidamente localiza agrupamentos que representam o mínimo local com relação ao erro quadrático, o bisecting k-means utiliza o 
k-means localmente para dividir os agrupamentos. Portanto, o conjunto final de subgrupos não representa agrupamentos com o mínimo local com relação ao erro quadrático (equação 2.13) (Mirkin, 2005). Ademais, o bisecting k-means sofre da mesma sensibilidade a ruído e a outliers.

O $\boldsymbol{k}$-medoids foi concebido com o propósito de diminuir a sensibilidade ao ruído e aos outliers. Para tanto, ao invés de tomar como referência o centro de massa do agrupamento, o algoritmo k-medoids escolhe uma instância do agrupamento como ponto de referência. Cada instância restante é agrupada com relação aos pontos de referência selecionados para os $k$ subgrupos. Dessa forma, o algoritmo obtém os agrupamentos baseado na minimização da soma das dissimilaridades entre os elementos e seus respectivos pontos de referência. Portanto, o critério do erro absoluto (absolute-error criterion) é definido da seguinte forma:

$$
E_{a}=\sum_{j=1}^{k} \sum_{p \in G_{j}}\left|p-o_{j}\right|,
$$

sendo $E_{a}$ a soma do erro absoluto para todas as instâncias em $D$, sendo $p$ um ponto do subgrupo $G_{j}$, e $o_{j}$ o ponto de referência associado a $G_{j}$. Em geral, o algoritmo itera até que, eventualmente, cada ponto de referência se torne o medoide do subgrupo ao qual pertence.

A complexidade do $k$-medoids é $O\left(k(n-k)^{2}\right)$, o que reflete um custo quadrático, inapropriado para lidar com grandes conjuntos de dados. Uma segunda desvantagem é a necessidade de fornecer o número de agrupamentos $k$, desvantagem essa compartilhada por todas as variações do $k$-means. Sua principal vantagem, conforme explicado anteriormente, é a maior robustez ao lidar com dados com ruído e com outliers (Tan et al., 2005).

\subsubsection{X-Means e variações}

Pelleg e Moore (2000) propuseram uma extensão do k-means que é capaz de estimar, de forma eficiente, o número de agrupamentos $k$ por meio da otimização do critério de informação bayesiano (Bayesian Information Criterion - BIC).

No $x$-means a BIC é usada globalmente e localmente objetivando identificar o melhor número de agrupamentos, como também selecionar os subgrupos a serem divididos, respectivamente. Seja o conjunto $D=\left\{p_{1}, p_{2}, \ldots, p_{n}\right\}$ contendo $n$ pontos no espaço $d$-dimensional, e seja uma

família de modelos alternativos $M_{j}=\left\{M_{k_{\text {min }}}, M_{k_{\text {min }+1}}, \ldots, M_{k_{m a x}}\right\}$ (diferentes modelos, correspondendo a soluções com diferentes valores de $k$ ). Dessa forma, utiliza-se a probabilidade posterior $P\left(M_{j} \mid D\right)$ para ranquear os diferentes modelos (Gan et al., 2007).

O algoritmo $x$-means inicia com o usuário especificando o intervalo $\left[k_{\min }, k_{\max }\right]$ no qual um razoável valor de $k$ pode ser encontrado. Dessa forma, o algoritmo atribui inicialmente $k=k_{\text {min }}$ e continua incrementando o número de centroides nas regiões onde se faz necessário, até que o valor máximo seja atingido. Durante o processo a melhor configuração de centroides com o maior valor da BIC é selecionada.

O algoritmo consiste em duas operações principais, que são repetidas até a condição de parada, conforme mostrado a seguir: 
1. Melhore-parâmetros.

2. Melhore-estrutura.

3. Se $k>k_{\max }$ pare e retorne o modelo com melhor valor. Senão, retorne ao passo 1 .

Em melhore-parâmetros a operação é simples: consiste em executar o $k$-means convencional.

Em melhore-estrutura a operação localiza se e onde novos centroides devem aparecer. Isto é possível ao subdividir alguns subgrupos em dois. Essa decisão é tomada da seguinte forma: os agrupamentos com menores valores BIC são divididos para verificar o resultado da função BIC dos agrupamentos filhos. Caso os filhos tenham maior valores que o pai, então o subgrupo é de fato dividido, do contrário permanece o agrupamento pai.

Segundo Bristow (2010), um dos problemas do $x$-means é a tendência de superestimar o verdadeiro número de $k$. Porém, o autor relata que, ainda assim o algoritmo uma boa precisão, com erro inferior a $15 \%$ e desvio padrão de $5 \%$ (valores advindos de seus experimentos).

Pelleg e Moore (2000) afirmam que o critério BIC para seleção dos agrupamentos a serem subdivididos não é o único possível, outros critérios de seleção podem fazer sentido, a depender das características desejadas e dos dados. Com isso em mente, adotamos algumas variações do $x$-means, às quais aplicamos o critério BIC apenas para selecionar o melhor modelo dentre um conjunto de soluções.

É possível utilizar o critério BIC de forma unicamente global e ranquear diversos tipos de agrupamentos, e dessa forma selecionar apenas aquele com maior probabilidade. Ou seja, pode-se gerar os agrupamentos usando diversas técnicas e selecionar o melhor resultado segundo o critério BIC, com isso gerando uma variação do x-means.

Neste trabalho foram implementadas duas variações do $x$-means: (1) a utilização do bisecting $k$-means para gerar a listagem dos agrupamentos, usando o critério BIC para seleção do melhor resultado, que no restante do texto será chamado de bisecting $\boldsymbol{x}$-means, e (2) a utilização do $k$-medoids norteada pelo critério BIC, chamado de agora em diante de $\boldsymbol{x}$-medoids.

\subsection{Avaliando a qualidade de agrupamentos}

Depois de aplicado um algoritmo de agrupamento, o usuário deseja saber se o resultado reflete, de fato, uma propriedade natural do conjunto de dados ou se ele é apenas um artefato gerado pelo algoritmo (Mirkin, 2005).

Segundo Tan et al. (2005), em classificação supervisionada, a validação do modelo resultante da classificação é parte integrante do processo, e existem medidas e procedimentos largamente aceitos, por exemplo, precisão (accuracy) e validação cruzada. Já no processo de agrupamento de dados, o mesmo não acontece, mas existem métodos voltados para verificar a qualidade dos agrupamentos. 
Por exemplo, o coeficiente de silhueta verifica o quão coesos os subgrupos são, bem como sua separação. A equação 2.15 define o valor do coeficiente.

$$
S=\sum_{i=1}^{n} \frac{b(i)-a(i)}{\max (a(i), b(i))}
$$

onde $a(i)$ é a distância média do elemento $p_{i}$ aos pontos em seu agrupamento, e $b(i)$ é a menor distância média do elemento $p_{i}$ em relação aos pontos em todos os outros agrupamentos. Os valores $a(i)$ e $b(i)$ medem coesão e separação, respectivamente.

O valor do coeficiente pode variar entre -1 e 1 . Valores negativos são indesejáveis, porque correspondem ao caso no qual $a(i)$, a distância média dos pontos no agrupamento, é maior do que $b(i)$, a menor distância média para pontos em outros agrupamentos. É desejável que o valor do coeficiente seja positivo $(a(i)<b(i))$, e que o valor de $a(i)$ fique o mais próximo possível de 0 , já que o coeficiente assume seu valor máximo 1 , quando $a(i)=0$.

\subsection{Considerações finais}

Este capítulo descreveu algumas técnicas de visualização de dados multidimensionais, como a matriz de gráfico de dispersão e as coordenadas paralelas. Dentre as técnicas que lidam com dados multidimensionais, destacaram-se as projeções multidimensionais, devido em parte, à sua habilidade em preservar as relações de similaridade, capacidade de lidar com grandes conjuntos de dados e de tratar todos os atributos dos dados. Apresentaram-se, também algumas técnicas de agrupamento, objetivando auxiliar na identificação dos elementos altamente similares no conjunto estudado. Por fim, foi discutida a validação dos grupos identificados pelas técnicas de agrupamento. 


\section{Visualização de volumes variantes no tempo}

\subsection{Considerações iniciais}

A habilidade dos cientistas em visualizar e explorar fenômenos variantes no tempo é essencial para assegurar a correta interpretação e análise dos dados, provocando entendimento e percepção, e comunicando esse insight para outros. Em particular, a visualização interativa dá aos cientistas liberdade para explorar os dados nos domínios do espaço e do tempo (Ma, 2003). Portanto, os sistemas para visualizar os volumes multivalorados devem conciliar os seguintes pré-requisitos:

- gerenciar a imensa quantidade de dados, característica dessas aplicações;

- permitir o rastreamento e a evolução das características de interesse à medida que evoluem no tempo;

- mapear as informações relevantes para as características visuais apropriadas;

- possibilitar que o usuário seja capaz de navegar entre diferentes intervalos e instantes de tempo;

- permitir que o usuário explore, de maneira simples, o volume relativo a uma fatia de tempo;

- apoiar o usuário na busca de correlações e associações nos dados entre sucessivas instâncias de tempo e

- fornecer meios para uma eficiente investigação temporal, tanto no domínio espacial como no espaço de características. 
Satisfazer tais requisitos tem se revelado uma tarefa árdua e complexa. Na literatura das duas últimas décadas é possível observar o grande esforço da comunidade científica para superar esses problemas. Neste Capítulo são apresentadas algumas soluções alcançadas pelos pesquisadores. Na Seção 3.2 tem-se trabalhos que lidam com malhas volumétricas (voxels e células). Já a Seção 3.3 apresenta os avanços obtidos na visualização de volumes de partículas.

\subsection{Visualização de malhas volumétricas}

Samtaney, Silver, Zabusky e Cao (1994) apresentaram um algoritmo de extração e rastreamento de características de valores escalares e vetoriais em dados volumétricos multivalorados variantes no tempo. Samtaney foi um dos primeiros a usar uma combinação de técnicas de visão computacional, processamento de imagens, computação gráfica e geometria computacional no processo de visualização exploratória de volumes variantes no tempo, objetivando a extração, rastreamento e visualização das características em volumes.

Doleisch, Muigg e Hauser (2004) adaptaram a ferramenta SimVis, a qual combina técnicas de visualização da informação (Info Vis) com visualização científica (SciVis) para acessar concorrentemente tanto o espaço do objeto, bem como o espaço dos atributos multidimensionais para apoiar a exploração dos volumes multivalorados. Através do linking-and-brushing em gráficos de dispersão ou em histogramas dos atributos, o usuário pode observar as regiões de interesse realçadas no espaço do objeto.

Tanto Schulze e Forsberg (2004) como Gruchalla e Marbach (2004) desenvolveram ferramentas interativas para a exploração de volumes de dados variantes no tempo. Eles adotaram como principal abordagem a imersão em um ambiente de realidade virtual. Nessas ferramentas, é possível explorar múltiplas variáveis dos volumes multivalorados "andando" no volume relativo a um instante de tempo específico, no ambiente virtual, e mapeando os atributos escalares em cores.

O trabalho de Jiang et al. (2004), lida com volumes multivalorados advindos de uma simulação de um furacão, considerando que o furacão é um tipo de vórtice. Ele contém uma região central, comumente chamada de olho do furacão. Através dessa região que os cientistas monitoram o desenvolvimento da tormenta. Jiang et. al. aplicaram quatro técnicas de detecção de vórtices (combinatorial method, normalized helicity, $\lambda 2$ method e swirl parameter) aos dados, porém elas mostraram-se incapazes de localizar o olho do furação. Eles perceberam que a velocidade próxima ao olho do furação é caracterizada pela mínima local. Como os métodos tradicionais de detecção de vórtices falharam, eles implementaram um framework que automaticamente identifica o olho do furação, procurando pelo mínimo local da velocidade, combinado com uma área de baixa pressão.

Schafhitzel e Weiskopf (2004) utilizam planos de visualização 2D advindos de recortes do volume 3D do conjunto de dados do furação, o mesmo utlizado por Jiang et al. (2004). Os campos escalares são visualizados por meio desses planos, que podem ser utilizados isoladamente ou em 
combinação com a representação do campo vetorial. Usando esse recurso, o usuário é capaz de investigar regiões especiais, por exemplo, as regiões com uma aparência elevada de vórtices, sem perder as informações do fluxo. A Figura 3.1 exibe três fatias, à esquerda mostra-se a visualização do fluido colorido pelo valor da pressão, no centro tem-se a visualização do campo vetorial e à direita apresenta-se a visualização do atributo vorticidade.

Eles concluiram que a pressão próximo ao olho do furacão é muito baixa, criando um cinturão formado por um vácuo com pressão perto de zero, e próximo ao centro do olho a pressão sobe consideravelmente. Isto pode explicar o efeito de sucção no centro (Schafhitzel e Weiskopf, 2004).
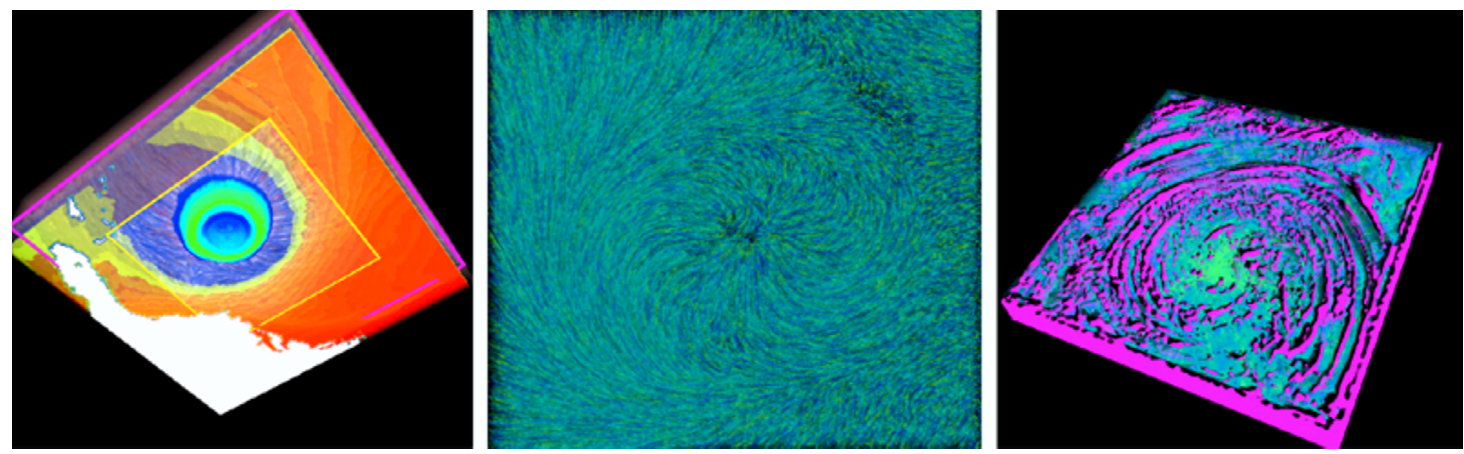

Figura 3.1: Esquerda: pressão e fluído - centro: rotação - direita: vórtices. Fonte: (Schafhitzel e Weiskopf, 2004)

Um dos trabalhos mais interessantes na área de visualização exploratória de volumes é o artigo de Tzeng \& Ma (Tzeng, 2005). Eles apresentam uma função de transferência adaptativa e inteligente que pode se adaptar às mudanças temporais dos dados. Este método permite que o usuário especifique características de interesse com as funções de transferência unidimensionais nos principais instantes de tempo do conjunto de dados, e então a função de transferência adaptativa é gerada para toda a seqüência, usando uma rede neural perceptron. A principal desvantagem dessa técnica é o tempo considerável necessário para o processo de treinamento.

A Figura 3.2 apresenta o resultado da extração e rastreamento de uma característica em um conjunto de dados de fluxo turbulento de vórtices com 100 instantes de tempo. Da esquerda para a direita, de cima para baixo, existem seis imagens geradas da aplicação da técnica no intervalo de tempo 50 até 74 . A característica rastreada é renderizada em vermelho, com o volume original a fornecer contexto. Os resultados mostram que a característica rastreada move-se e muda de forma ao longo do tempo e se divide próximo do final.

Blaas, Botha e Post (2007) apresentam uma ferramenta de visualização de dados médicos volumétricos baseada em múltiplas visões que são coordenadas entre si, sendo altamente interativa. A abordagem tem como metas auxiliar na investigação de combinações de características capazes de separar os pontos no espaço do objeto (segmentar o volume); localizar agrupamentos e padrões de relacionamentos; visualização seletiva baseada em intervalos de valores de certas características; investigação de possíveis características e sua adequação para segmentação do volume. A Figura 3.3 mostra a visão de quatro componentes da ferramenta desenvolvida: 

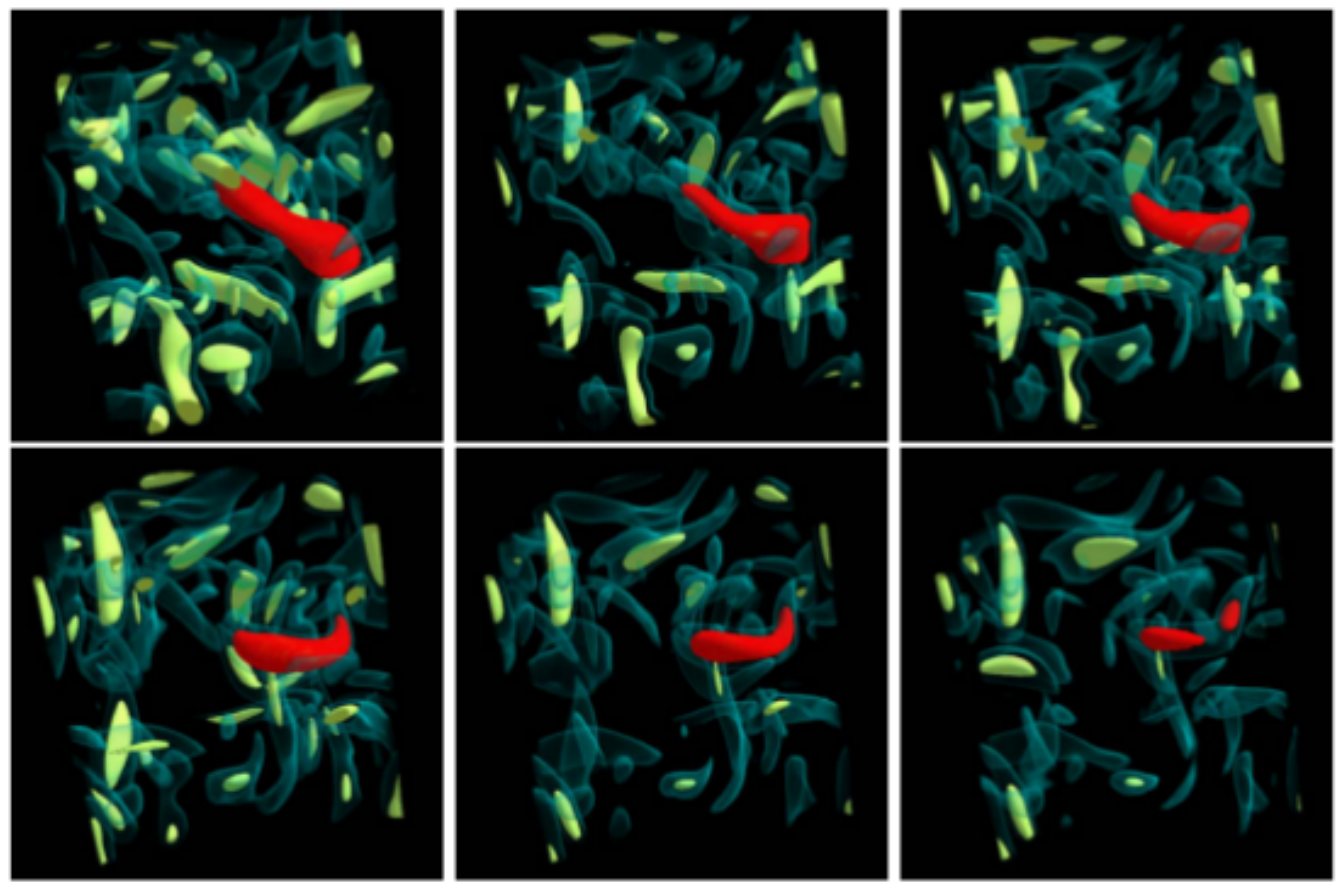

Figura 3.2: Resultado do rastreamento de uma característica. Seis instantes de tempo do intervalo entre 50 até 74 são apresentados. A característica de interesse é renderizada em vermelho. Fonte: (Tzeng, 2005).

histograma (distribuição das intensidades associadas aos voxels), gráfico de dispersão (as setas indicam a coloração), visão de fatia (slice-viewer) e visão de fatia pseudo-colorida (pseudo-color slice-viewer).
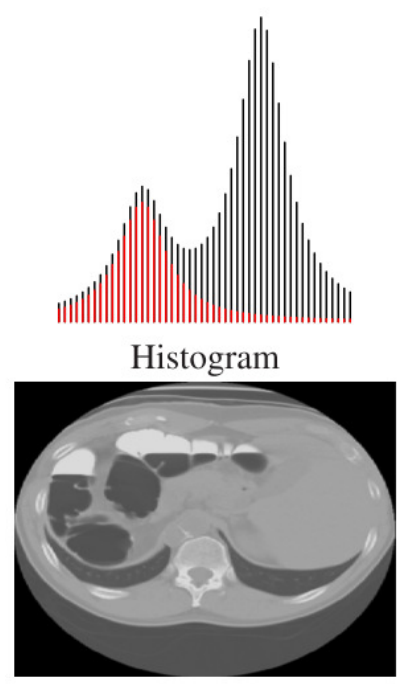

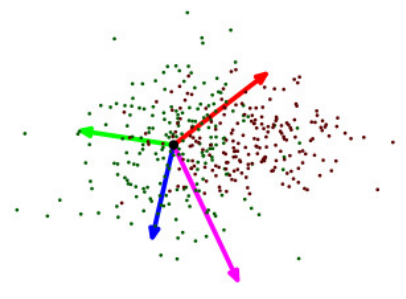

Scatterplot

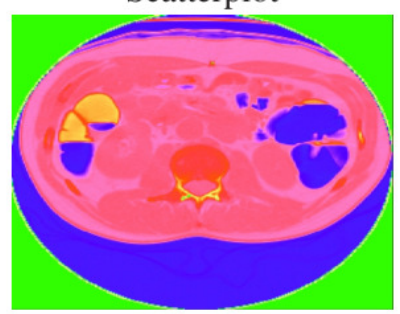

Figura 3.3: Visão de quatro componentes coordenados: histograma, gráfico de dispersão, visão de fatia e visão de fatia pseudo-colorida. Fonte: (Blaas et al., 2007) 
Linsen, Van Long, Rosenthal e Rosswog (2008) apresentaram uma abordagem baseada na extração de superfícies em volumes de dados multidimensionais. A superfície segmenta os dados com respeito a uma função multivariada definida sobre o espaço de atributos. A exploração do espaço de atributos é executada por meio de um método de agrupamento hierárquico baseado em densidade. Os agrupamentos resultantes são exibidos na forma de conjuntos de níveis de densidade em um layout produzido pela técnica 3D star coordinates (Kandogan, 2001). Um usuário pode utilizar o layout para selecionar os agrupamentos de interesse que correspondem a superfícies do volume. A Figura 3.4 apresenta as representações gráficas utilizadas nessa abordagem.

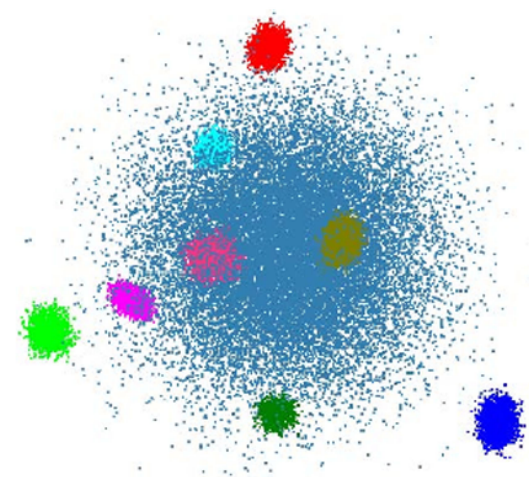

(a) Visualização dos agrupamentos no espaço de atributos: extração de oito agrupamentos e projeção em um espaço 3-D usando a técnica star coordinates.

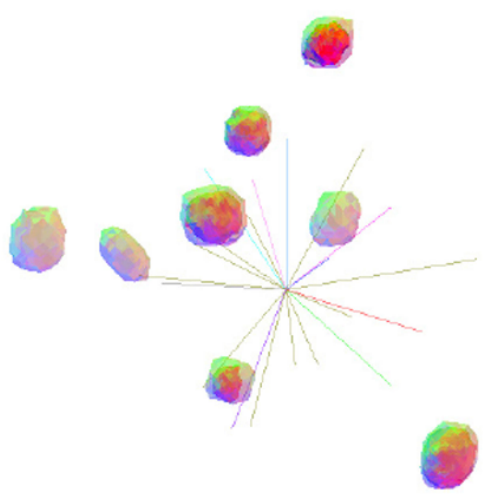

(b) Visualização dos agrupamentos por meio do rendering das superfícies.

Figura 3.4: Representações gráficas resultantes da abordagem definida por Linsen et al. (2008).

Hsu, Mei, Correa e Ma (2010) apresentam um mecanismo de extração e rastreamento de características bastante convencional, usando técnicas apresentadas por Samtaney et al., como crescimento de regiões usando como base o instante de tempo anterior. Porém, sua principal contribuição foi permitir a visualização exploratória de volumes variantes no tempo por meio de imagens geradas a partir dos volumes, diminuindo significativamente a quantidade de dados e permitindo uma interação muito mais rápida.

Daniels, Anderson, Nonato e Silva (2010) apresentam uma técnica flexível para a exploração de campos vetoriais de dados volumétricos, focando em fatias do volume, ou seja, tratam campos vetoriais $2 \mathrm{D}$. Um grafo bidirecional é construído a partir dos atributos das instâncias o qual infere as relações globais no campo vetorial. O usuário identifica um conjunto de pontos de controle a partir da vizinhança dos vetores que são representativos da característica desejada. Estes pontos são mapeados no espaço dos atributos e integrados com o grafo de atributos. Então, um sistema linear projeta os pontos do espaço de atributos para a tela de textura interativa. Pode-se atribuir cores aos pontos projetados e, dessa forma, mapear as cores no campo vetorial. A Figura 3.5 ilustra a interface da técnica de Daniels et. al. 


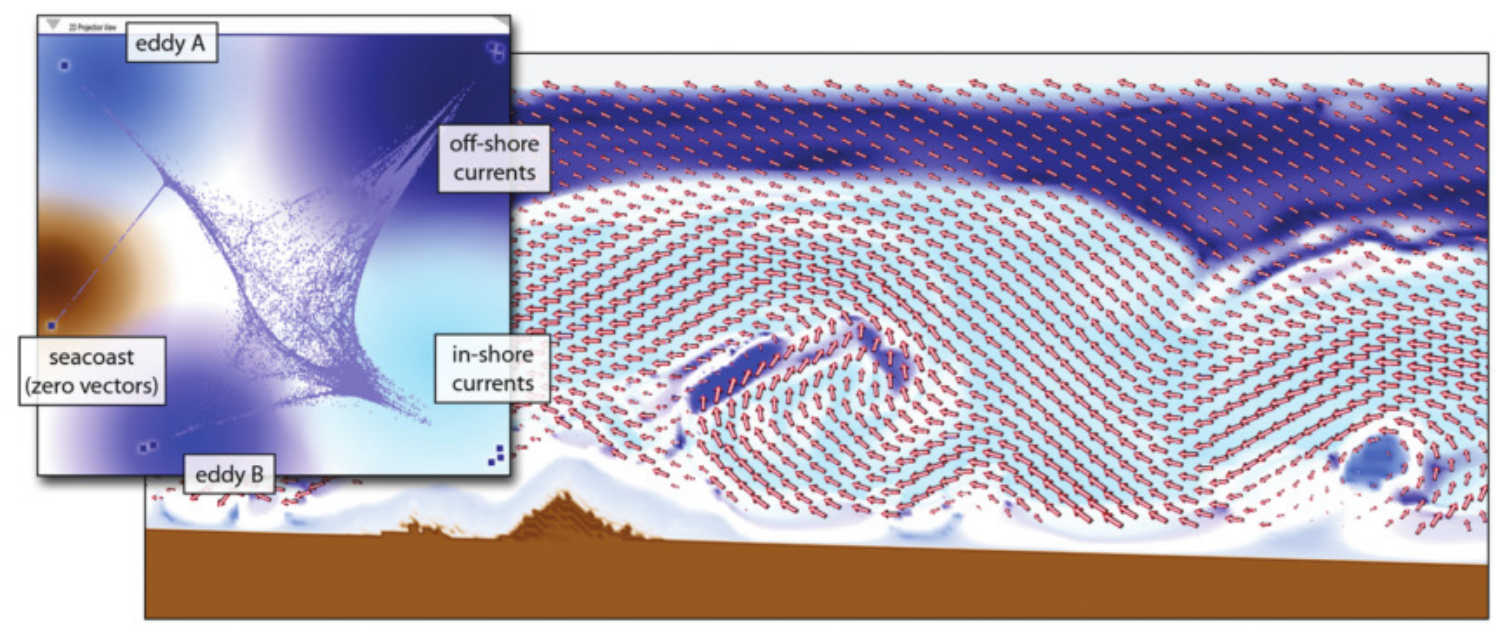

Figura 3.5: Demonstração da aplicação da técnica de Daniels et. al. sobre o conjunto de dados da simulação das correntes marítmas ao longo da costa do pacífico. No lado esquerdo a projeção do espaço de atributos colorida pelo usuário. Com as atribuições das cores no espaço de atribuitos é feito o mapeamento de textura no campo vetorial. Fonte: (Daniels et al., 2010)

Ferdosi e Roerdink (2011) tentam otimizar duas técnicas oriundas da Visualização da Informação (coordenadas paralelas e gráficos de dispersão) para lidar com grandes quantidades de dados, por exemplo, dados volumétricos. Um dos principais problemas com as coordenadas paralelas e os gráficos de dispersão é a sobreposição e desorganização à medida que a quantidade de dados aumenta, tornando a interpretação difícil. Os autores propõem um método para reordenação dos atributos das coordenadas paralelas, de tal forma que as estruturas em altas-dimensões (se houver) tornam-se mais facilmente percebidas. Já a desordem nos gráficos de dispersão é reduzida por uma filtragem, com o objetivo de melhorar a visualização.

Eles propuseram um novo método que procura por estruturas de altas dimensionalidade com o objetivo de identificar a dimensionalidade de cada agrupamento presente nos dados. Uma vez identificados os agrupamentos e suas dimensionalidades, eles reordenam a sequência de eixos na visualização por coordenadas paralelas. No caso dos gráficos de dispersão eles filtram o ruído (os dados que não pertencerem aos grupos identificados) e exibem apenas os agrupamentos. A Figura 3.6 mostra tanto as coordenadas paralelas com o ordenamento original e com a aplicação da técnica, como também a matriz de gráficos de dispersão no formato original e com a aplicação da técnica.

\subsection{Visualização de volumes de partículas}

O trabalho de Gribble et al. (2006) investiga o uso de GPUs programáveis para obter taxas interativas na visualização de dados de simulação de partículas, voltado especificamente para a visualização do espaço do objeto. Sua principal contribuição foi o desenvolvimento de um sistema capaz de renderizar eficientemente glifos (glyphs) de partículas em alta qualidade, alcançando 


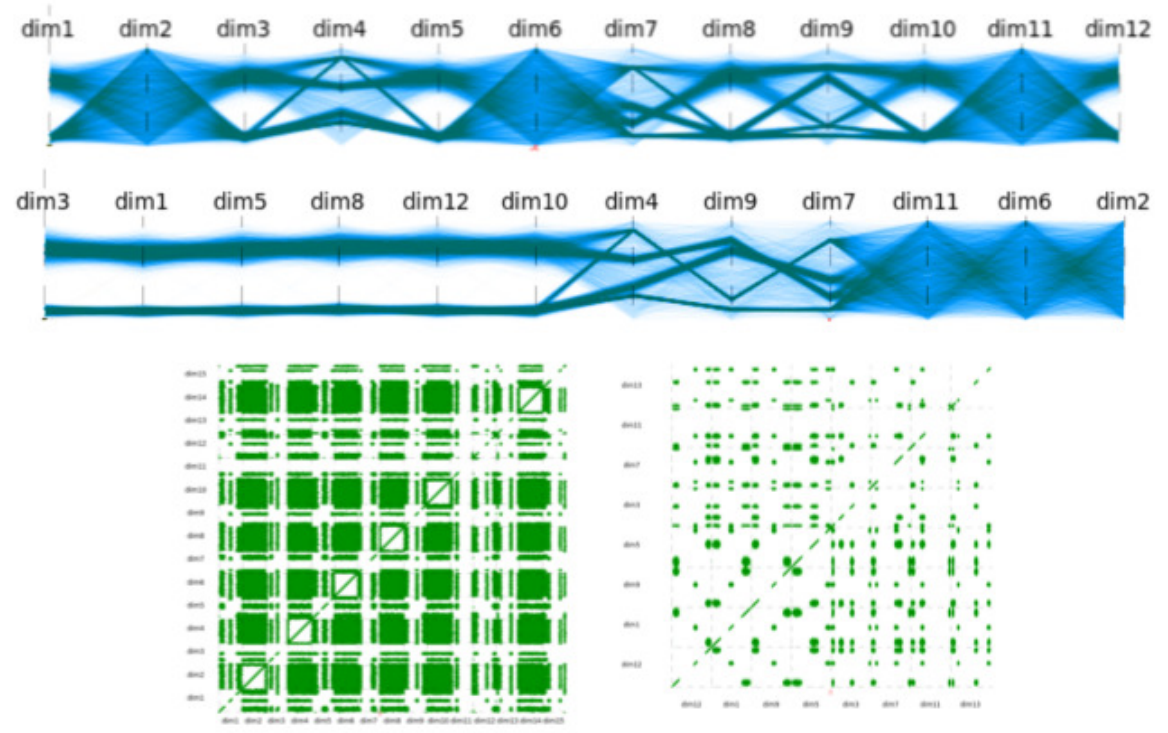

Figura 3.6: De cima para baixo: coordenadas paralelas de um conjunto de dados sintético, coordenadas paralelas reordenadas com a aplicação da técnica de Ferdosi et al. Da esquerda para direita: matriz de gráficos de dispersão sobre o mesmo conjunto de dados, matriz de gráficos de dispersão com a aplicação da técnica. Fonte: (Ferdosi e Roerdink, 2011)

desempenho interativo com hardware gráfico programável, e manipulando dados variantes no tempo interativamente. Porém, pouco foi feito para apoiar a análise temporal do espaço de atributos.

Falk e Grottel (2010), também apresentam uma abordagem voltada para a visualização do espaço do objeto. Eles desenvolveram um sistema de renderização capaz de reconstruir o volume sob demanda, minimizando o consumo de memória. A principal contribuição é um otimizado fatiador de volume baseado no ray casting (Lee et al., 2010) interligado com a reconstrução do volume sob demanda. Apesar da alta interatividade, a técnica não apresenta meios para uma análise temporal no espaço de atributos.

Já no trabalho de Co et al. (2005), os autores criaram uma ferramenta interativa e exploratória para dados de simulação de um acelerador de partículas, buscando auxiliar na percepção das relações no espaço de atributos, chamado no artigo de espaço de fase. Sua ferramenta utiliza gráficos de dispersão 2D e 3D e uma representação das partículas como um glifo 3D em um formato de disco, cuja orientação advém de um atributo vetorial (neste caso o vetor momento). Essa representação inovadora objetiva capturar a forma e a tendência das partículas afetadas por campos eletromagnéticos múltiplos. Ademais, a ferramenta desenvolvida, chamada de PPaint, é capaz de gerar animações objetivando transmitir o comportamento temporal.

Figura 3.7(a) exibe um gráfico de dispersão 3D tradicional da posição das partículas em um instante de tempo na ferramenta PPaint, já na Figura 3.7(b) é utilizada a representação em disco, em que é possível identificar o fundo do disco pela cor cinza e a frente do disco pela cor verde. Dessa forma, além dos três atributos tradicionalmente mapeados pelo gráfico de dispersão 
3D, tem-se, adicionalmente, o mapeamento de um atributo vetorial associado a orientação do disco.

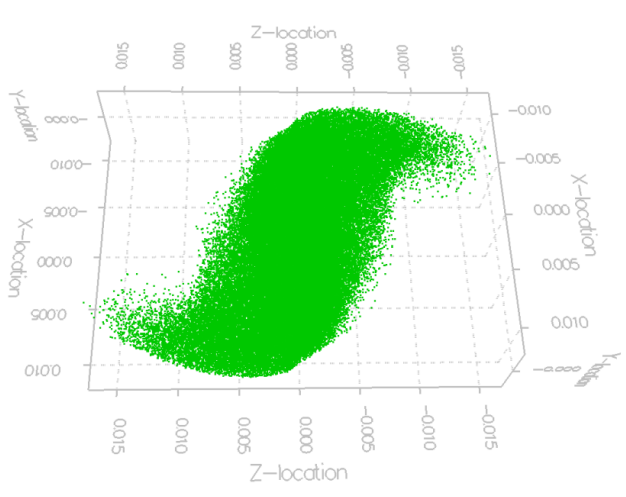

(a) Gráfico de dispersão 3D.

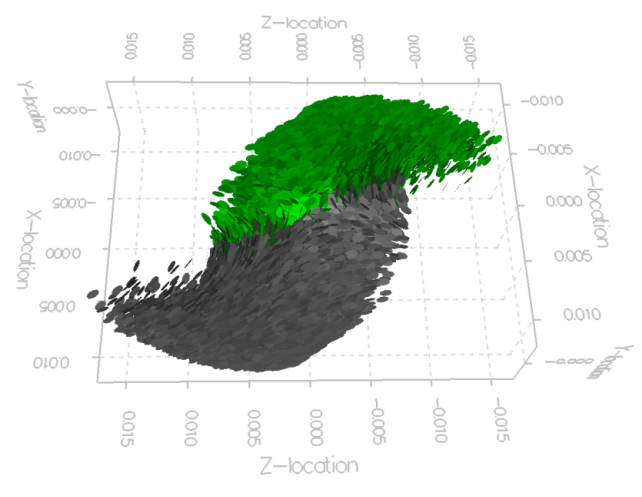

(b) Gráfico de dispersão 3D utilizando glifo em formato de disco.

Figura 3.7: Visualização de um instante de tempo da simulação de um acelerador de partículas. Fonte: (Co et al., 2005)

No trabalho de Jones, Ma, Ethier e Lee (2008) foi desenvolvido um sistema interativo e exploratório, capaz de apoiar a investigação tanto de um único instante de tempo como também de uma investigação temporal. Para a visualização do espaço do objeto, Jones et al. utilizaram duas estratégias: visualização de point sprites e a visualização da trajetória da partícula por meio de uma polilinha conhecida como path line (neste trabalho, tal linha é chamada de streampath). A ferramenta permite que os points sprites ou as linhas possam ser mapeados para cores por meio de uma função de transferência linear associada a um atributo. Já para a visualização do espaço de atributos os autores utilizaram a técnica Coordenadas Paralelas (CP), que também é utilizada para a interação com o usuário. Entretanto, a técnica CP é incapaz de mostrar a evolução temporal dos atributos. Dessa forma, eles utilizam uma estratégia baseada no gráfico 2D $x-y$, sendo o eixo vertical o atributo selecionado e o eixo horizontal o tempo. Embora o referido trabalho apresente uma estratégia para a investigação temporal dos atributos, ela é limitada a um único atributo por vez.

Wei, Yu, Grout, Chen e Ma (2012) apresentam o trabalho mais próximo da abordagem introduzida neste trabalho para visualizar as alterações nas relações de similaridade do espaço de atributos ao longo de toda a simulação em uma única visualização (Capítulo 5). A ferramenta desenvolvida por eles visualiza ambos os espaços simultaneamente (chamado de espaço dual), o espaço de atributos, chamado de espaço de fase, e o espaço do objeto, chamado de espaço físico. Para a visualização do espaço do objeto eles utilizam o rendering das polilinhas formadas pelos sucessivos posicionamento da partícula ao longo da simulação, correspondendo à trajetória da partícula.

A polilinha gerada no espaço de fase foi chamada de curva de evolução do atributo (attribute evolution curve). Entretanto, exibir todas as curvas dificultaria a percepção da evolução dos atributos. Desse modo, os autores desenvolveram uma estratégia baseada tanto no agrupamento 
das curvas, utilizando uma versão do $k$-means chamada de agrupamento baseado em modelos (model-based clustering), como na manipulação dos agrupamentos gerados, ou seja o usuários especialistas podem unir ou dividir os agrupamentos. A Figura 3.8 apresenta seis pares da visualização do espaço dual, referente à aplicação da técnica de agrupamento e à manipulação dos grupos pelos usuários.
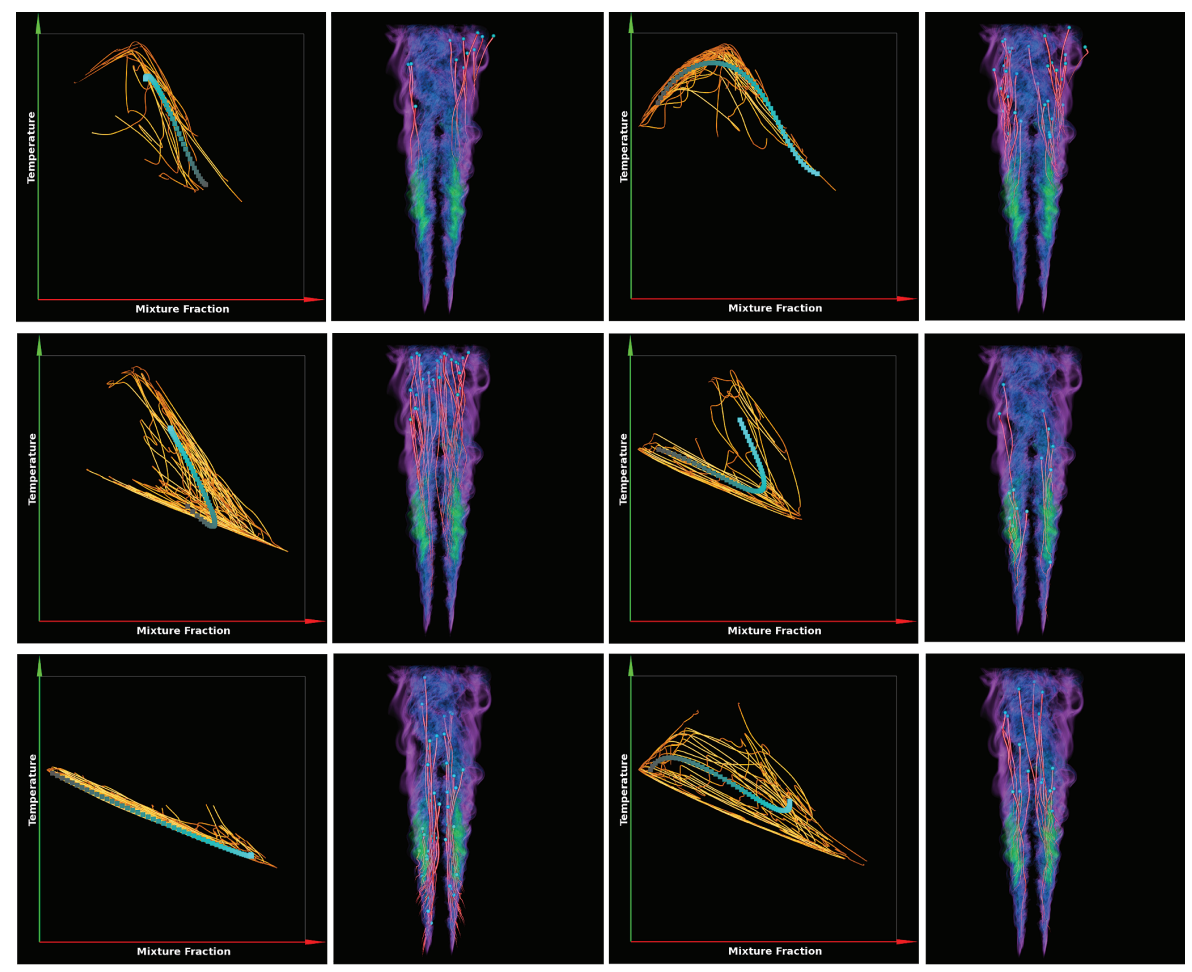

Figura 3.8: Visualização do espaço dual. Em cada par do espaço dual, a imagem a esquerda refere-se ao espaço de fase e a imagem a direta ao espaço físico. No espaço de fase, o eixo vertical corresponde a temperatura, já o eixo horizontal a fração de mistura de um conjunto de dados de simulação de combustível. Há seis pares do espaço dual, referente à aplicação da técnica de agrupamento e à manipulação pelo usuário. Fonte: (Wei et al., 2012)

A análise da curva de evolução do atributo é feita apenas sobre duas dimensões do conjunto de dados, já que a curva é gerada por meio do gráfico de dispersão 2D. Este fato constitui a maior limitação da técnica, ou seja, os demais atributos são ignorados ou outra análise deve ser feita. Ademais, a aplicação da técnica de agrupamento requer o número de grupos, a priori, e a manipulação dos agrupamentos requer um usuário com um certo conhecimento sobre o conjunto de dados.

\subsection{Considerações finais}

Este Capítulo apresentou os principais trabalhos que lidam com volumes multivalorados, muitos deles constituídos de sistemas de exploração visual. Foram considerados trabalhos que tratam os três tipos de volumes (voxels, células e partículas). Dos trabalhos analisados, nenhum 
foi capaz de fornecer uma visualização temporal de todo o espaço de atributos. Os trabalhos que mais se aproximaram foram os de Jones et al. (2008) e Wei et al. (2012), porém as estratégias definidas por eles estão limitadas a análise temporal de um ou dois atributos, respectivamente. 


\section{Visualização de malhas volumétricas variantes no tempo apoiada por projeções}

\subsection{Considerações iniciais}

Gerar visualizações de dados volumétricos multivalorados variantes no tempo utilizando projeções multidimensionais apresenta dois grandes desafios. Primeiro, projetar uma sequência de volumes garantindo interatividade e coordenação com uma representação do espaço do objeto requer a satisfação de requisitos como escalabilidade, precisão e coerência temporal e espacial. O segundo desafio é como aproveitar a visualização no espaço de atributos para transmitir informações relevantes sobre os volumes variantes no tempo. Neste Capítulo são investigadas abordagens para tratar estes problemas, considerando o objetivo de visualizar simultaneamente o espaço do objeto e o espaço de atributos associados a malhas volumétricas, definidas em termos de voxels ou células.

Foram consideradas dois cenários distintos: um conjunto de dados temporal formado por volumes regulares de voxels, composto por atributos escalares e um campo vetorial; e um conjunto de dados temporal que descreve um campo vetorial definido sobre um volume irregular. Esses conjuntos de dados temporais foram disponibilizados para a competição Viscontest, associada à IEEE Visualization Conference (ou IEEE VisWeek). O VisContest tem por objetivo promover a comparação de novas técnicas com as estabelecidas, fornecer parâmetros para a comunidade científica e criar um fórum de discussão na conferência.

No volume regular, as projeções do espaço de atributos consideraram apenas os atributos escalares: foram utilizados os campos escalares originais e campos escalares adicionais derivados 
do campo vetorial. Para a visualização no espaço do objeto foi utilizado o rendering volumétrico direto (Manssour, 2002). No volume irregular aplicou-se uma estratégia diferente, com base no campo vetorial: a visualização no espaço do objeto utiliza streamlines (Telea, 2008), a qual é associada uma visualização de um espaço de características extraídas das streamlines para apoiar a interação com a representação do espaço do objeto. Em ambos os casos, os espaços definidos pelos vetores de atributos foram projetados com a técnica PLMP, devido à sua capacidade de preservar a coerência espaço-temporal.

Este Capítulo está organizado de modo a descrever as funcionalidades implementadas para explorar esses conjuntos de dados, discutidos nas Seções 4.2 .2 e 4.3, respectivamente. A aplicação dessas funcionalidades em cenários representativos encontrados na visualização de dados gerados por simulações multidimensionais e de escoamentos de fluidos, que se caracterizam por serem definidos sobre domínios volumétricos e temporais, impondo diversas dificuldades para viabilizar cenários exploratórios, é apresentada nas seções 4.4 e 4.5.

\subsection{Investigação temporal de malhas volumétricas}

\subsubsection{Explorando agrupamentos}

Neste trabalho buscamos estratégias capazes de ajudar um usuário a identificar estruturas relevantes nos diferentes volumes, ao longo do tempo. Espera-se que a projeção dos elementos do volume seja útil na identificação de grupos de elementos com comportamento similar. Entretanto, devido à alta densidade de pontos na projeção, a percepção de grupos e estruturas, assim como a interação, são dificultadas.

Uma estratégia para favorecer a interação é aplicar técnicas de agrupamento capazes de identificar automaticamente os grupos de elementos similares, a cada volume da simulação. Desse modo, utiliza-se as técnicas descritas na Seção 2.6 para identificar agrupamentos. Como é difícil estabelecer, a priori, qual a técnica mais adequada, aplica-se diferentes algoritmos e escolhe-se, para apresentar ao usuário, o resultado que gera grupos com melhor coeficiente de silhueta (Seção 2.7).

Os agrupamentos podem ser detectados considerando os dados no espaço multidimensional original ou no espaço projetado. A aplicação sobre o layout da projeção objetiva favorecer a interação do usuário com a projeção e a percepção dos grupos formados na projeção - mas não necessariamente é fiel ao que acontece no espaço original. A aplicação diretamente sobre o espaço multidimensional pode ser interessante, mas tem um custo computacional maior.

A principal limitação e dificuldade é que as técnicas de agrupamento estão sendo aplicadas independentemente, em cada volume da sequência temporal. Assim, nem sempre é possível rastrear a evolução dos grupos ao longo do tempo, pois não há uma correspondência automática entre os grupos localizados em instantes de tempo distintos, e mesmo o número de grupos identificados pode variar ao longo do tempo. Na prática, em muitas situações é possível 
acompanhar visualmente a evolução dos agrupamentos. O ideal seria aplicar uma técnica de agrupamento temporal - mas um dos grandes desafios é o custo computacional, em se tratando de sequências de volumes.

\subsubsection{Explorando o comportamento temporal}

Partimos da suposição que a variação das relações de similaridade definidas no espaço de atributos, no decorrer do tempo, pode revelar informações importantes para o entendimento do fenômeno estudado. Portanto, ao identificar regiões com alta ou baixa variação dessas relações, em volumes subsequentes, espera-se auxiliar o usuário no entendimento do problema estudado. Dessa forma, foi implementada uma estratégia para identificar essas regiões, a qual pode ser sub-dividida em três etapas:

1. Inicia-se projetando o espaço de atributos do volume referente ao instante de tempo atual no espaço visual $\left(R^{2}\right)$. Cada ponto projetado descreve os atributos de uma primitiva (voxels ou células).

2. Compara-se, ponto a ponto, o deslocamento sofrido entre o momento anterior e o atual, usando-se a distância Euclidiana;

3. Os pontos (espaço de atributos) e as primitivas (espaço do objeto) são, então, classificados em função do deslocamento ocorrido, por meio da definição dos valores das faixas de variação de deslocamento.

Os valores das faixas de variação deslocamento correspondem a um conjunto com vários intervalos, os quais são utilizados para segmentar as representações no espaço de atributos e no espaço do objeto. O mecanismo funciona em três passos distintos. Primeiro, o usuário define quantos intervalos (faixas) deseja. Segundo, delimita-se às faixas por meio de um painel deslizante (lado direito na Figura 4.1). Cada faixa abrange uma região da projeção em um formato circular portanto, quando um ponto da projeção sofre uma variação cujo raio está dentro de uma determinada faixa, então o ponto será classificado para a faixa especificada. Terceiro, aplica-se esses processo para segmentar toda a projeção e, consequentemente, todo o espaço do objeto.

A Figura 4.1 apresenta um exemplo de configuração dos valores de deslocamento. As 5 circunferências delimitam os limiares de 6 faixas de valores, os quais podem ser definidas pelo usuário na interface a direita.

Os elementos classificados na primeira faixa (faixa A) são aqueles com os menores deslocamentos. No exemplo da Figura 4.1, os elementos da faixa A são todos os pontos que se deslocaram até 50 pixels. Já aqueles classificados na faixa B possuem deslocamento entre 50 e 100. Os pontos classificados na faixa $\mathrm{F}$ são aqueles cujo deslocamento está acima de 250 pixels.

Uma vez que todos os pontos foram classificados, então a projeção do volume é colorida de forma a refletir as faixas de deslocamento. As imagens da Figura 4.4 (coluna à esquerda) exibem 


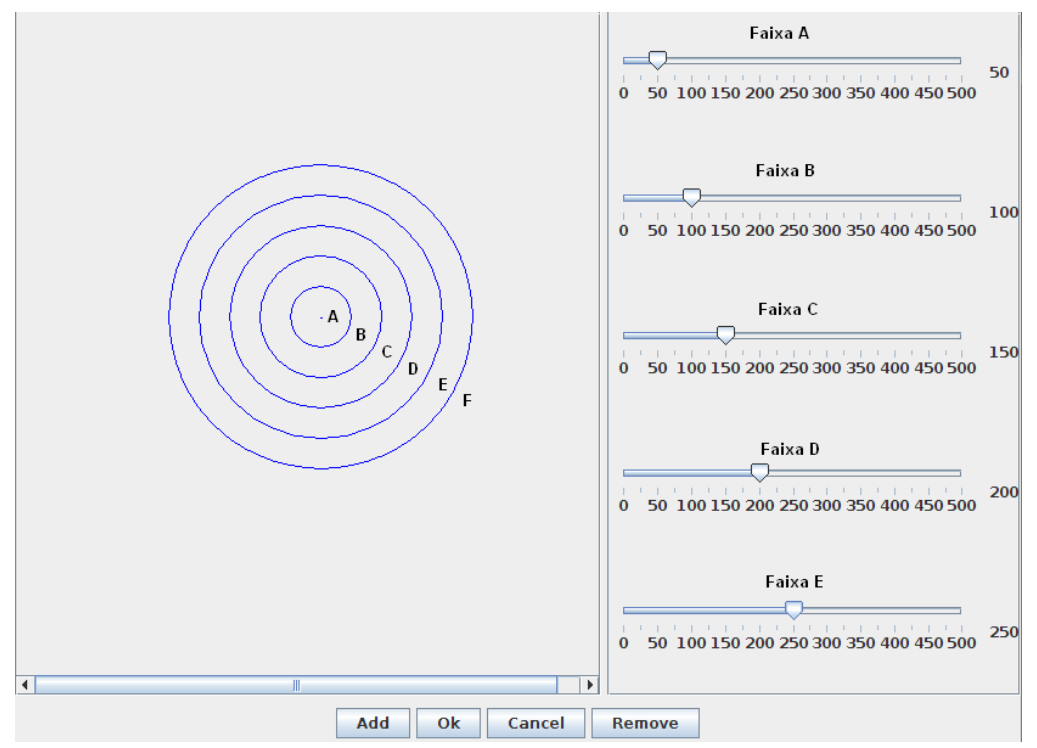

Figura 4.1: Exemplo de configuração dos intervalos de variação de deslocamento com 6 faixas. Os intervalos são delimitados pelos limiares indicados nas circunferências exibidas à esquerda. O raio da faixa pode ser modificado interagindo com barra de rolagem a direita.

projeções segmentadas em 4 faixas de valores. Uma faixa específica (faixa B) foi selecionada, e por isso aparece destacada na projeção (borda cinza ao redor dos pontos). Essa seleção é refletida no espaço do objeto por meio da coordenação entre ambas as visualizações, o que portanto permite localizar a região desses pontos no espaço físico.

Alternativamente, ao invés de calcular o deslocamento dos pontos no espaço projetado, o deslocamento pode ser calculado diretamente no espaço multidimensional dos dados. Isso requer uma normalização dos dados, como todos os atributos variando de 0 a 1 . Após a normalização, os intervalos de deslocamento podem ser definidos em relação ao deslocamento máximo observado. Considere dois elementos do conjunto de dados, os quais serão chamados de elementos extremos $\left(e_{1}, e_{2}\right)$, sendo $e_{1}=\{0,0, \ldots, 0\}_{1 \times d}$ e $e_{2}=\{1,1, \ldots, 1\}_{1 \times d}$. Dessa forma, pode-se afirmar que o deslocamento máximo, medido pela distância Euclidiana, seria:

$$
d_{\max }=\sqrt{1+1+\ldots+1}=\sqrt{d}
$$

Nesse caso, a comparação dos elementos é feita diretamente espaço original, ao invés de no espaço projetado. Ainda assim, a visualização da projeção continua sendo útil em outros aspectos, como a visualização da distribuição dos elementos nas faixas, a percepção da relação de proximidade entre os elementos de cada faixa, a localização da vizinhança e interação com o conjunto de dados no processo exploratório.

Por exemplo, a relação de vizinhança entre os elementos classificados nas faixas definidas pelo usuário. Pode exibir os agrupamentos existentes no conjunto de dados e verificar em quais faixas os elementos do agrupamento foram classificados. A projeção fornece uma percepção global das relações de similaridades entre os elementos e, dessa forma, pode exibir tendências com relação 
a determinadas faixas. Ou seja, pode ser que a faixa de menor deslocamento apareça sempre em uma mesma região da projeção. Ademias, a projeção multidimensional pode fornecer um meio simples e até mesmo intuitivo para interação do usuário ao explorar o conjunto de dados.

Uma dificuldade óbvia é encontrar o melhor valor para os limiares das faixas que segmentam a projeção. Como esses limiares estão relacionados ao deslocamento sofrido entre dois volumes consecutidos, então os valores adequados precisam ser determinadas para cada conjunto de dados ou simulação.

\subsection{Investigação temporal de campos vetoriais}

Uma técnica bastante utilizada para a visualização de dados vetoriais volumétricos são as streamlines (Telea, 2008). Uma streamline descreve o caminho percorrido por uma partícula abstrata em um campo vetorial, partindo de um determinado local chamado de semente (seed), a qual é conduzida pela "correnteza" advinda dos valores vetoriais. Em termos matemáticos, uma streamline pode ser descrita como uma integração do campo vetorial $\mathbf{v}$ sobre algum intervalo temporal $T$. A partícula abstrata é integrada a partir de uma posição $p_{0}$ definida pelo usuário.

$$
S=\{p(\tau), \tau \in[0, T]\}, p(\tau)=\int_{t=0}^{\tau} \mathbf{v}(p) d t
$$

sendo $p(0)=p_{0}$. É importante ressaltar que as streamlines são geradas para atributos vetoriais independentes do tempo. De fato, na equação 4.2 o tempo $t$ representa o tempo de integração e não o tempo físico do conjunto de dados (Telea, 2008).

A visualização das streamlines favorece a percepção do deslocamento provocado pelo campo vetorial estudado em um instante de tempo específico. A Figura 4.6 apresenta um exemplo de visualização de streamlines. Cada polilinha representa uma streamline. Todas as streamlines foram traçadas com o mesmo intervalo de integração $T$, mas algumas possuem diferentes comprimentos, já que o campo vetorial apresenta grandes variações. Durante o traçado da streamline o algoritmo encerra em duas condições: quando alcança a fronteira do conjunto de dados ou quando excede o tempo máximo.

Segundo Telea (2008), várias considerações técnicas surgem quando se utiliza as streamlines. A primeira preocupação diz respeito à discretização da equação 4.2, com a aplicação dos métodos numéricos de integração. Com frequência, tal equação pode ser computada utilizando-se a integração de Euler ou o método de Runge-Kutta (Burden e Douglas Faires, 2001), sendo que o primeiro é mais rápido, porém menos preciso. Entretanto, métodos numéricos apresentam erros cumulativos à medida que o tempo $\tau$ aumenta. Já que as posições ao longo da streamline são computadas incrementalmente, isso significa que as extremidades tendem a desviar da posição correta. Neste trabalho utilizamos como base a implementação disponível na biblioteca VTK (The Visualization Toolkit) ${ }^{1}$ e o método de Runge-Kutta, por ser mais preciso.

\footnotetext{
${ }^{1}$ http://www.vtk.org/
} 
Por fim, um dos fatores cruciais para a visualização das streamlines é a escolha da posição inicial (semente) e o número delas. Uma maneira de utilizar streamlines é povoar uma região de interesse com várias sementes e gerar o traçado. Porém, mesmo que a região esteja densamente povoada com sementes, isso não garante que todo o conjunto de dados será coberto pelas polilinhas. Uma segunda maneira consiste em distribuir de maneira uniforme várias sementes pelo conjunto de dados e então gerar as streamlines em ambas as direções - nste trabalho aplicamos esse procedimento em todas as visualizações de streamlines.

\subsubsection{Extração do vetor de características}

Para projetar uma streamline (ou uma streamfeature ou streampath, como discutido no Capítulo 5) é necessário extrair um vetor de características multidimensionais que a descreva. O propósito de projetar as linhas é evidenciar aquelas que apresentam propriedades similares de forma ou geometria, de modo que a projeção apoie o usuário na tentativa de identificar grupos de comportamento similar, tendências e facilitar o processo interativo de exploração dessas entidades. Pode-se considerar, além do formato, propriedades geométricas como o posicionamento inicial, o final e o tamanho da linha.

De maneira similar ao trabalho de Poco et al. (2012), gera-se um vetor de características a partir dos coeficientes de alta e baixa frequência da transformada de Fourier das linhas. No nosso caso, usamos uma FFT (Reddy e Chatterji, 1996) em cada direção espacial (x,y,z). Adicionalmente, pode-se utilizar informações geométricas, como a posição inicial e final da linha, ou o seu tamanho e centro de massa. Consideramos, neste trabalho, vetores de características com até 40 atributos: 30 coeficientes de Fourier (sendo 15 de baixa e 15 de alta frequência, composto de 5 coeficiente para cada eixo $[\mathrm{x}, \mathrm{y}, \mathrm{z}]$ ) e 10 atributos geométricos (sendo 9 referentes as coordenadas em $[\mathrm{x}, \mathrm{y}, \mathrm{z}]$ do ponto inicial, ponto final e centro de massa - sendo 1 referente ao tamanho da linha).

Esse processo de extração de características gera, tipicamente, um vetor multidimensional dominado pelos atributos de forma (os coeficientes de Fourier). A utilização ou não das características geométricas depende do propósito da investigação: pode ser desejável se o objetivo é identificar polilinhas que, além de possuírem um formato semelhante, estão em uma mesma região espacial. Assim pode-se, dependendo dos objetivos, ignorar os atributos geométricos ou, ao contrário, aumentar a sua influência na representação, aumentando o seu peso relativo.

\subsection{Visualização exploratória: furacão Isabel}

As estratégias descritas acima foram aplicadas ao conjunto de dados disponibilizado para o Viscontest ${ }^{2}$ 2004, que corresponde a uma sequência de volumes de voxels que descrevem uma tormenta tropical que assolou o leste dos Estados Unidos em setembro de 2003, batizada de furacão Isabel. Quando se trata de visualizar dados de um furacão, um dos objetivos é identificar

\footnotetext{
${ }^{2}$ www.viscontest.org
} 
e rastrear a evolução do centro da tempestade, chamado de olho do furacão, o qual pode ser percebido nos cenários a seguir.

Os volumes incluem os atributos listados na Tabela 4.1. Além dos atributos escalares advindos da simulação, foram incorporados 6 atributos extraídos do campo vetorial (velocidade). Os volumes foram reduzidos - cada volume de dimensões 500x500x100 foi reduzido para $125 \times 125 \times 25$, computando o valor médio a cada sub-volume de dimensões $4 \mathrm{x} 4 \mathrm{x} 4$, nos 48 instantes de tempo de modo a torná-los manipuláveis.

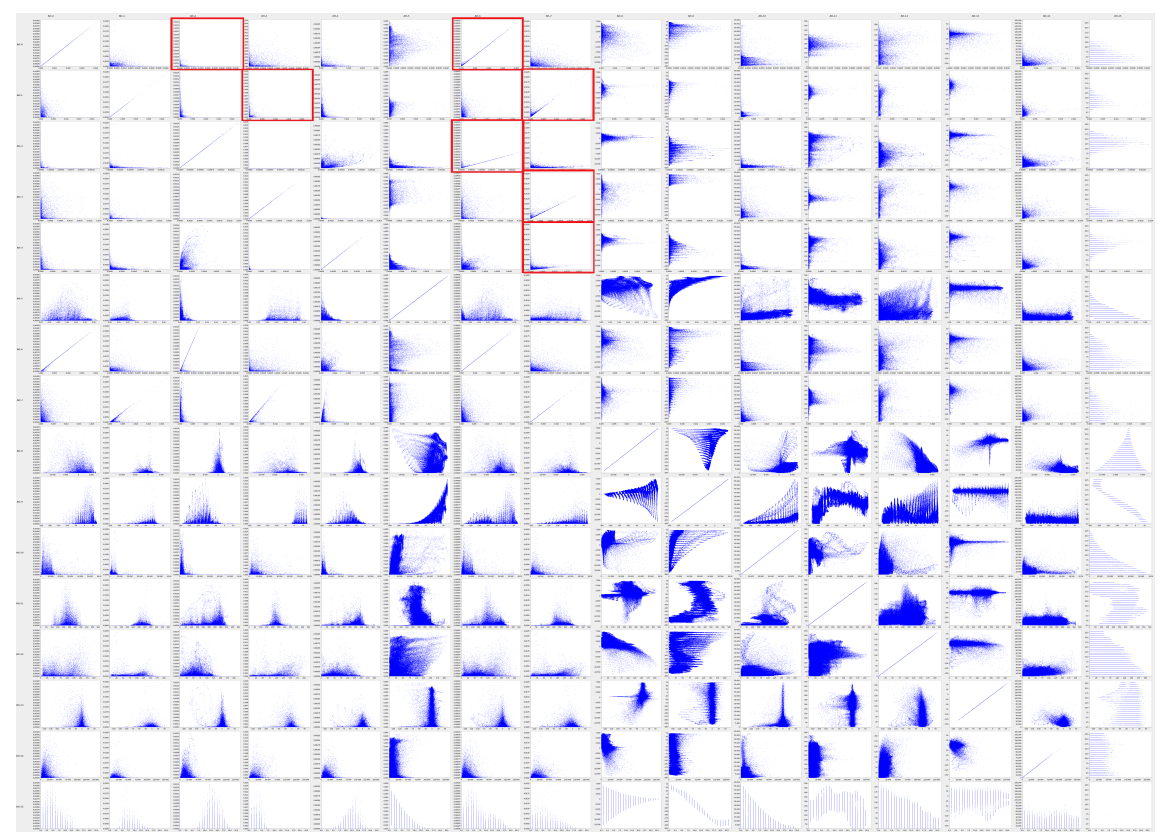

Figura 4.2: Matriz de gráficos de dispersão dos 16 atributos do conjunto de dados do furacão. Em destaque os atributos que aparentam possuir uma correlação linear.

A Figura 4.2 exibe a matriz de gráficos de dispersão, destacando-se os atributos que "aparentemente" apresentam algum tipo de correlação linear(uma distribuição em linha reta), as quais estão identificadas na Tabela 4.1. Atributos linearmente correlacionados foram eliminados (mantendo-se apenas um), o que ocasionou uma redução para 12 atributos, cuja numeração final é apresentada na referida tabela.

A sequência de volumes foi investigada aplicando as duas estratégias já discutidas: explorando agrupamentos e investigando as variações temporais no espaço de atributos.

\section{Explorando agrupamentos}

Relembrando que o propósito da investigação via agrupamentos é ajudar na percepção dos grupos de elementos similares em um instante de tempo específico, e tentar acompanhar a evolução dos agrupamentos ao longo da simulação. Foram aplicadas as três técnicas de agrupamento capazes de localizar o melhor número de agrupamentos (X-means, bisecting X-means e $x$-medoid). Entretanto, não foi possível a utilização do $x$-medoid devido ao custo quadrático 
38 Capítulo 4. Visualização de malhas volumétricas variantes no tempo apoiada por projeções

\begin{tabular}{|c|c|c|c|}
\hline Numeração & Atributo & Correlação com & Numeração Final \\
\hline Att-0 & porção de água nas nuvens & Att-2, Att-6 & Att-0 \\
Att-1 & precipitação de gelo & Att-3, Att-7 & Att-1 \\
Att-2 & porção de gelo nas nuvens & Att-0, Att-6 & - \\
Att-3 & chuva & Att-1, Att-7 & Att-2 \\
Att-4 & porção de neve & Att-7 & Att-3 \\
Att-5 & porção do vapor d'água & - & - \\
Att-6 & total da porção de gelo e neve & Att-0, Att-2 & Att-4 \\
Att-7 & total da precipitação de chuva, neve e gelo & Att-1, Att-3, Att-4 & Att-5 \\
Att-8 & pressão atmosférica & - & Att-6 \\
Att-9 & temperatura em Celsius & - & Att-7 \\
Att-10 & gradiente da pressão & - & Att-8 \\
Att-11 & gradiente da temperatura & - & Att-9 \\
Att-12 & magnitude do vento & - & Att-10 \\
Att-13 & divergência do vento & - & Att-11 \\
Att-14 & ondulação do vento & - & \\
Att-15 & altura do voxel & & \\
\hline
\end{tabular}

Tabela 4.1: Atributos do furacão com a identificação dos atributos correlacionados, e dos selecionados, com a numeração final.

da técnica, haja vista o elevado tamanho de cada volume (390.625 voxels). Foram utilizados os mesmos parâmetros para as duas técnicas (número de agrupamento procurados entre $10 \mathrm{e}$ 50). O resultado é mostrado na Tabela 4.2. O X-means gera os melhores valores de silhueta, portanto foi a técnica selecionada para a investigação temporal.

\begin{tabular}{|c|c|c|c|}
\hline Tempo & Técnica & Valor da Silhueta & $\mathrm{N}^{\circ}$ de Agrupamento \\
\hline 01 & X-means & $\mathbf{0 . 2 4 8 1}$ & 20 \\
01 & bisecting X-means & 0.0811 & 50 \\
\hline 25 & X-means & $\mathbf{0 . 1 9 1 6}$ & 20 \\
25 & bisecting X-means & 0.0044 & 50 \\
\hline
\end{tabular}

Tabela 4.2: Aplicação das técnicas de agrupamento no início e no meio da simulação (01 e 25). O X-means obteve os melhores resultados.

Durante a exploração do conjunto de dados é possível verificar a evolução dos agrupamentos localizados pelo $X$-means, ao longo da simulação. Apresenta-se nesta seção apenas um dos agrupamentos, em 4 instantes de tempos distintos.

O X-means localizou, no instante cinco, 20 agrupamentos, porém no instante 15, a técnica (com os mesmos parâmetros) identificou 19 grupos. Nos demais instantes de tempo (25 e 35) a técnica localizou 20 agrupamentos.

É possível observar nas imagens das projeções da Figura 4.3 (coluna à esquerda) que a primeira projeção (tempo cinco) possui uma coloração diferente das demais: nesse momento, o $X$-means enumerou o grupo com o valor diferente dos outros instantes de tempo.

Na Tabela 4.3 tem-se a evolução dos pontos no agrupamento em análise. Percebe-se que no instante 15 há uma maior concentração de pontos nesse grupo do que nos demais momentos da simulação. 
Observando as imagens do espaço do objeto da Figura 4.3 (coluna da direita), percebe-se que o agrupamento selecionado apresenta uma forma parecida com o funil típico do olho do furacão.

\begin{tabular}{|c|c|c|}
\hline Tempo & $\mathrm{N}^{\circ}$ de Pontos & Porcentagem em Relação ao Volume \\
\hline 05 & 10.280 & $2,63 \%$ \\
15 & 17.538 & $4,49 \%$ \\
25 & 14.383 & $3,68 \%$ \\
35 & 13.230 & $3,39 \%$ \\
\hline
\end{tabular}

Tabela 4.3: Evolução dos pontos pertencentes ao agrupamento destacado na Figura 4.3.

É possível observar a evolução dos atributos dos elementos do grupo, ao longo da simulação, por meio das Coordenadas Paralelas. Nas imagens da coluna do meio da Figura 4.3, pode-se verificar essa modificação. É de fácil percepção, pelas imagens, que os seguintes atributos sofrem maior modificação: temperatura (Att-5), magnitude do vento (Att-8), divergência da velocidade do vento (Att-9) e altura (Att-11).

\section{Investigação temporal}

A estratégia de investigação temporal foi aplicada, nesse caso sobre o espaço de dados multidimensionais. Em outros experimentos realizados neste trabalho, os resultados obtidos no espaço projetado foram semelhantes aos obtidos diretamente sobre o espaço multidimensional.

Foram atribuídos os valores para os limites que definem os limiares de cada faixa segundo a Tabela 4.4, calculados proporcionalmente em relação ao deslocamento máximo, que nesse caso é $\sqrt{12}$.

\begin{tabular}{|c|c|c|c|}
\hline Faixa A & Faixa B & Faixa C & Faixa D \\
\hline $0 \%$ a $4 \%$ & $4 \%$ a $8 \%$ & $8 \%$ a $12 \%$ & $12 \%$ a $100 \%$ \\
\hline
\end{tabular}

Tabela 4.4: Atribuição dos limites para as quatro faixas definidas para segmentar o volume.

Os pontos classificados na faixa A são aqueles que quase não variam e serão chamados de pontos de fundo, já os pontos da faixa D são aqueles com as maiores mudanças e serão chamados de pontos do topo. Por motivos de espaço, será exibido aqui apenas os pontos na faixa B.

Na Tabela 4.5 mostra a quantidade de pontos por faixa e sua porcentagem em relação ao conjunto nos 4 instantes de tempo $(05,15,25$ e 35). Observa-se que a faixa A (pontos de fundo) tem a grande maioria dos pontos, sempre acima de $90 \%$. Já os pontos da faixa B, os quais são detalhados nas imagens da Figura 4.4, apresentam maior concentração no instante 05.

Ao analisar as imagens das projeções na Figura 4.4 (coluna à esquerda), percebe-se que os pontos destacados (faixa B) estão distribuídos por toda a área da projeção. Nas imagens da coluna do meio da Figura 4.4 é possível perceber que os atributos pouco variam durante os 4 instantes de tempo exibidos. Deve-se levar em consideração a diminuição de quase $50 \%$ dos pontos constituintes dessa faixa, já que ela inicia com 15.106 pontos e finaliza com 8.868 pontos, nos instantes 05 e 35 respectivamente. 
40 Capítulo 4. Visualização de malhas volumétricas variantes no tempo apoiada por projeções

\begin{tabular}{|c|c|c|c|c|}
\hline Tempo & Faixa A & Faixa B & Faixa C & Faixa D \\
\hline 05 & $95,09 \%(371.438)$ & $3,87 \%(15.106)$ & $0,78 \%(3.031)$ & $0,27 \%(1.050)$ \\
15 & $96,90 \%(378.526)$ & $2,74 \%(10.688)$ & $0,29 \%(1.137)$ & $0,07 \%(274)$ \\
25 & $97,07 \%(379.187)$ & $2,50 \%(9.756)$ & $0,34 \%(1.338)$ & $0,09 \%(344)$ \\
35 & $97,27 \%(379.979)$ & $2,27 \%(8.868)$ & $0,35 \%(1.349)$ & $0,11 \%(429)$ \\
\hline
\end{tabular}

Tabela 4.5: Distribuição dos dados em relação as faixas de variação ao longo da simulação do furacão.

Ao analisar as imagens do espaço do objeto, coluna à direita da Figura 4.4, percebe-se que a faixa B está concentrada em três locais: o olho do furacão, uma região cujo formato aparenta ser uma semi-circunferência e em alguns pontos distribuídos pelo volume.

\subsection{Visualização exploratória: bomba hidráulica}

O Viscontest 2011 abordou o problema da visualização de volumes irregulares, nesse caso advindas de simulações de dinâmica dos fluidos sobre uma bomba hidráulica. Na competição o interesse era em visualizações capazes de ajudar a entender o movimento e o desenvolvimento dos vórtices no escoamento. Por esse motivo, elegeu-se trabalhar com o campo vetorial utilizando a visualização de streamlines, já que uma streamline "vortical" é caracterizada pelo seu formato peculiar, assemelhando-se a uma curva helicoidal.

Definiu-se como objetivo a localização e o agrupamento das streamlines "vorticais", procurando identificar e rastrear a evolução de tais entidades de interesse. A seguir tem-se a descrição do conjunto de dados, e em seguida, os resultados obtidoscom as duas estratégias de investigação (via agrupamentos e temporal). Os resultados deste estudo foram submetidos ao IEEE Viscontest 2011 (Santos et al., 2011).

O conjunto de dados da bomba hidráulica é constituído por 80 volumes irregulares com aproximadamente 2 milhões de vértices por volume. Vale ressaltar nenhuma redução foi aplicada para os volumes desse conjunto. A estratégia utilizada para visualizar os vórtices é baseada na visualização do atributo vetorial (velocidade), por meio da visualização das streamlines, juntamente com as projeções das características extraídas dessas polilinhas.

Os atributos extraídos das streamlines estão enumerados na Tabela 4.6, juntamente com a identificação dos atributos altamente correlacionados e a seleção e numeração final para a investigação. A identificação dos atributos altamente correlacionados foi feita com base na matriz de gráficos de dispersão na Figura 4.5.

\section{Explorando agrupamentos}

Foram aplicadas no início e no meio da simulação as duas técnicas de agrupamento $(X$-means e bisecting $X$-means) sobre os dados projetados, os resultados estão na Tabela 4.7. Vale ressaltar que ao invés de investigar os agrupamentos diretamente no espaço multidimensional, como na investigação do furacão Isabel, dessa vez, a investigação foi aplicada no espaço projetado. 


\begin{tabular}{|c|c|c|c|}
\hline Numeração & Atributo & Correlação com & Numeração Final \\
\hline Att-0 & coeficiente $\mathrm{n}^{\circ} 0$ de Fourier mínimo em $\mathrm{x}$ & Att-36 & Att-0 \\
\hline Att-1 & coeficiente $\mathrm{n}^{\circ} 1$ de Fourier mínimo em $\mathrm{x}$ & Att-9 & Att-1 \\
\hline Att-2 & coeficiente $\mathrm{n}^{\circ} 2$ de Fourier mínimo em $\mathrm{x}$ & Att-8 & Att-2 \\
\hline Att-3 & coeficiente $\mathrm{n}^{\circ} 3$ de Fourier mínimo em $\mathrm{x}$ & Att-7 & Att-3 \\
\hline Att-4 & coeficiente $\mathrm{n}^{\circ} 4$ de Fourier mínimo em $\mathrm{x}$ & Att-6 & Att-4 \\
\hline Att-5 & coeficiente $\mathrm{n}^{\circ} 0$ de Fourier máximo em $\mathrm{x}$ & - & Att-5 \\
\hline Att-6 & coeficiente $\mathrm{n}^{\circ} 1$ de Fourier máximo em $\mathrm{x}$ & Att-4 & - \\
\hline Att-7 & coeficiente $\mathrm{n}^{\circ} 2$ de Fourier máximo em $\mathrm{x}$ & Att-3 & - \\
\hline Att-8 & coeficiente $\mathrm{n}^{\circ} 3$ de Fourier máximo em $\mathrm{x}$ & Att-2 & - \\
\hline Att-9 & coeficiente $\mathrm{n}^{\circ} 4$ de Fourier máximo em $\mathrm{x}$ & Att-1 & - \\
\hline Att-10 & coeficiente $\mathrm{n}^{\circ} 0$ de Fourier mínimo em y & Att-37 & Att-6 \\
\hline Att-11 & coeficiente $\mathrm{n}^{\circ} 1$ de Fourier mínimo em y & Att-19 & Att-7 \\
\hline Att-12 & coeficiente $\mathrm{n}^{\circ} 2$ de Fourier mínimo em y & Att-18 & Att-8 \\
\hline Att-13 & coeficiente $\mathrm{n}^{\circ} 3$ de Fourier mínimo em y & Att-17 & Att-9 \\
\hline Att-14 & coeficiente $\mathrm{n}^{\circ} 4$ de Fourier mínimo em y & Att-16 & Att-10 \\
\hline Att-15 & coeficiente $n^{\circ} 0$ de Fourier máximo em y & - & Att-11 \\
\hline Att-16 & coeficiente $n^{\circ} 1$ de Fourier máximo em y & Att-14 & - \\
\hline Att-17 & coeficiente $\mathrm{n}^{\circ} 2$ de Fourier máximo em y & Att-13 & - \\
\hline Att-18 & coeficiente $n^{\circ} 3$ de Fourier máximo em y & Att-12 & - \\
\hline Att-19 & coeficiente $\mathrm{n}^{\circ} 4$ de Fourier máximo em y & Att-11 & - \\
\hline Att-20 & coeficiente $\mathrm{n}^{\circ} 0$ de Fourier mínimo em $\mathrm{z}$ & Att-38 & Att-12 \\
\hline Att-21 & coeficiente $\mathrm{n}^{\circ} 1$ de Fourier mínimo em $\mathrm{z}$ & Att-29 & Att-13 \\
\hline Att-22 & coeficiente $\mathrm{n}^{\circ} 2$ de Fourier mínimo em $\mathrm{z}$ & Att-28 & Att-14 \\
\hline Att-23 & coeficiente $\mathrm{n}^{\circ} 3$ de Fourier mínimo em $\mathrm{z}$ & Att-27 & Att-15 \\
\hline Att-24 & coeficiente $\mathrm{n}^{\circ} 4$ de Fourier mínimo em $\mathrm{z}$ & Att-26 & Att-16 \\
\hline Att-25 & coeficiente $\mathrm{n}^{\circ} 0$ de Fourier máximo em $\mathrm{z}$ & - & Att-17 \\
\hline Att-26 & coeficiente $\mathrm{n}^{\circ} 1$ de Fourier máximo em $\mathrm{z}$ & Att-24 & - \\
\hline Att-27 & coeficiente $\mathrm{n}^{\circ} 2$ de Fourier máximo em $\mathrm{z}$ & Att-23 & - \\
\hline Att-28 & coeficiente $\mathrm{n}^{\circ} 3$ de Fourier máximo em $\mathrm{z}$ & Att-22 & - \\
\hline Att-29 & coeficiente $\mathrm{n}^{\circ} 4$ de Fourier máximo em $\mathrm{z}$ & Att-21 & - \\
\hline Att-30 & ponto inicial em $\mathrm{x}$ & - & Att-18 \\
\hline Att-31 & ponto inicial em y & - & Att-19 \\
\hline Att-32 & ponto inicial em $\mathrm{z}$ & - & Att-20 \\
\hline Att-33 & ponto final em $\mathrm{x}$ & - & Att-21 \\
\hline Att-34 & ponto final em y & - & Att-22 \\
\hline Att-35 & ponto final em $\mathrm{z}$ & - & Att-23 \\
\hline Att-36 & centro de massa em $\mathrm{x}$ & Att-0 & - \\
\hline Att-37 & centro de massa em y & Att-10 & - \\
\hline Att-38 & centro de massa em z & Att-20 & — \\
\hline Att-39 & tamanho da streamline & - & Att-24 \\
\hline
\end{tabular}

Tabela 4.6: Listagem dos atributos da bomba hidráulica, com a identificação dos atributos correlacionados e a seleção para a numeração final.

As técnicas de agrupamento foram aplicadas com os mesmos parâmetros, buscando um número de grupos no intervalo [25,100]. O X-means apresentou os melhores valores de silhueta, portanto, foi selecionado para a investigação temporal.

No decorrer da investigação, o X-means foi aplicado em quatro instantes de tempo distintos (10, 30, 50 e 70). Um agrupamento específico é selecionado e acompanhado, cuja evolução pode ser observada nas imagens da Figura 4.6, como também na Tabela 4.8. 
Capítulo 4. Visualização de malhas volumétricas variantes no tempo apoiada por projeções

\begin{tabular}{|c|c|c|c|}
\hline Tempo & Técnica & Valor da Silhueta & $\mathrm{N}^{\circ}$ de Agrupamento \\
\hline 10 & $X$-means & $\mathbf{0 . 3 5 1 1 0 0}$ & 25 \\
10 & bisecting X-means & 0.302317 & 25 \\
\hline 30 & $X$-means & $\mathbf{0 . 3 5 2 4 5 3 0 8}$ & 25 \\
30 & bisecting X-means & 0.286605 & 25 \\
\hline
\end{tabular}

Tabela 4.7: Aplicação das técnicas de agrupamento no início e no meio da simulação (10 e $30)$ sobre os dados projetados, usando o intervalo [25,100]. O X-means obteve os melhores resultados.

\begin{tabular}{|c|c|c|}
\hline Tempo & $\mathrm{N}^{\circ}$ de Pontos & Porcentagem em Relação ao Volume \\
\hline 10 & 1.660 & $3,01 \%$ \\
30 & 1.596 & $3,76 \%$ \\
50 & 1.590 & $3,75 \%$ \\
70 & 1.497 & $3,53 \%$ \\
\hline
\end{tabular}

Tabela 4.8: Evolução dos pontos pertencentes ao agrupamento destacado na Figura 4.6 .

Para esse conjunto de dados, a técnica de agrupamento aplicada sempre localizou o mesmo número de grupos. Entretanto, persiste a dificuldade de estabelecer a correspondência entre os grupos: as imagens das projeções (coluna à esquerda) na Figura 4.6 apresentam o mesmo grupo destacado com cores diferentes, em instantes distintos.

Ao analisar as imagens das Coordenadas Paralelas da Figura 4.6 (coluna central), percebe-se que o traçado das linhas possui uma alta similaridade. A terceira imagem (instante de tempo 50) é ligeiramente diferente das outras três. Tal fato é refletido no espaço do objeto.

\section{Investigação temporal}

Mais uma vez, aplicou-se a investigação temporal a partir dos dados multidimensionais, já que dessa forma é possível medir o percentual das primitivas que variam de um instante para o outro. Foi atribuído os valores para os limites que definem os pontos de cada faixa conforme a Tabela 4.9. Vale relembrar que a porcentagem foi calculada em relação ao deslocamento máximo, que nesse caso é $\sqrt{25}$.

\begin{tabular}{|c|c|c|c|c|c|}
\hline Faixa A & Faixa B & Faixa C & Faixa D & Faixa E & Faixa $\mathrm{F}$ \\
\hline $0 \%$ a $5 \%$ & $5 \%$ a $10 \%$ & $10 \%$ a $15 \%$ & $15 \%$ a $20 \%$ & $20 \%$ a $25 \%$ & $25 \%$ a $100 \%$ \\
\hline
\end{tabular}

Tabela 4.9: Atribuição dos limites para as quatro faixas definidas para segmentar o volume.

Na Tabela 4.10 tem-se a quantidade de pontos por faixa e sua porcentagem em relação ao conjunto nos 4 instantes de tempo (11,31, 51 e 71). A investigação foi desenvolvida sobre os pontos da faixa $\mathrm{F}$, os pontos de maior variação, apresentados nas imagens da Figura 4.7.

Ao analisar as imagens das projeções na Figura 4.7 (coluna à esquerda), percebe-se que os pontos destacados (faixa $\mathrm{F}$ - pontos na cor vermelha) estão distribuídos por toda a área da projeção. Já nas imagens das Coordenadas Paralelas (coluna do meio da Figura 4.7), é possível perceber que há uma predominância de um padrão, embora diferente em cada instante 


\begin{tabular}{|c|c|c|c|c|c|c|}
\hline Tempo & Faixa A & Faixa B & Faixa C & Faixa D & Faixa E & Faixa F \\
\hline 11 & $33,03 \%(14.007)$ & $26,85 \%(11.386)$ & $22,13 \%(9.386)$ & $12,54 \%(5.320)$ & $4,21 \%(1.785)$ & $1,24 \%(524)$ \\
31 & $30,77 \%(13.053)$ & $27,04 \%(11.472)$ & $22,54 \%(9.563)$ & $13,66 \%(5.796)$ & $4,57 \%(1.940)$ & $1,42 \%(604)$ \\
51 & $32,44 \%(13.754)$ & $26,32 \%(11.159)$ & $22,05 \%(9.349)$ & $13,58 \%(5.759)$ & $4,24 \%(1.799)$ & $1,37 \%(579)$ \\
71 & $21,48 \%(9.111)$ & $30,65 \%(13.001)$ & $25,46 \%(10.798)$ & $14,96 \%(6.343)$ & $5,51 \%(2.336)$ & $1,94 \%(823)$ \\
\hline
\end{tabular}

Tabela 4.10: Distribuição dos dados em relação as faixas de variação ao longo da simulação da Bomba Hidráulica.

de tempo. A faixa analisada permanece com certa estabilidade de elementos, em torno de 1,40\% do conjunto, sendo que no último instante investigado (71) há uma elevação considerável $(1,94 \%)$.

Ao analisar as imagens do espaço do objeto, coluna à direita da Figura 4.7, percebe-se que a distribuição das streamlines com a maior mudança de similaridade permeia todo o volume, não havendo concentração em nenhuma região específica.

\subsection{Considerações finais}

Este capítulo apresentou as duas abordagens desenvolvidas neste trabalho para visualizar simultaneamente o espaço do objeto e o espaço de atributos em volumes de malhas (voxels e células). Uma abordagem objetiva evidenciar a percepção para os grupos formados na projeção ou evidenciar os grupos advindos do conjunto de dados. A segunda abordagem procura evidenciar as alterações temporais entre dois instantes consecutivos, por meio da segmentação do volume entre os elementos que mais variaram daqueles de menor variação. Apresentou-se, também, a visualização das streamlines e o método de extração de característica de tais polilinhas. Por fim, mostrou-se os resultados obtidos utilizando-se as duas abordagens em conjunto de dados de volumes regulares e volumes irregulares. 

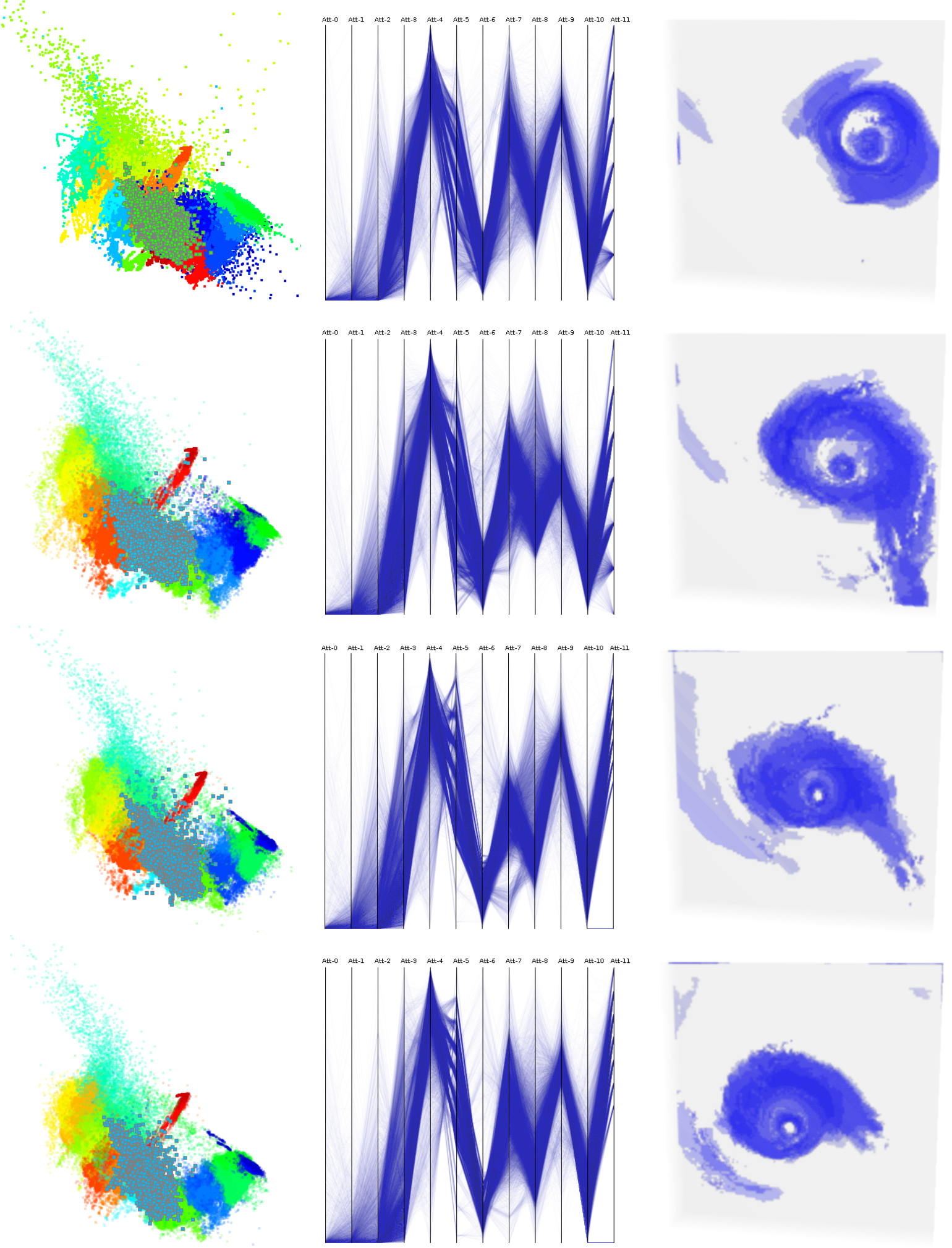

Figura 4.3: Na coluna à esquerda tem-se as projeções em quatro instantes de tempo $(05,15$, 25 e 35). Os pontos destacados nas projeções correspondem a um dos agrupamentos localizados pelo $X$-means. Na coluna do meio tem-se as Coordenadas Paralelas para o agrupamento destacado, e na coluna à direita tem-se a sua representação no espaço do objeto. 

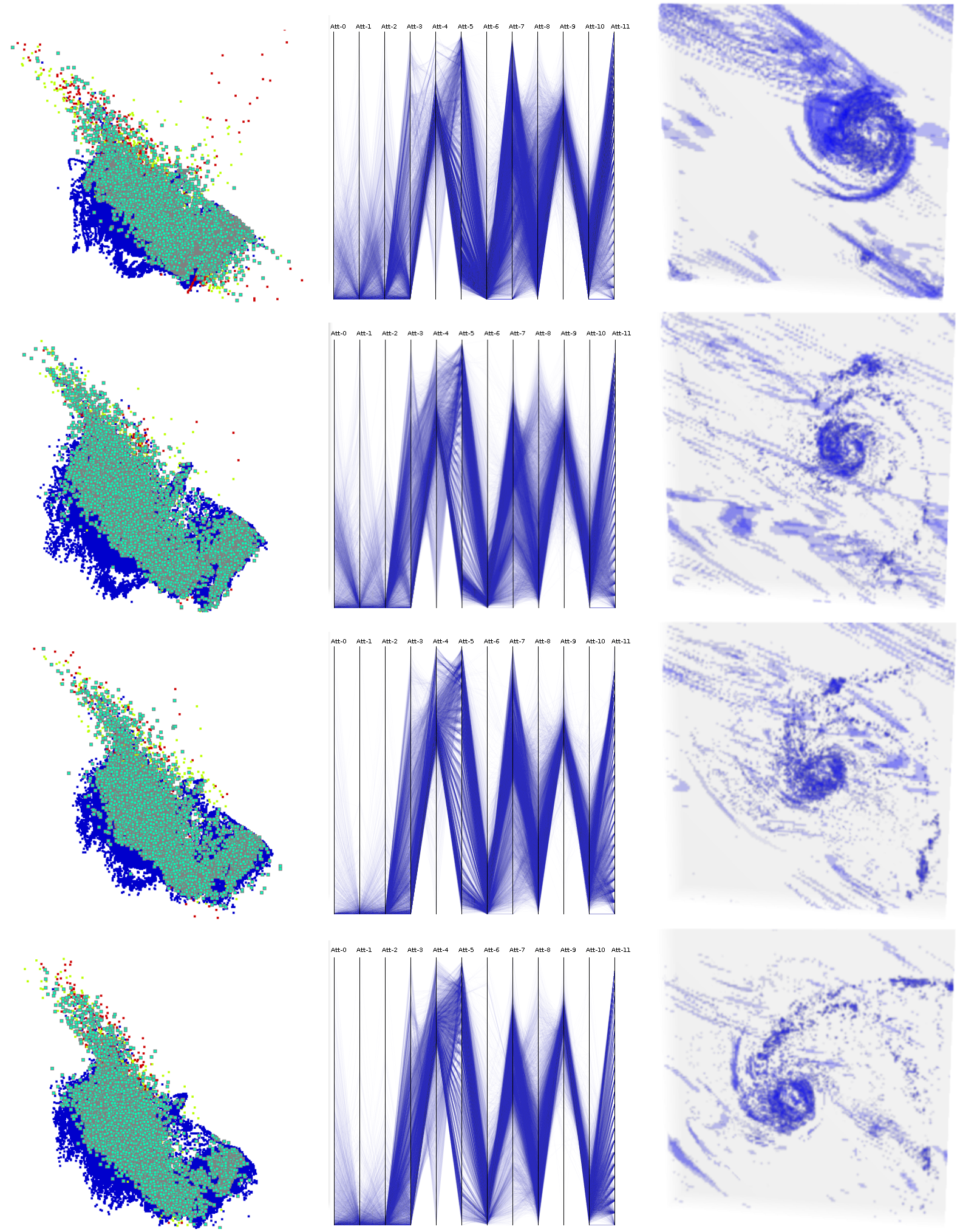

Figura 4.4: Na coluna à esquerda tem-se as projeções em quatro instantes de tempo $(05,15$, 25 e 35). Os pontos destacados nas projeções correspondem aos elementos da faixa B (variação entre $4 \%$ e $8 \%$ ). Na coluna do meio tem-se as Coordenadas Paralelas do agrupamento destacado. Na coluna à direita tem-se o espaço do objeto do agrupamento. 


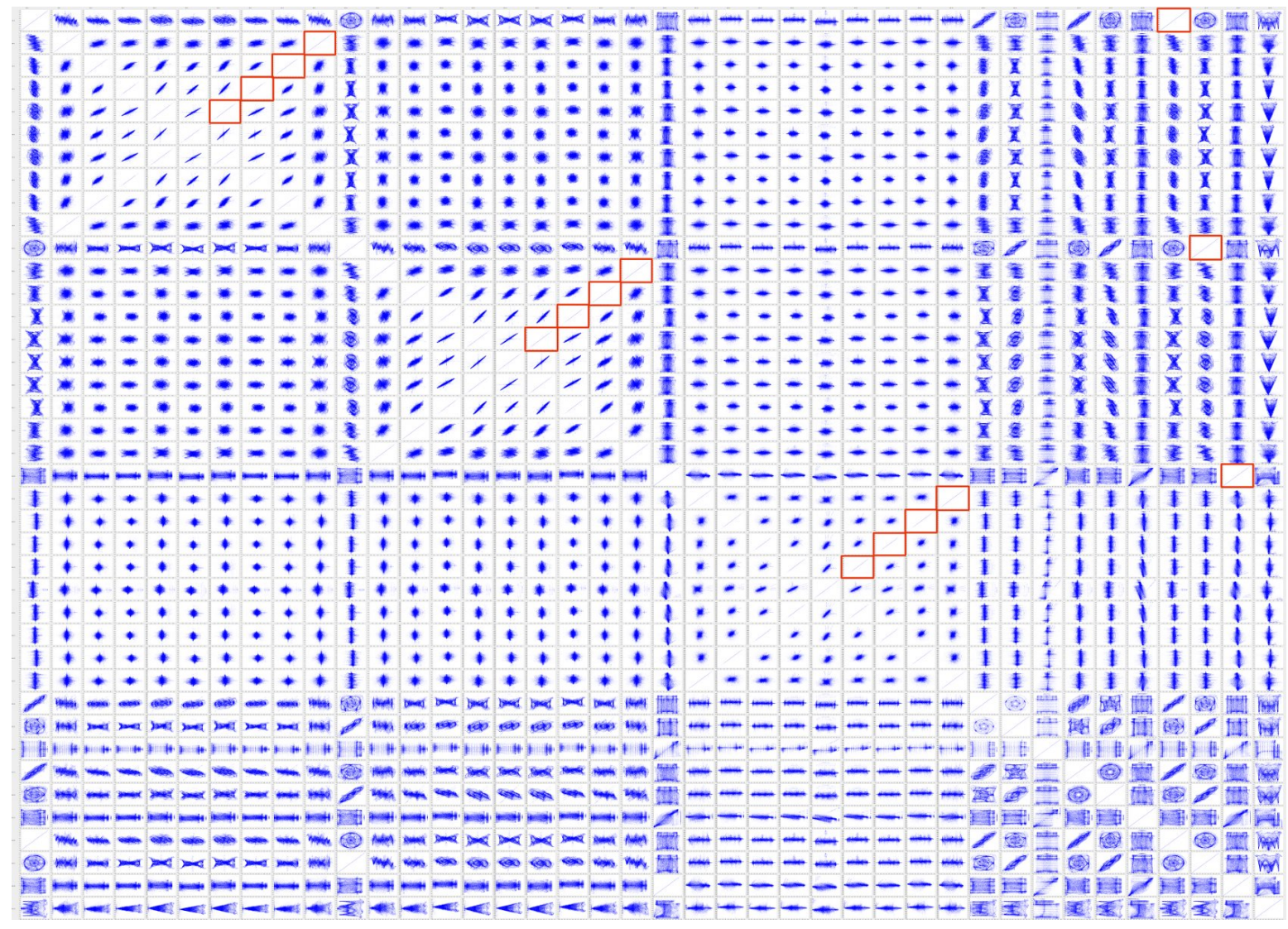

Figura 4.5: Matriz do gráfico de dispersão do conjunto de dados extraído das streamlines geradas para a simulação da bomba hidráulica (Viscontest 2011). O conjunto possui 40 atributos, gerando uma matriz 40x40. 

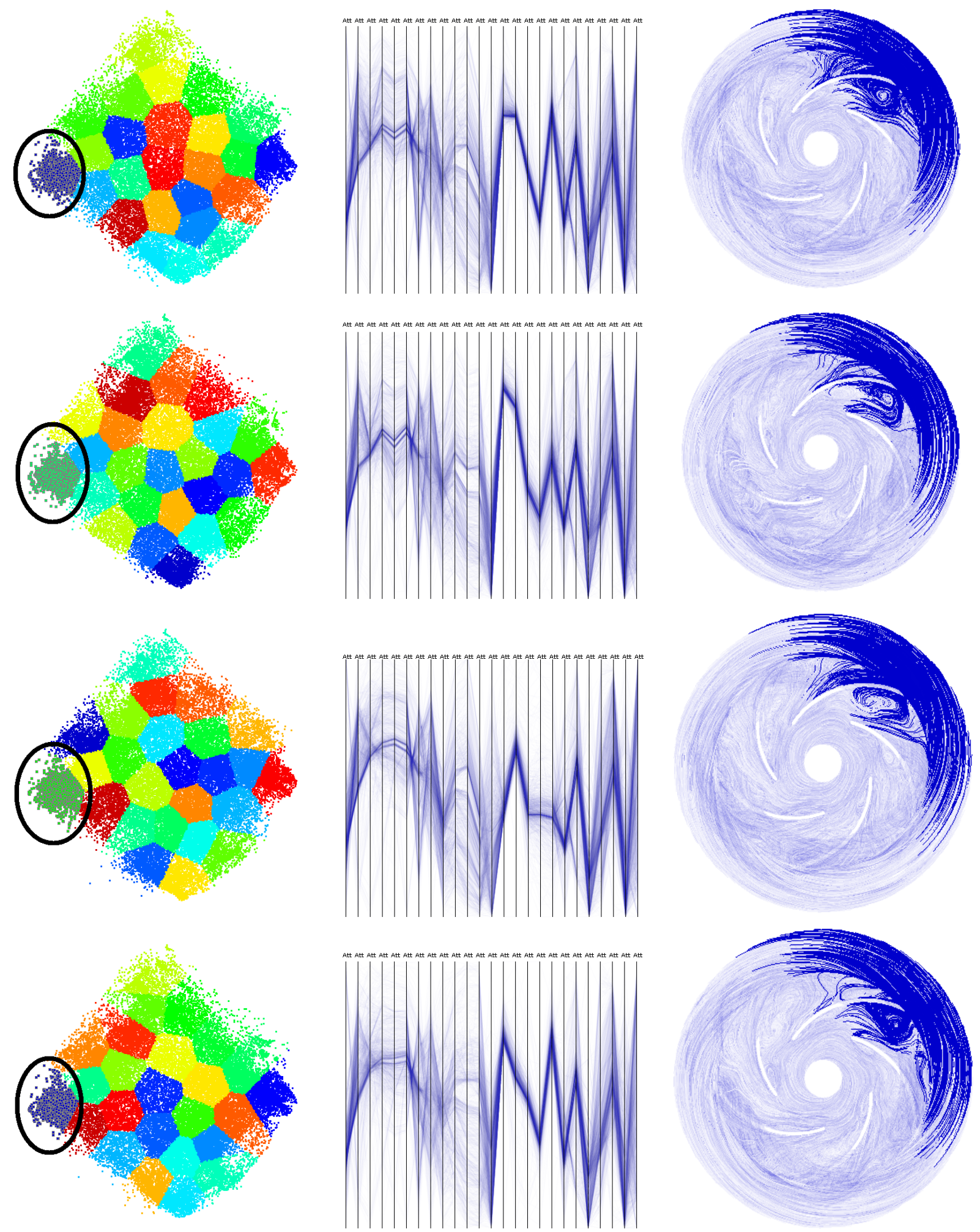

Figura 4.6: Na coluna à esquerda tem-se as projeções referentes a quatro instantes de tempo $(10,30,50$ e 70). Os pontos destacados nas projeções correspondem aos elementos de um dos agrupamentos. Na coluna do meio tem-se as Coordenadas Paralelas do agrupamento destacado. Na coluna à direita tem-se a representação do espaço do objeto (visualização das streamlines) do agrupamento destacado. 

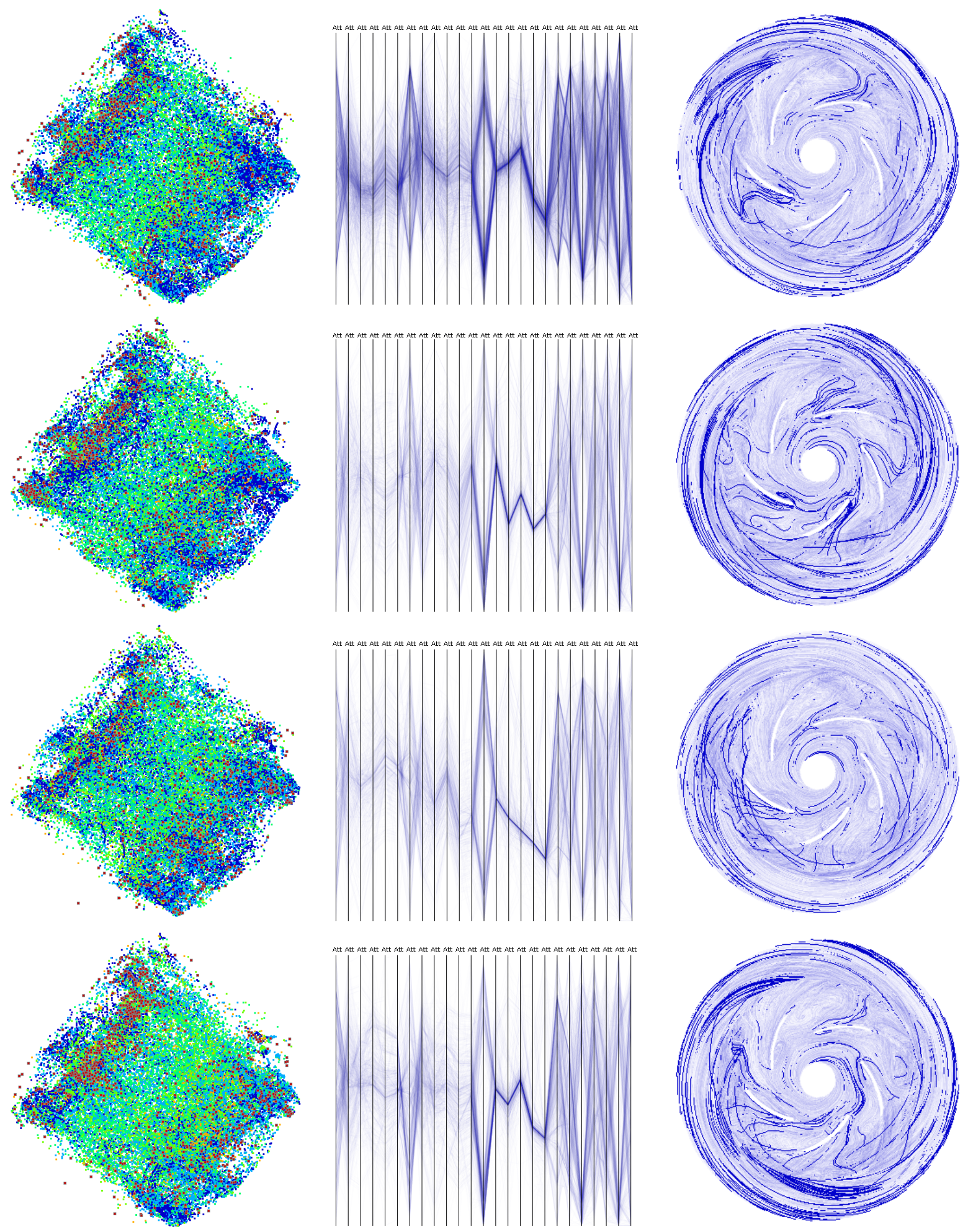

Figura 4.7: Na coluna à esquerda tem-se as projeções em quatro instantes de tempo $(11,31$, 51 e 71). Os pontos destacados nas projeções correspondem aos elementos da faixa $F$ (variação além de $25 \%$ ). Na coluna do meio tem-se as Coordenadas Paralelas do agrupamento destacado. Na coluna à direita tem-se o espaço do objeto do agrupamento. 


\section{Visualização de volumes de partículas variantes no tempo apoiada por projeções}

\subsection{Considerações iniciais}

Existe uma grande variedade de técnicas de visualização para ajudar na interpretação de um conjunto de dados em um único instante de tempo (coordenadas paralelas, matriz de gráfico de dispersão, projeção multidimensional, etc.), porém ainda são poucas as técnicas para auxiliar na análise temporal de um intervalo de tempo específico ou mesmo de toda a simulação, particularmente quando a simulação envolve múltiplas variáveis. Dessa forma, neste trabalho foi desenvolvida uma abordagem chamada de visualização de streamfeatures, que oferece uma alternativa para esse problema.

As streamfeatures condensam em uma visualização o comportamento do espaço de atributos durante um intervalo de tempo, por meio dos sucessivos posicionamentos obtidos pela aplicação em sequência de uma projeção multidimensional. O objetivo é visualizar as mudanças nas relações globais de similaridades, ocorridas ao longo da simulação, ou em um período de tempo específico. É natural, em tal estratégia, o rastreamento do comportamento da primitiva (no caso a partícula) ao longo da simulação. Muito além de acompanhar uma única partícula, essa abordagem permite, de maneira simples e rápida, acompanhar o comportamento de grupos de partículas que apresentam um comportamento semelhante, seja no espaço de atributos ou no espaço do objeto.

Para identificar os grupos de interesse no conjunto de dados, o primeiro passo é a extração das características das streamfeatures, como descrito na Seção 4.3.1. Em seguida, os vetores mul- 
50 Capítulo 5. Visualização de volumes de partículas variantes no tempo apoiada por projeções

tidimensionais resultantes são mapeados para o espaço visual, objetivando a identificação visual dos agrupamentos e tendências. A projeção também fornece um meio, simples e intuitivo, de exploração interativa dos dados investigados. Caso os grupos não estejam explicitamente visíveis (devido ao elevado número de elementos ou devido à alta sobreposição), pode-se aplicar uma técnica de agrupamento, objetivando facilitar a identificação dos grupos com comportamento semelhantes.

É importante ressaltar que essa abordagem é naturalmente aplicada às simulações de partículas. Segundo Linsen et al. (2009), Smoothed Particle Hydrodynamics (SPH) é um método completamente livre de malha para simular o fluxo de fluidos. Em vez de representar as variáveis físicas sobre uma grade fixa (como os volumes de voxels), o fluido é representado por centros de interpolação que se deslocam livremente, chamados de "partículas". Desse modo, uma partícula corresponde a uma entidade que modela o comportamento de uma amostra do fluido ao longo da simulação - ao contrário dos voxels/células, que representam uma região do domínio espacial.

Este Capítulo apresenta o processo de geração das streamfeatures (Seção 5.2) e, em seguida, a aplicação dessa abordagem a dois conjuntos de dados (Seção 5.3).

\subsection{Geração das streamfeatures}

As streamfeatures precisam ser geradas com uma técnica de projeção multidimensional precisa, altamente escalável e, principalmente, capaz de fazer o mapeamento, do espaço multidimensional para o espaço visual mantendo a coerência espaço-temporal. Tanto a PLMP como a LAMP (Seções 2.4 .5 e 2.4.7) satisfazem tais requisitos. Após a definição da técnica de PM a ser utilizada, ela é aplicada à sequência de volumes que descreve a simulação. Guardando-se o posicionamento obtido em cada um desses mapeamentos, pode-se gerar uma sequência de linhas conectando os posicionamentos da partícula em cada instante da simulação, tal polilinha é chamada de streamfeature. A Figura 5.1(a) ilustra uma visualização das streamfeatures.

A fim de facilitar a interpretação das streamfeatures, são gerados os eixos que descrevem o espaço multidimensional. Os eixos correspondem ao mapeamento de cada atributo do conjunto de dados, para o espaço visual, segundo a mesma transformação aplicado nas projeções para a geração das streamfeatures. A Figura 5.1 apresenta as streamfeatures como os seus eixos.

Tanto a PLMP como a LAMP utilizam um subconjunto de elementos chamados de representativos. O posicionamento dos elementos representativos constitue um estágio vital para a qualidade final da projeção. Dentre as muitas opções possíveis, buscamos investigar qual poderia gerar a melhor projeção com base nos critérios de qualidade de projeção apresentados na Seção 2.5. Consideramos duas medidas para aproximar o cálculo de dissimilaridade: Euclidiana e Mahalanobis. Uma vez que os conjuntos de dados podem conter atributos correlacionados, a distância Mahalanobis constitui uma alternativa mais adequada.

A Figura 5.2 ilustra o processo de geração das streamfeatures, incluindo a investigação para identificar a melhor projeção dos elementos representativos. Dessa forma, a geração da 


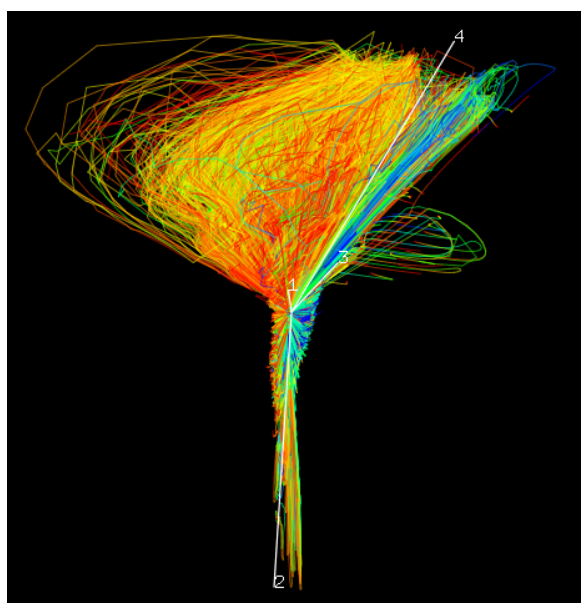

(a) Visualização das Streamfeatures.

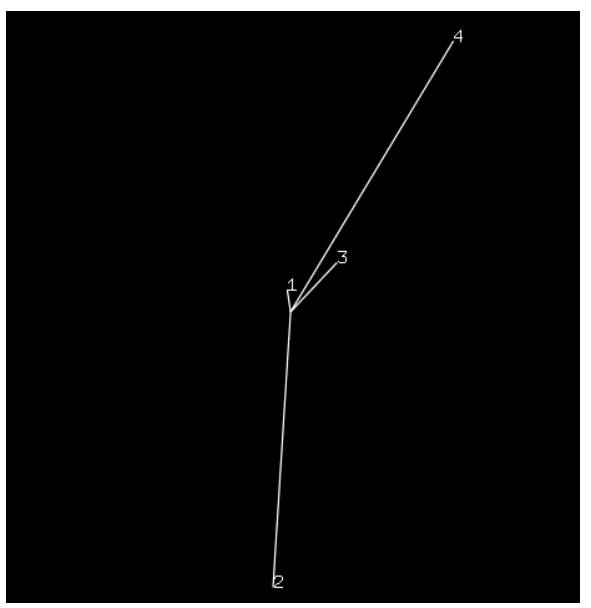

(b) Eixos no espaço das streamfeatures.

Figura 5.1: Streamfeatures (a) e os eixos 4-dimensionais (b) da simulação mini dump.

streamfeature é composta por três estágios. Primeiro, forma-se o conjunto com 5.000 elementos chamados de representativos, construído pela seleção aleatória de elementos de cada volume do conjunto de dados. Segundo, projeta-se os elementos representativos, nessa fase, os representativos são projetados com diversas técnicas de PM. Em seguida, a melhor projeção é selecionada, considerando as medidas de estresse, curvas de estresse e preservação da vizinhança (Seção 2.5). No terceiro e último estágio, aplica-se a PLMP ou a LAMP para projetar a sequência de volumes e formar a polilinha da streamfeature.

Para projetar os elementos representativos consideramos 15 métodos, vale relembrar que as técnicas Sammon's Mapping (SM) e Force Scheme (FS) iniciam seu cômputo a partir de um layout inicial, então elas são utilizadas em combinação com outras técnicas: Fastmap, Fastmap com FS, Fastmap com SM, PCA, PCA com FS, PCA com SM, LAMP, LAMP com FS, LAMP com SM, PLMP, PLMP com FS, PLMP com SM, LSP, LSP com FS e LSP com SM. PCA com FS e PCA com SM significa que o posicionamento inicial dos conjuntos é feito utilizando-se o PCA, e em seguida aplica-se a Force Scheme ou a Sammon's Mapping.

\subsection{Investigação via streamfeatures}

A visualização via streamfeature foi aplicada a duas simulações de partículas gentilmente cedidas por Lars Linsen e Vladimir Molchanov, do Visualization and Computer Graphics Laboratory da Jacobs University, Bremen.

As simulações referem-se a um sistema binário, sendo duas estrelas anãs-brancas que estão "presas" em um campo gravitacional, e consequentemente trocando massa entre si e com o espaço. A diferença entre as simulações consiste nos parâmetros utilizados para sua geração. Os atributos variantes são: massa das estrelas, distância entre elas, velocidade, temperatura, etc. A primeira simulação é chamada de $\boldsymbol{m i n i} \boldsymbol{d u m p}$, a segunda é a $\boldsymbol{d a t a}$ explode. 


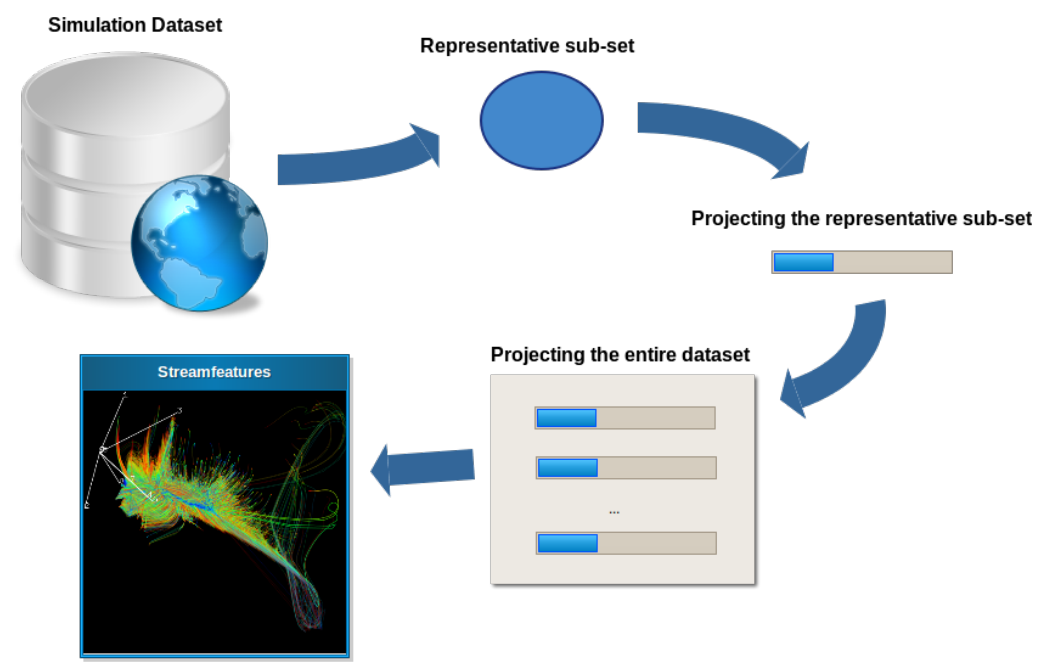

Figura 5.2: Pipeline para geração das streamfeatures.

Para a visualização das partículas no espaço do objeto, foi implementado no arcabouço desenvolvido a visualização de streampaths, também conhecida como path lines, que consiste em gerar uma poli-linha (similar as streamfeatures ou streamlines) conectando as posições espaciais da partícula, ou seja, a sua trajetória ao longo da simulação (ou de um intervalo).

\section{Mini dump}

O conjunto de dados mini dump consiste de 59 instantes de tempo, cada qual descrito por um volume com 2.517.729 partículas com quatro atributos associados: raio (1), densidade (2), temperatura (3) e av (4), como descrito na Tabela 5.1.

Tabela 5.1: Atributos da simulação mini dump.

\begin{tabular}{|l|l|l|}
\hline Id & Attribute & Description \\
\hline 1 & Raio & $\begin{array}{l}\text { Raio de influência de uma partícula. É } \\
\text { inversamente proporcional à densidade lo- } \\
\text { cal, e define quais partículas interagem com } \\
\text { a partícula considerada. Não é um valor } \\
\text { físico. }\end{array}$ \\
\hline 2 & Densidade & Valor da densidade na posição da partícula. \\
\hline 3 & Temperatura & $\begin{array}{l}\text { Valor da temperatura na posição da partí- } \\
\text { cula. }\end{array}$ \\
\hline 4 & Av & $\begin{array}{l}\text { Valor da viscosidade artificial (artificial } \\
\text { viscosity - av). Utilizado na simulação para } \\
\text { amortecer as oscilações perto da onda de } \\
\text { choque. Esta não é uma quantidade física, } \\
\text { mas um termo artificial em equações para } \\
\text { imitar a física correta. }\end{array}$ \\
\hline
\end{tabular}

Inicialmente, gera-se os layouts com as 15 técnicas de PM, considerando duas medidas de distância, para determinar como projetar os elementos representativos, conforme explicado na 
Seção 5.2. A Figura 5.3 mostra os resultados obtidos com relação a função de estresse. Observe que os três melhores resultados com a distância Euclidiana tem ordem de grandeza $10^{-5}$, obtidos com a Fastmap-FS, a LAMP-FS e a PCA-FS. Já os três melhores resultados com a distância Mahalanobis estão com a ordem de grandeza $10^{-8}$, obtidos com a Fastmap, Fastmap-FS, e a Fastmap-SM. Uma possível explicação para os resultados com a distância Mahalanobis terem sido muito superior, advém da presença da correlação entre os atributos, já que para esse caso não foi feita uma análise a procura dos atributos correlacionados, como a análise via matriz de gráficos de dispersão. Podem-se citar pelo menos 6 atributos correlacionados dos 30 extraídos das streamfeatures. Sabe-se que o primeiro coeficiente de Fourier equivale ao centro de massa, ou seja, tem-se 3 atributos de Fourier (em cada dimensão $[x, y, z]$ ) correlacionado com os 3 do centro de massa.

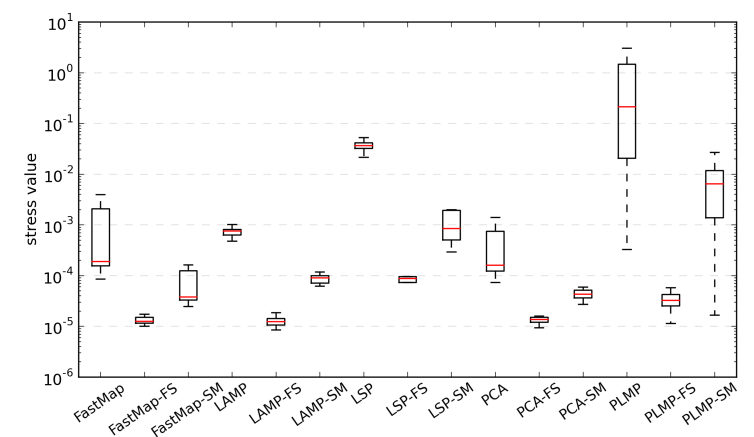

(a) Valores de estresse dos layouts computados com a distância Euclidiana

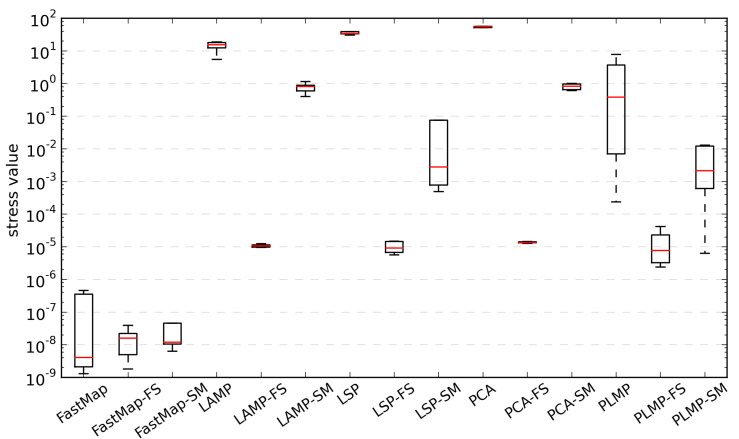

(b) Valores de estresse dos layouts computados com a distância Mahalanobis

Figura 5.3: Valores de estresse computados com 15 técnicas de projeção multidimensional da simulação mini dump.

Também foram gerados aa curvas de estresse e os gráficos de preservação da vizinhança dos três melhores resultados com a distância Euclidiana e com a distância Mahalanobis. A Figura 5.4 expõe as curvas de estresse. As três imagens inferiores apresentam melhor resultado, com uma maior concentração de pontos sobre a diagonal, e referem-se aos layouts obtidos com a distância Mahalanobis. A Figura 5.5 exibe os gráficos da preservação de vizinhança. Todos os resultados conseguiram preservar a vizinhança acima de $85 \%$.

Dessa forma, para a criação das streamfeatures selecionamos a Fastmap com FS e Mahalanobis para projetar os elementos representativos. Para projetar a sequência dos volumes foi aplicada a LAMP, sempre utilizando a distância Mahalanobis. A Figura 5.1(a) exibe as streamfeatures de toda a simulação. A Figura 5.1(b) expõe os eixos dessa transformação aplicada sobre os 4 atributos da simulação.

Foram projetadas as streamfeatures e as streampaths com a LAMP (o processo de extração de característica das linhas é aquele apresentado na Seção 4.3.1). As Figuras 5.6(a) e 5.6(b) mostram essas projeções, respectivamente, após a aplicação de uma técnica de agrupamento. As projeções apresentam uma alta sobreposição de pontos, o que dificulta a interpretação. Então, 


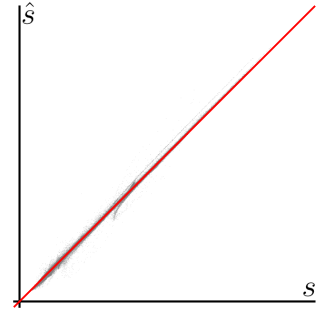

(a) Fastmap-FS-Euc

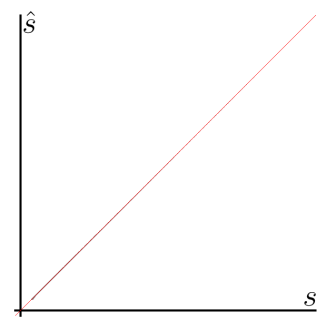

(d) Fastmap-FS-Mah

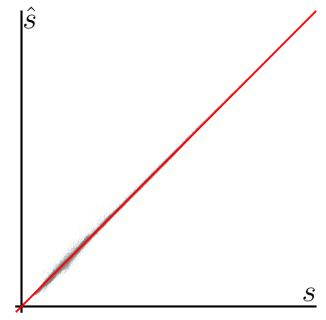

(b) LAMP-FS-Euc

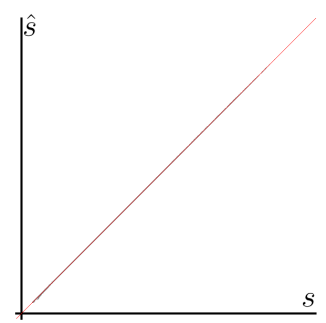

(e) Fastmap-FS-Mah

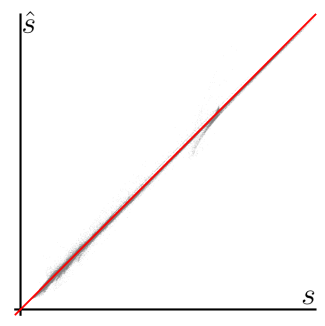

(c) PCA-FS-Euc

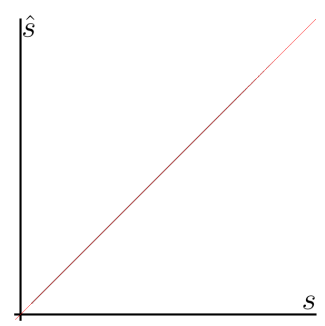

(f) Fastmap-SM-Mah

Figura 5.4: Curva de estresse dos melhores layouts dos elementos representativos da simulação mini dump: com distância Euclidiana (acima) e distância Mahalanobis (abaixo). Observa-se que as curvas de estresse dos layouts que utilizaram a distância Mahalanobis foram capazes de uma maior preservação da relação do espaço original $(s)$ pelo $\operatorname{projetado}(\hat{s})$, uma vez que a distribuição dos pontos jaz sobre a diagonal (em vermelho).

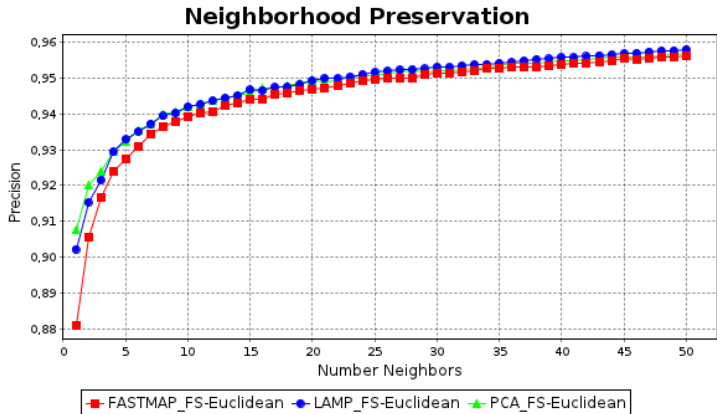

(a) Layout obtido com a distância Euclidiana

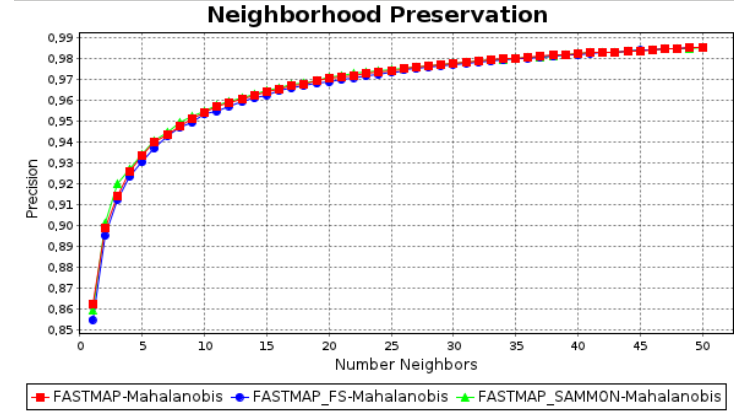

(b) Layout obtido com a distância Mahalanobis

Figura 5.5: Gráfico da preservação da vizinhança dos três melhores resultados com as distâncias Euclidiana e Mahalanobis.

sobre o layout da projeção foi aplicado o algoritmo $X$-means (com os parâmetros definidos na Tabela 5.2) e os grupos localizados refletem as cores dos pontos na figura.

A Figura 5.7 mostra a evolução das streamfeatures e as streampaths no agrupamento rosa da Figura 5.6(c). A linha superior refere-se as streampaths e a inferior as streamfeatures. Na última coluna tem-se as streamfeatures e as streampaths referentes à toda simulação (os 59 instante de tempo), porém a percepção fica prejudicada devido ao elevado número de linhas. Portanto, consideramos intervalos de tempo menores (10 instantes de tempo) e visualizamos a evolução 


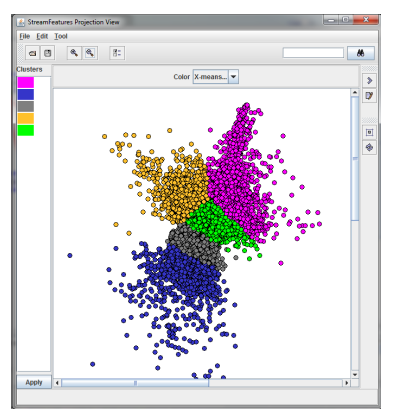

(a) Projeção das streamfeatures.

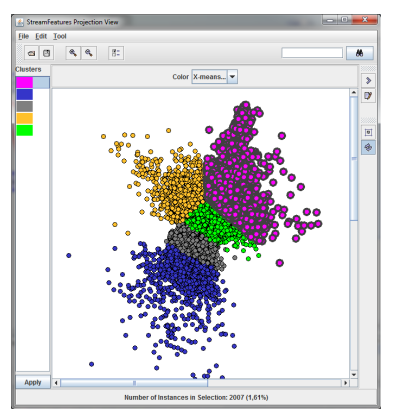

(c) Seleção de um grupo das streamfeatures.

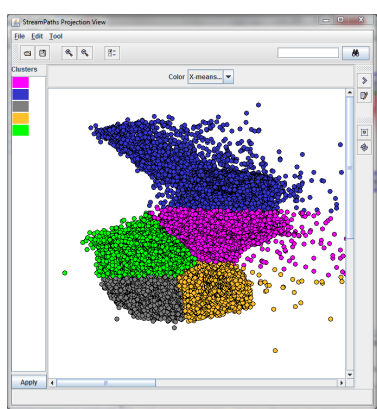

(b) Projeção das streampaths.

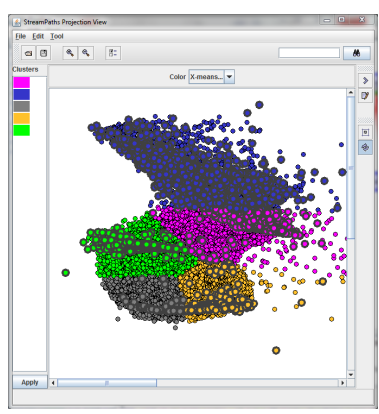

(d) Elementos selecionados na streampaths.

Figura 5.6: Agrupamentos do $X$-means nas projeções. O agrupamento rosa foi selecionado na projeção das streamfeatures (c) e os elementos correspondentes foram destacados na projeção das streampaths (d).

Tabela 5.2: Aplicação do $X$-means sobre as projeções das streamfeatures e streampaths. $\mathrm{O}$ intervalo utilizado foi [5-50].

\begin{tabular}{|c|c|c|c|}
\hline Polylines & Distance & \# Clusters & Silhouette \\
\hline Streamfeatures & Euclidean & 5 & 0.784728 \\
Streamfeatures & Mahalanobis & 5 & $\mathbf{0 . 7 9 8 6 1 0}$ \\
\hline Streampaths & Euclidean & 5 & 0.397086 \\
Streampaths & Mahalanobis & 5 & $\mathbf{0 . 4 1 5 9 6 7}$ \\
\hline
\end{tabular}

das partículas nos intervalos de 0-10, 10-20, 20-30, 30-40, 40-50 e 50-59. As cores nas imagens refletem a progressão temporal, segundo a escala apresentada na figura.

Ao observar as imagens das streampaths da Figura 5.7 percebe-se que a maioria das partículas estão se movendo para o centro das estrelas, e uma minoria se perde para o espaço. A observação das streamfeatures permite afirmar que quase não ocorreu variação na direção do eixo 2 (densidade), sugerindo que esse atributo não sofreu variação esse agrupamento durante toda a simulação. 
56 Capítulo 5. Visualização de volumes de partículas variantes no tempo apoiada por projeções

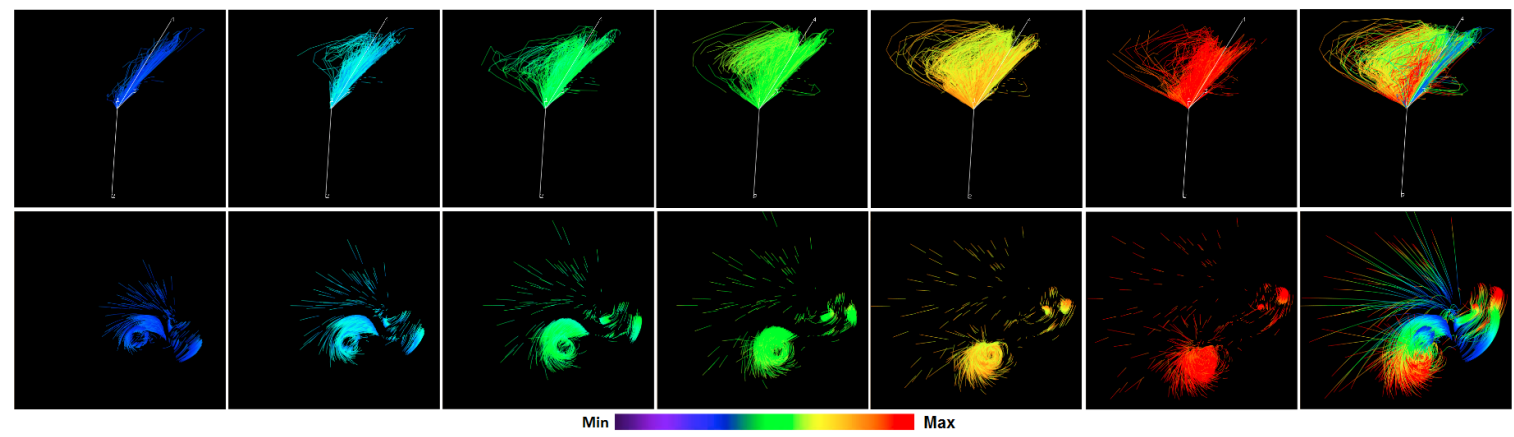

Figura 5.7: Simulação mini dump. A primeira linha corresponde a visualização das streamfeatures, já a segunda linha representa a visualização das streampaths. As primeiras seis

colunas representam as visualizações em um intervalo menor (10 instante de tempo), já a última é a visualização de toda a simulação (59 instante de tempo). As cores estão mapeando o tempo, segundo a escala exibida na parte inferior da figura.

\section{Data explode}

O conjunto de dados data explode consiste de 200 instantes de tempo, sendo que a cada instante o volume é formado por 39.200 partículas com nove atributos associados, que são exibidos na Tabela 5.3.

Tabela 5.3: Atributos da simulação data explode.

\begin{tabular}{|l|l|l|}
\hline $\mathbf{N}^{\circ}$ & Attribute & Description \\
\hline 1 & Raio & $\begin{array}{l}\text { Raio de influência de uma partícula. É in- } \\
\text { versamente proporcional à densidade local, } \\
\text { e define qual das partículas interagem com } \\
\text { a partícula considerada. Não é um valor } \\
\text { físico. }\end{array}$ \\
\hline 2 & $\begin{array}{l}\text { Energia in- } \\
\text { terna }\end{array}$ & Energia interna. \\
\hline 3 & Temperatura & Temperatura na posição da partícula. \\
\hline 4 & Helium4 & Um isótopo do hélio $\left({ }_{4}^{2} \mathrm{He}\right)$. \\
\hline 5 & Carbon12 & Um isótopo do carbono $\left({ }^{12} \mathrm{C}\right)$. \\
\hline 6 & Neon20 & Um isótopo do neônio $\left({ }^{20} \mathrm{C}\right)$. \\
\hline 7 & Magnesium24 & Um isótopo do magnésio $\left({ }^{24} \mathrm{C}\right)$. \\
\hline 8 & Silicon-group & Silicon-group. \\
\hline 9 & Abar & Abar. \\
\hline
\end{tabular}

De modo semelhante ao processo aplicado ao conjunto mini dump, inicialmente analisamos a melhor opção para projetar os elementos representativos, considerando o estresse do layout. A Figura 5.8 mostra os resultados. Os três melhores resultados com a distância Euclidiana tem ordem de grandeza $10^{-3}$ (aproximadamente), obtidos com a Fastmap-FS, LSP-FS e a PLMP-FS, e os três melhores resultados com a distância Mahalanobis tem ordem de grandeza $10^{-5}$, obtidos com a Fastmap-FS, a LAMP-FS e a PCA-FS. Mais uma vez a presença dos atributos correlacionados pode explicar o menor resultado da distância Mahalanobis. 


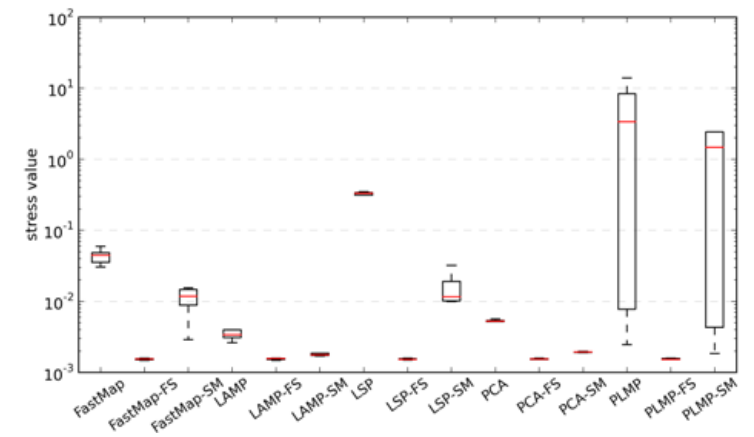

(a) Valores de estresse dos layouts computados com a distância Euclidiana

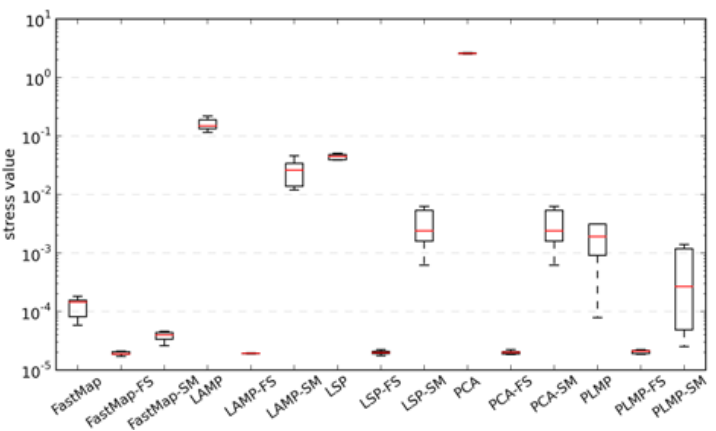

(b) Valores de estresse dos layouts computados com a distância Mahalanobis

Figura 5.8: Valores de estresse computado com 15 técnicas de projeção multidimensional da simulação data explode.

Geramos também as curvas de estresse e os gráficos de preservação da vizinhança dos três melhores resultados com ambas as distâncias. A Figura 5.9 expõe as curvas de estresse. Percebe-se que as três imagens inferiores apresentam melhores resultados, obtidos com a distância Mahalanobis. A Figura 5.10 exibe os gráficos da preservação de vizinhança. Apenas as técnicas usando a distância Mahalanobis conseguiram preservar a vizinhança acima de $67 \%$.

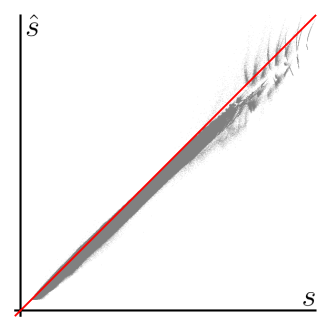

(a) Fastmap-FS-Euc

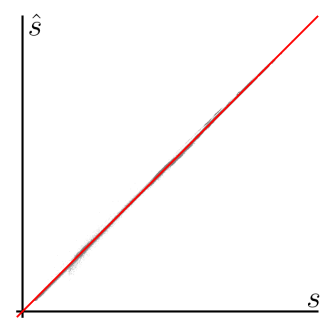

(d) Fastmap-FS-Mah

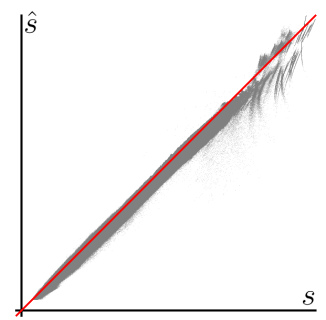

(b) LSP-FS-Euc

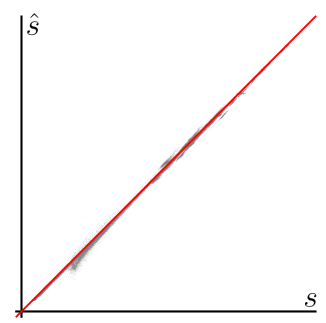

(e) Fastmap-FS-Mah

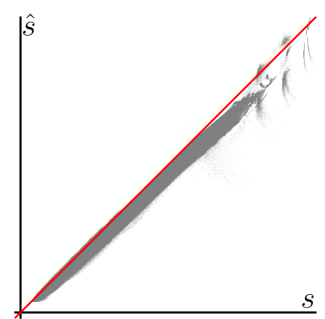

(c) PLMP-FS-Euc

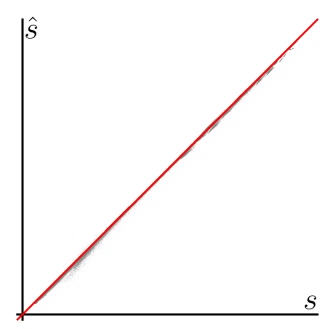

(f) Fastmap-SM-Mah

Figura 5.9: Curvas de estresse dos melhores layouts dos elementos representativos da simulação data explode: com distância Euclidiana (acima) e distância Mahalanobis (abaixo).

As streamfeatures foram geradas utilizando a Fastmap com FS para projetar os elementos representativos, e a LAMP para projeção da sequência dos volumes, com a distância Mahalanobis. A Figura 5.11(a) exibe as streamfeatures de toda a simulação. A Figura 5.11(b) mostra os eixos dessa transformação aplicada sobre os 9 atributos da simulação. 
58 Capítulo 5. Visualização de volumes de partículas variantes no tempo apoiada por projeções

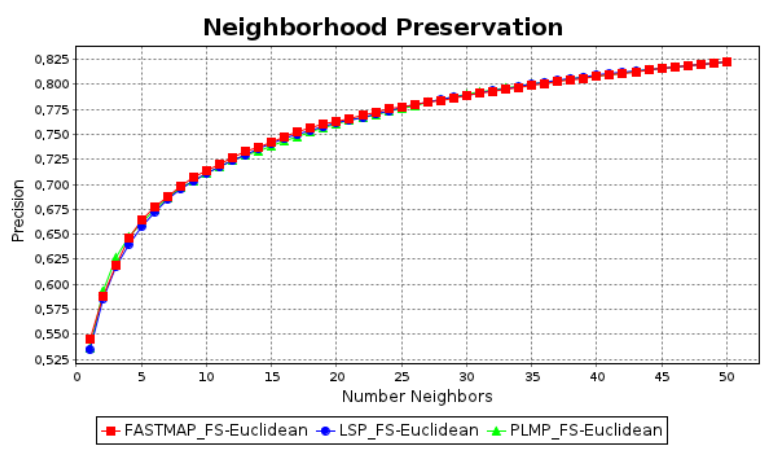

(a) Valores com a distância Euclidiana

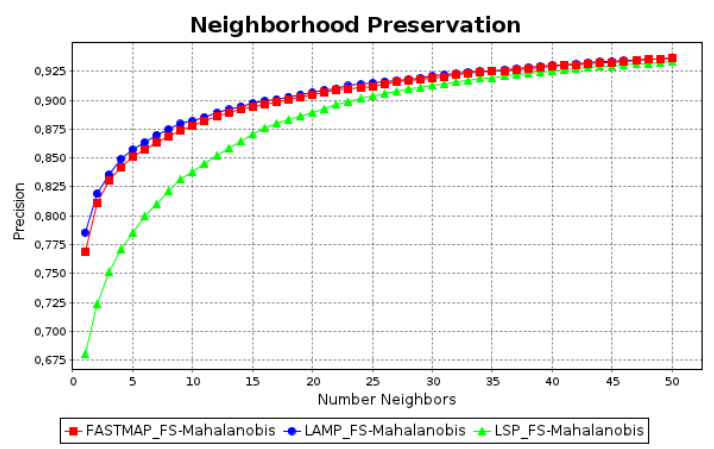

(b) Valores com a distância Mahalanobis

Figura 5.10: Gráfico da preservação da vizinhança dos três melhores resultados com as distâncias Euclidiana e Mahalanobis.

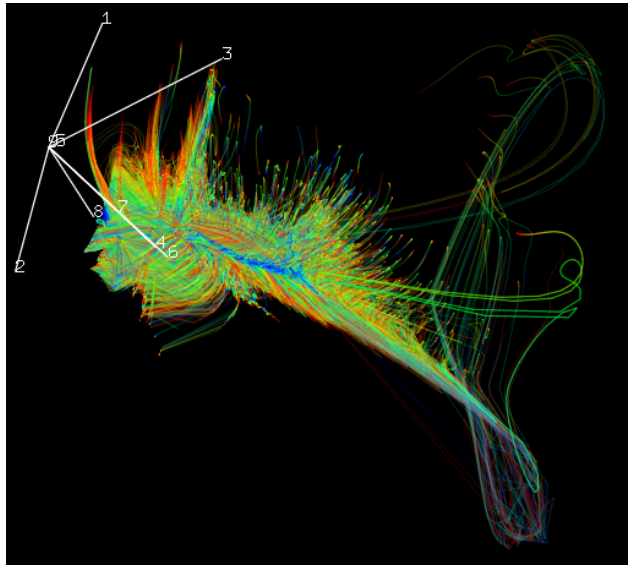

(a) Visualização das Streamfeatures.

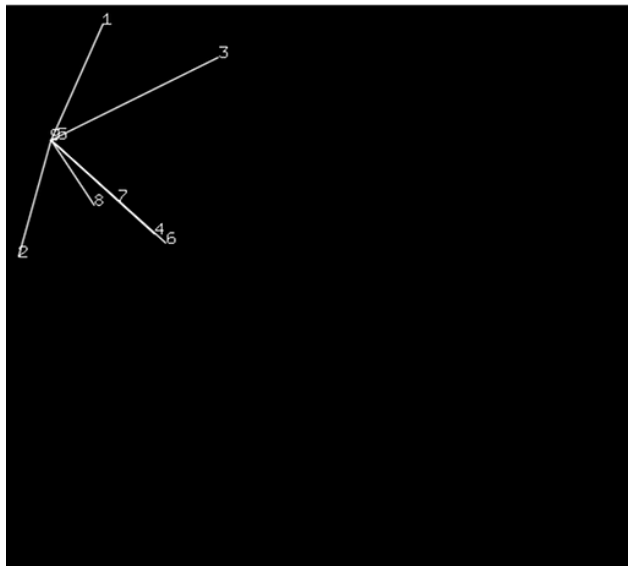

(b) Eixos no espaço das streamfeatures.

Figura 5.11: Streamfeatures (a) e os eixos 9-dimensionais (b) da simulação data explode.

Foram projetados as streamfeatures e as streampaths utilizando também a LAMP, as Figuras 5.12(a) e 5.12(b) mostram essas projeções, respectivamente, após aplicado o X-means, com os resultados e parâmetros definidos na Tabela 5.4, e os grupos localizados são identificados pelas cores.

Tabela 5.4: Aplicação do X-means sobre as projeções das streamfeatures e streampaths. O intervalo utilizado foi [5-50].

\begin{tabular}{|c|c|c|c|}
\hline Polylines & Distance & \# Clusters & Silhouette \\
\hline Streamfeatures & Euclidean & 7 & 0.456664 \\
Streamfeatures & Mahalanobis & 7 & $\mathbf{0 . 4 8 2 3 2 7}$ \\
\hline Streampaths & Euclidean & 5 & 0.583338 \\
Streampaths & Mahalanobis & 5 & $\mathbf{0 , 5 9 5 8 9 4}$ \\
\hline
\end{tabular}

A Figura 5.13 mostra a evolução das streamfeatures e as streampaths selecionadas no agrupamento rosa da Figura 5.12(c). A linha de cima refere-se as streamfeatures, já a linha de baixo 


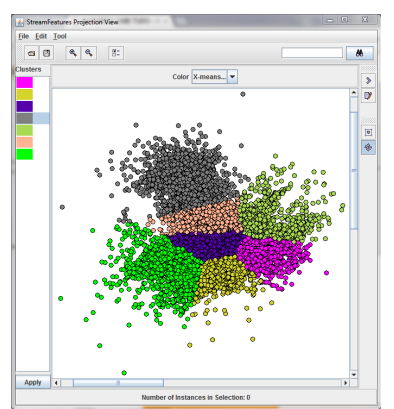

(a) Projeção das streamfeatures.

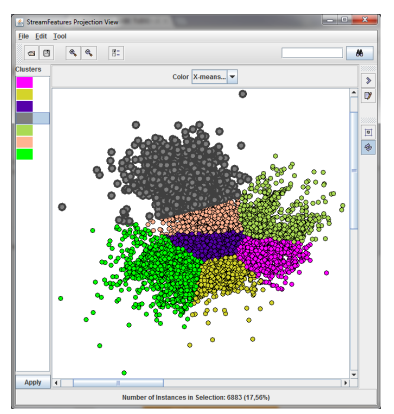

(c) Seleção de um grupo das streamfeatures.

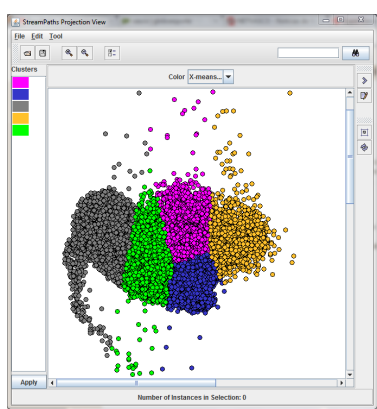

(b) Projeção das streampaths.

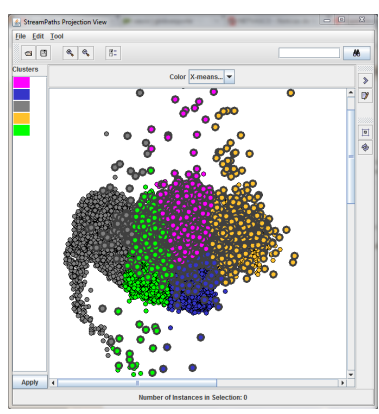

(d) Elementos selecionados na streampaths.

Figura 5.12: Agrupamentos do $X$-means nas projeções. O agrupamento rosa foi selecionado na projeção das streamfeatures (c) e os elementos correspondentes foram destacados na projeção das streampaths (d).

são as streampaths. Na última coluna tem-se as streamfeatures e as streampaths referentes a toda a simulação (os 200 instante de tempo), porém a percepção sobre essa imagem fica prejudicada devido ao elevado número de linhas. Portanto, foi aplicado um intervalo de tempo menor (20 instante de tempo) e, portanto foi visualizada a evolução das duas abordagens nos intervalos de 0-20, 40-60, 80-100, 120-140 e 140-160. As cores nas imagens referem-se à escala de cor associada ao tempo, foi utilizada a escala definida na parte inferior da Figura 5.13.

O arcabouço desenvolvido permite também filtrar as linhas que se enquadrem em certa configuração de tamanho. O usuário especifica um tamanho mínimo e um máximo, e a ferramenta filtra as linhas satisfazem tal configuração. A Figura 5.14 apresenta a interface de filtragem, neste caso foi definida sobre as streampaths. Observe que as três primeiras configurações definem streampaths, com base no seu tamanho, buscando identificar as maiores trajetórias. Já a última configuração busca identificar as menores trajetórias. A filtragem dessas quatro configurações localizou, respectivamente, $3,12,441$ e 1.888 partículas.

A Figura 5.15 apresenta as visualizações das streampaths (superior) e das streamfeatures (inferior) filtradas pelas configurações na Figura 5.14, respectivamente. Na primeira imagem (superior esquerdo), as três partículas filtradas saíram de uma estrela e foram para o espaço. Já na última imagem (superior direito) todas as 1.880 partículas correspondem ao centro de uma 
60 Capítulo 5. Visualização de volumes de partículas variantes no tempo apoiada por projeções
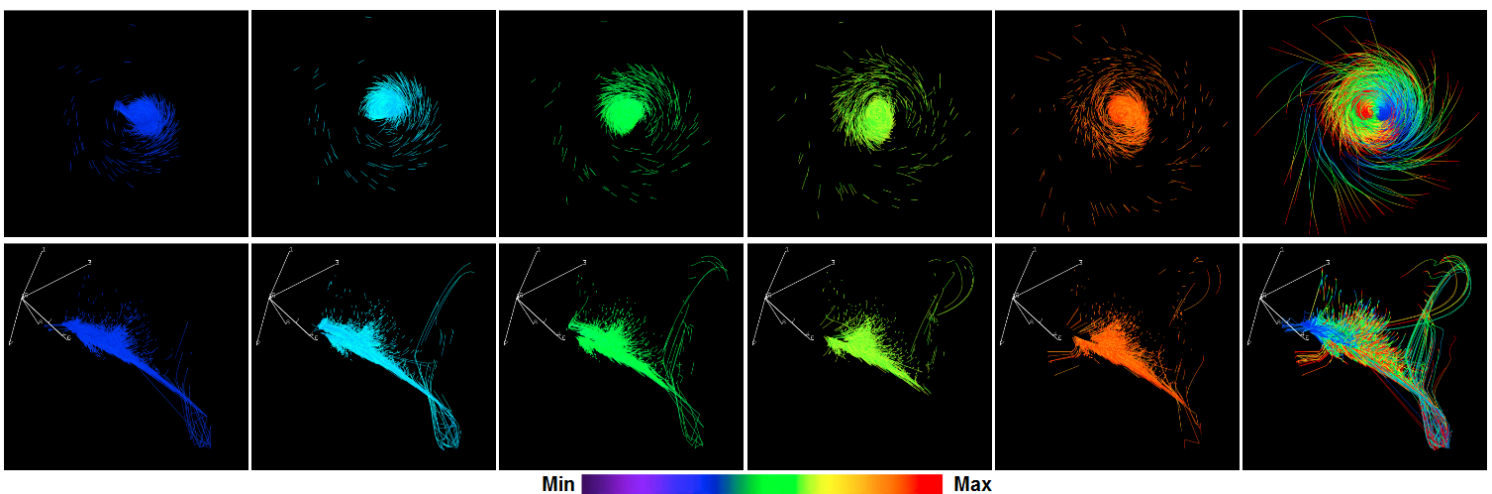

Figura 5.13: Simulação data explode. A linha superior exibe as streampaths, e a linha infeior as streamfeatures. As primeiras cinco colunas representam intervalos menores 20 instantes de tempo, já a última apresenta a visualização de toda a simulação (200 instante de tempo). As cores estão mapeando o tempo, segundo a escala exibida na parte inferior da figura.

estrela e deslocam-se juntas, com uma trajetória no formato de um arco. É possível perceber que a maioria das partículas está presa no campo gravitacional das estrelas, sendo uma minoria aquelas que escapam para o espaço.

A Figura 5.16 exibe apenas as partículas exibidas na Figura 5.14(d). Essas imagens apresentam as streampaths e as streamfeatures rotacionadas e ampliadas para ilustrar a capacidade de interação da ferramenta.

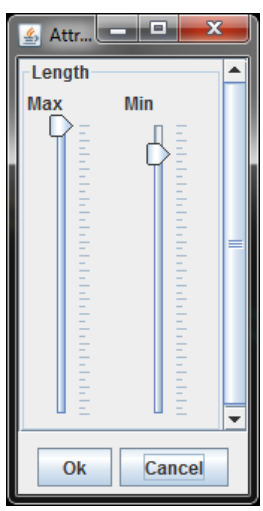

(a) 3 partículas correspondem a essa configuração.

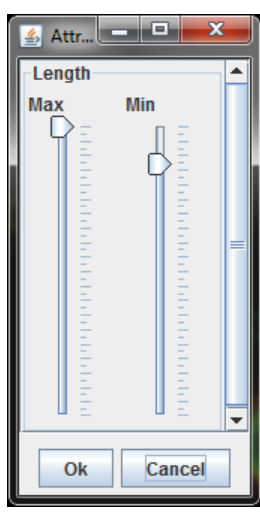

(b) 12 partículas correspondem a essa configuração.

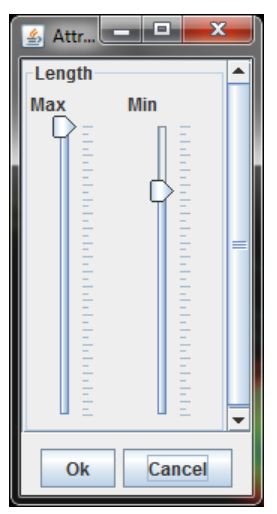

(c) 441 partículas correspondem a essa configuração.

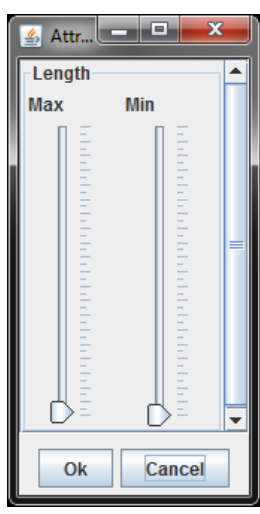

(d) 1.880 partículas correspondem a essa configuração.

Figura 5.14: Interface para a filtragem das polilinhas, neste caso das streampaths. 


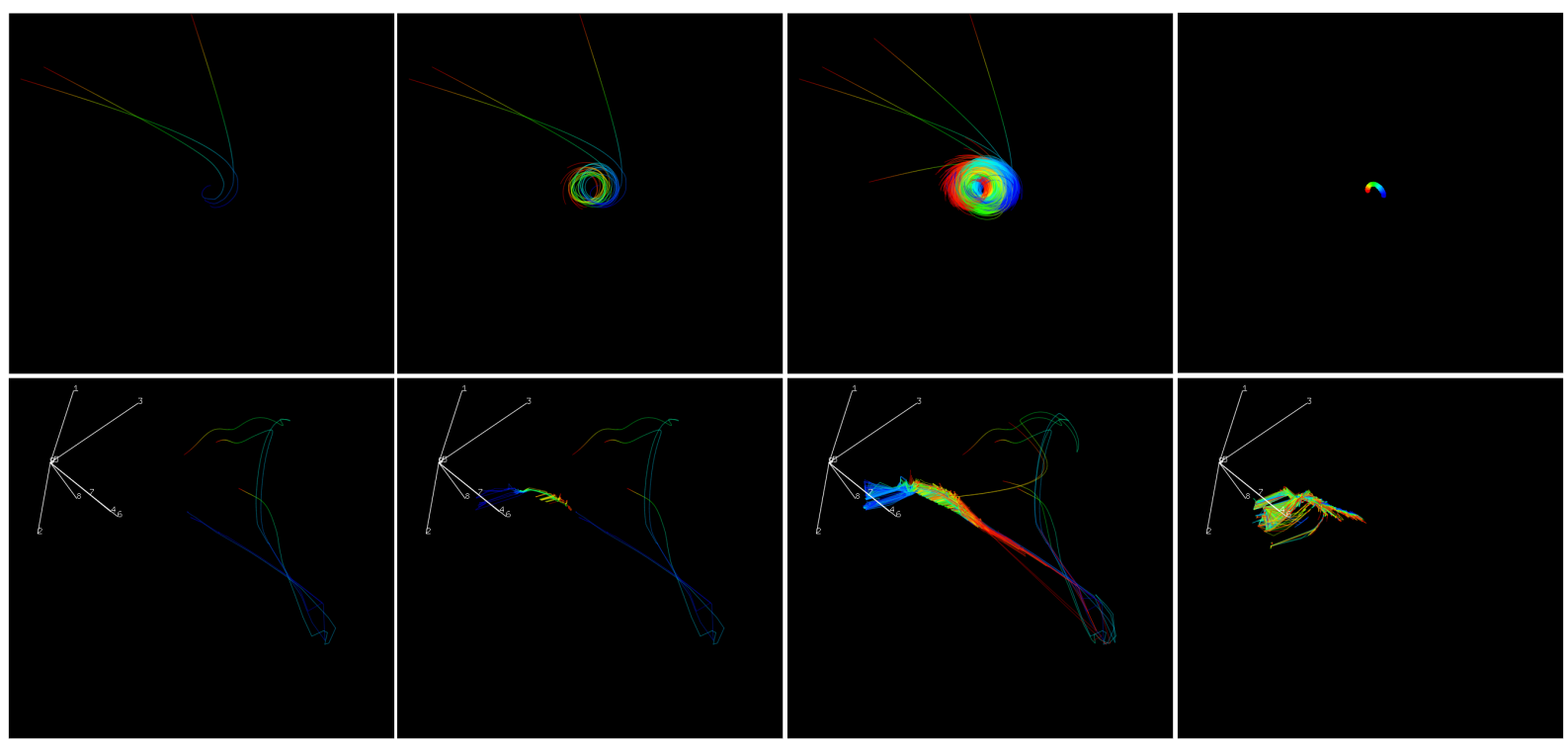

Figura 5.15: Visualização das streampaths (em cima) e das streamfeatures (em baixo) das partículas filtradas nas interfaces exibidas na Figura 5.14.

\subsection{Considerações finais}

Foi apresentada, neste Capítulo, a abordagem desenvolvida para a visualização de volumes de partículas (streamfeatures). Apresentou-se, também, a investigação via streamfeatures com duas simulações distintas. 


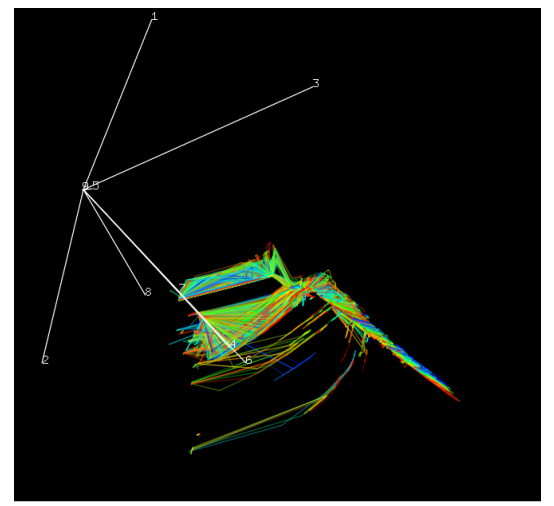

(a) Streamfeatures

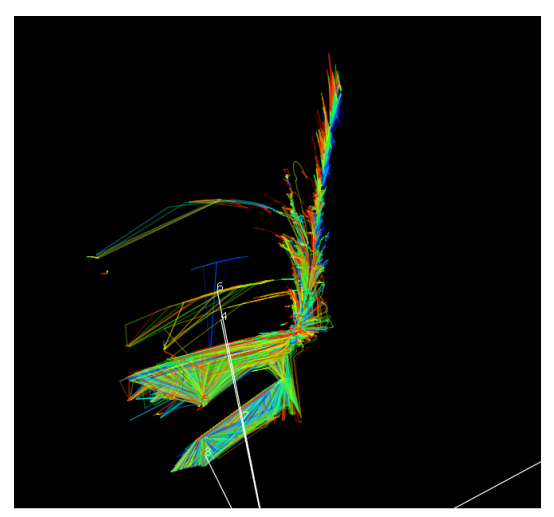

(c) Streamfeatures

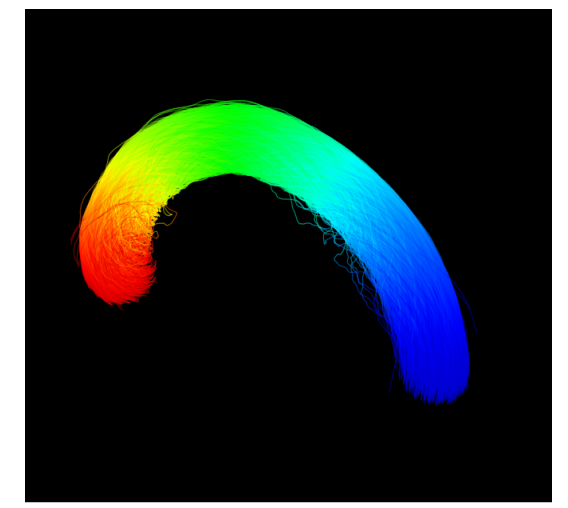

(b) Streampaths

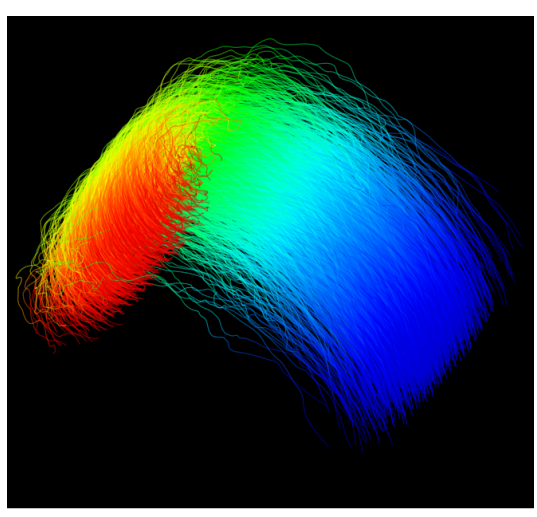

(d) Streampaths

Figura 5.16: Visualização das streamfeatures e das streampaths com as menores trajetórias, sendo um conjunto com 1.880 partículas filtradas pela interface exibida na Figura 5.14(d) 


\subsection{Contribuições}

A simulação por computador é uma ferramenta essencial para pesquisadores em diversas áreas, que por meio dela podem observar o comportamento dos mais variados fenômenos, naturais ou não. Essas simulações, principalmente quando associadas a fenômenos físicos, geram sequências temporais de volumes de diferentes tipos: volumes regulares de voxels associados a múltiplos campos escalares e vetoriais, volumes irregulares também descrevendo múltiplos campos, ou volumes de partículas. Em todos esses casos, é comum ter dezenas de gigabytes ou mesmo terabytes de dados, cuja interpretação e entendimento representa um grande desafio. $\mathrm{O}$ problema torna-se ainda mais complexo quando se considera que o fenômeno em estudo pode envolver múltiplas variáveis, e que informações importantes estão associadas não apenas ao domínio espacial, mas também ao comportamento temporal do espaço de atributos.

Este trabalho abordou este problema, investigando alternativas para apoiar a visualização exploratória de sequências temporais de volumes multivalorados, ou seja, em que cada elemento espacial tem múltiplos atributos associados. As estratégias consideradas basearam-se no uso de projeções multidimensionais para apoiar a visualização do espaço multidimensional de atributos associado à simulação, ao longo do tempo, associadas a representações visuais do domínio físico (espacial). As projeções multidimensionais buscam preservar as relações de (dis)similaridade entre elementos descritos por um vetor multidimensional de atributos. A partir de uma projeção dos dados no espaço visual, um usuário pode tentar identificar agrupamentos, tendências e correlações. Entretanto, no caso de dados volumétricos e temporais, muitas dificuldades práticas 
precisam ser consideradas - sendo que este trabalho representa uma tentativa inicial de abordar algumas delas.

Um dos problemas é que o layout da projeção fica superlotado e/ou com alta sobreposição, dificultando a interação e a percepção dos grupos. Foram implementadas estratégias eficientes de detecção automática de agrupamentos (sobre o espaço multidimensional dos dados, ou sobre o espaço projetado) para ajudar a identificar e rastrear o comportamento de diferentes grupos ao longo do tempo, utilizando a visualização dada pela projeção como meio de interação e exploração. Buscando favorecer a percepção das variações ocorridas no domínio temporal, foi implementada uma estratégia que visa realçar visualmente as regiões do espaço em que ocorreram grandes variações entre as relações de similaridade no espaço de atributos, entre dois volumes sucessivos (malhas volumétricas). Esta abordagem pode ser aplicada sobre os dados no espaço projetado ou diretamente no espaço original. Em ambas as alternativas, o volume de dados é segmentado em regiões conforme o comportamento temporal do espaço de atributos.

As abordagens anteriores são limitadas em sua capacidade de apoiar uma análise temporal essencialmente porque nas malhas volumétricas (regulares ou irrregulares) não há uma entidade a ser rastreada ao longo do tempo. No caso de volumes de partículas, é possível rastrear uma partícula no domínio temporal. Para esse caso foi desenvolvida uma abordagem capaz de capturar o comportamento temporal do espaço de atributos associados às partículas em uma única visualização, chamada de streamfeatures. Também associada a projeções, ela permite identificar partículas que apresentam comportamento semelhante (no espaço de atributos), ao longo do tempo. Adicionalmente, a visualização dos eixos temporais associados aos atributos dá uma indicação de quais predominam ao longo da simulação. Novamente, a grande quantidade de dados impõe que a análise visual seja feita sobre grupos de comportamento similar e possivelmente sobre regiões do domínio temporal, e não aos dados como um todo.

\subsection{Limitações}

Entre as limitações, pode destacar:

- A necessidade de integração dos recursos desenvolvidos com outras representações do espaço do objeto (físico), o que poderia facilitar as tarefas de análise em contextos reais.

- A ausência de uma avaliação mais sistemática e embasada das diferentes alternativas adotadas para implementar as estratégias propostas.

- A ausência de validação dessas estratégias por especialistas do domínio. O potencial foi ilustrado por meio da aplicação a conjuntos de dados representativos, mas não foi comprovado. 


\subsection{Trabalhos futuros}

Várias das limitações deste trabalho estão diretamente relacionadas às dificuldades inerentes em tratar grandes volumes de dados e prover ferramentas de análise capazes de considerar a variação no espaço temporal nesse contexto.

A aplicação de uma técnica de agrupamento temporal seria uma alternativa interessante para acompanhar a formação e evolução de agrupamentos ao longo da simulação, superando os problemas relatados na Seção 4.2.1. Entretanto, as técnicas existentes, tipicamente, apresentam custo computacional proibitivo $O\left(n^{2}\right)$ (Spiliopoulou et al., 2006) para lidar com os volumes de dados em questão. Seria necessário buscar e implementar uma solução específica para esse cenário.

Outros recursos poderiam ser incorporados ao arcabouço de análise desenvolvido, por exemplo, para mapear campos escalares na representação temporal das streamfeatures, ou para incorporar representações alternativas dos dados no domínio espacial.

Finalmente, o uso e validação das ferramentas desenvolvidas por usuários especialistas nos domínios descrito pelas simulações seria determinante para indicar quais as estratégias mais promissoras e funcionalidades adicionais. 


\section{Referências Bibliográficas}

Avila, L.; Kitware, I. The VTK User's Guide: Updated for VTK Version $5 . \quad$ Kitware Incorporated, 2006.

Disponível em http://books.google.com.br/books?id=rp4gPwAACAAJ

BlaAs, J.; Bотна, C.; Post, F. Interactive visualization of multi-field medical data using linked physical and feature-space views. Proceedings of EuroVis 07, p. 123-130, 2007.

Disponível em http://graphics.tudelft.nl/publications/blaas2007.pdf

Bristow, H. Analysing X-means Clustering for Reproducibility, Validity and Effectiveness. archive.itee.uq.edu.au, 2010.

Disponível em http://archive.itee.uq.edu.au/ comp4702/material/ CaseStudyHiltonBristow2010.pdf

Burden, R. L.; Douglas Faires Análise Numérica. São Paulo, SP: Thomson, 2001.

Disponível em http://books.google.com.br/books?id=nv7K6xVD8xYC

Co, C. S.; Friedman, A.; Grote, D. P.; Vay, J.-L.; Bethel, E. W.; Joy, K. I. Interactive Methods for Exploring Particle Simulation Data. EUROGRAPHICS - IEEE VGTC Symposium on Visualization, 2005.

Disponível em http://www.vis.1bl.gov/Publications/2005/Co-EuroVis05-LBNL-55353. pdf

Daniels, J.; Anderson, E. W.; Nonato, L. G.; Silva, C. T. Interactive vector field feature identification. IEEE transactions on visualization and computer graphics, v. 16 , n. 6 , p. $1560-8,2010$.

Disponível em http://www.ncbi.nlm.nih.gov/pubmed/20975198

Doleisch, H.; Muigg, P.; Hauser, H. Interactive visual analysis of hurricane isabel with simvis. IEEE Visualization Contest, 2004.

Disponível em http://vis.computer.org/vis2004contest/vrvis/ 
Falk, M.; Grottel, S. Interactive Image-Space Volume Visualization for Dynamic Particle Simulations. Proceedings of The Annual SIGRAD, v. Vi, 2010.

Disponível em http://www.vis.uni-stuttgart.de/eng/research/pub/pub2010/ sigrad10-falk.pdf

Faloutsos, C.; Lin, K.-I. FastMap. ACM SIGMOD Record, v. 24, n. 2, p. 163-174, 1995. Disponível em http://portal.acm.org/citation.cfm?doid=568271.223812

Ferdosi, B. J.; Roerdink, J. B. Visualizing High-Dimensional Structures by Dimension Ordering and Filtering using Subspace Analysis. Computer Graphics Forum, v. 30, n. 3, p. 1121-1130, 2011.

Disponível em http://doi.wiley.com/10.1111/j.1467-8659.2011.01961.x

Gan, G.; MA, C.; Wu, J. Data Clustering: Theory, Algorithms, and Applications. 2007.

Gribble, C. P.; Stephens, A. J.; Guilkey, J. E.; Parker, S. G. Visualizing particle-based simulation datasets on the desktop. In: In British HCI Workshop on Combining Visualization and Interaction to Facilitate Scientific Exploration and Discovery, 2006.

Disponível em http://citeseerx.ist.psu.edu/viewdoc/download?doi=10.1.1.84. 557\&amp; $r$ pe=rep $1 \& a m p ;$ type $=$ pdf

Gruchalla, K.; Marbach, J. Immersive Visualization of the Hurricane Isabel Dataset. In: Proc. 15th IEEE Visualization Conf.(VIS 04), contest entry, 2004.

Disponível em http://vis.computer.org/vis2004contest/colorado/isabel_gruchalla_ marbach.pdf

Han, J.; Kamber, M. Data Mining: Concepts and Techniques. second ed. San Francisco, CA: Morgan Kaufmann, 2006.

Hand, D.; Mannila, H.; Smyth, P. Principles of Data Mining. Mit Press, 2001.

Disponível em http://books.google.com.br/books?id=SdZ-bhVhZGYC

Hsu, W.-H.; MeI, J.; Correa, C. D.; Ma, K.-L. Arts and Technology, v. 30 de Lecture Notes of the Institute for Computer Sciences, Social Informatics and Telecommunications Engineering. Berlin, Heidelberg: Springer Berlin Heidelberg, 136-147 p., 2010.

Disponível em http://www.springerlink.com/index/10.1007/978-3-642-11577-6

Jiang, M.; Shareef, N.; Zhang, C.; Crawfis, R.; Machiraju, R.; Shen, H. Visualization Fusion: Hurricane Isabel Dataset. In: Proc. 15th IEEE Visualization Conf.(VIS 04), contest entry, 2004.

Disponível em http://scholar.google. com/scholar?hl=en\&btnG=Search\&q=intitle: Visualization+Fusion:+Hurricane+Isabel+Dataset\#0 
Joia, P.; Paulovich, F. V.; Coimbra, D.; Cuminato, J. A.; Nonato, L. G. Local Affine Multidimensional Projection. IEEE transactions on visualization and computer graphics, v. 17, n. 12 , p. $2563-71,2011$.

Disponível em http://www.ncbi.nlm.nih.gov/pubmed/22034378

Jones, C.; Ma, K.-L.; Ethier, S.; Lee, W.-L. An Integrated Exploration Approach to Visualizing Multivariate Particle Data. Computing in Science \& Engineering, v. 10, n. 4, p. 20-29, 2008.

Disponível em http://ieeexplore. ieee.org/xpls/abs_all . jsp?arnumber=4548201http: //ieeexplore. ieee.org/lpdocs/epic03/wrapper.htm?arnumber $=4548201$

KAndogan, E. Visualizing multi-dimensional clusters, trends, and outliers using star coordinates. Proceedings of the seventh ACM SIGKDD international conference on Knowledge discovery and data mining - KDD '01, p. 107-116, 2001.

Disponível em http://portal.acm.org/citation.cfm?doid=502512.502530

Kashef, R.; Kamel, M. Enhanced bisecting k-means clustering using intermediate cooperation. Pattern Recognition, v. 42, n. 11, p. 2557-2569, 2009.

Disponível em http://dx.doi.org/10.1016/j.patcog.2009.03.011http://www. sciencedirect.com/science/article/pii/S003132030900096X

Kruskal, J. B. Multidimensional scaling by optimizing goodness of fit to a nonmetric hypothesis. Psychometrika, v. 29, n. 1, p. 1-27, 1964.

Disponível em http://www.springerlink.com/index/10.1007/BF02289565

Lee, B.; Yun, J.; Seo, J.; Shim, B.; Shin, Y.-G.; Kim, B. Fast high-quality volume ray-casting with virtual samplings. IEEE transactions on visualization and computer graphics, v. 16, n. 6, p. 1525-32, 2010.

Disponível em http://www.ncbi.nlm.nih.gov/pubmed/20975194

Linsen, L.; Molchanov, V.; Dobrev, P. SmoothViz: Visualization of Smoothed Particles Hydrodynamics Data. Hydrodynamics/Book, 2009.

Disponível em http://www.intechopen.com/source/pdfs/23090/InTech-Smoothviz_ visualization_of_smoothed_particles_hydrodynamics_data.pdf

Linsen, L.; Van Long, T.; Rosenthal, P.; Rosswog, S. Surface extraction from multi-field particle volume data using multi-dimensional cluster visualization. IEEE transactions on visualization and computer graphics, v. 14, n. 6, p. 1483-90, 2008.

Disponível em http://www.ncbi.nlm.nih.gov/pubmed/18989000

MA, K. Visualizing time-varying volume data. Computing in Science \& Engineering, v. 5, n. 2, p. 34-42, 2003.

Disponível em http://ieeexplore.ieee.org/xpls/abs_all.jsp?arnumber=1182960 
Manssour, I. Visualização de estruturas internas em volumes de dados multimodais. Tese de Doutorado, Universidade Federal do Rio Grande do Sul, 2002.

Disponível em http://www.lume.ufrgs.br/handle/10183/3705

Mirkin, B. Clustering for Data Mining: A Data Recovery Approach. New York, NY - USA: Chapman and Hall, 2005.

Paiva, A. C.; Seixas, R. D. B.; Gattass, M. Introdução à Visualização Volumétrica. Rio de Janeiro, RJ: Pontifícia Universidade Católica, 1-107 p., 1999.

Disponível em http://www.tecgraf.puc-rio.br/publications/artigo_1999_ introducao_visualizacao_volumetrica.pdf

Paulovich, F.; Minghim, R. Mapeamento de dados multi-dimensionais - integrando mineração e visualização. Tese de Doutorado, Universidade de São Paulo, 2008.

Disponível em http://www.teses.usp.br/teses/disponiveis/55/55134/ tde-04032009-145018/publico/tese_paulovich.pdf

Paulovich, F.; Silva, C.; Nonato, L. Two-Phase Mapping for Projecting Massive Data Sets. Visualization and Computer Graphics, IEEE Transactions on, v. 16, n. 6, p. 1281-1290, 2010 .

Disponível em http://ieeexplore.ieee.org/xpls/abs_all.jsp?arnumber=5613468

Paulovich, F. V.; Eler, D. M.; Poco, J.; Nonato, L. G. A Fast Projection Technique and its Applications to Visualization of Large Data Sets. São Carlos, SP: Relatório Técnico do Instituto de Ciências Matemáticas e de Computação - USP, 1-23 p., 2009.

Disponível em www.icmc.usp.br/〜biblio/BIBLIOTECA/rel_tec/RT_349.pdf

Pelleg, D.; Moore, A. X-means: Extending k-means with efficient estimation of the number of clusters. In: Proceedings of the Seventeenth International Conference on Machine Learning, San Francisco, 2000, p. 727-734.

Disponível em http://staff.utia.cas.cz/nagy/skola/Projekty/Classification/ 20Xmeans.pdf

Poco, J.; Eler, D. M.; Paulovich, F. V.; Minghim, R. Employing 2D Projections for Fast Visual Exploration of Large Fiber Tracking Data. Computer Graphics Forum, v. 31, n. 3pt2, p. 1075-1084, 2012.

Disponível em http://doi.wiley.com/10.1111/j.1467-8659.2012.03100.x

Reddy, B. S.; Chatterji, B. N. An FFT-based technique for translation, rotation, and scale-invariant image registration. IEEE transactions on image processing : a publication of the IEEE Signal Processing Society, v. 5, n. 8, p. 1266-71, 1996.

Disponível em http://www.ncbi.nlm.nih.gov/pubmed/18285214

Sammon, J. A Nonlinear Mapping for Data Structure Analysis. IEEE Transactions on Computers, v. C-18, n. 5, p. 401-409, 1969. 
Disponível em http://ieeexplore.ieee.org/lpdocs/epic03/wrapper.htm?arnumber= 1671271

Samtaney, R.; Silver, D.; Zabusky, N.; CaO, J. Visualizing features and tracking their evolution. Computer, v. 27, n. 7, p. 20-27, 1994.

Disponível em http://ieeexplore.ieee.org/lpdocs/epic03/wrapper.htm?arnumber= 299407

Santos, T.; Cruz, C.; Oliveira, M.; Minghim, R.; Nonato, L.; Eler, D. Vortices Identification Based on Projection of Streamlines. IEEE Visualization Contest (viscontest.sdsc.edu), , n. 80, 2011.

Disponível em http://viscontest.sdsc.edu/2011/contributions/ VorticalStreamlines3_ts_cw/20VorticalStreamlines3_ts_cw.pdf

Schafhitzel, T.; Weiskopf, D. Investigating Hurricane Isabel Using Texture Advection. Proc. 15th IEEE Visualization, 2004.

Disponível em http://vis.computer.org/vis2004contest/stuttgart/TexAdvect.pdf

Schulze, J.; Forsberg, A. S. User Friendly Volume Data Set Exploration in the Cave. Proc. 15th IEEE Visualization Conf.(VIS 04), contest entry, v. D, 2004.

Disponível em http://www.calit2.net/ j jschulze/publications/Schulze2004b.pdf

Smith, L. A tutorial on principal components analysis. Cornell University, USA, 2002.

Disponível em http://www.sccg.sk/〜haladova/principal_components.pdf

Spiliopoulou, M.; Ntoutsi, I.; Theodoridis, Y.; Schult, R. MONIC - Modeling and Monitoring Cluster Transitions. In: Proceedings of the 12th ACM SIGKDD international conference on Knowledge discovery and data mining - KDD '06, New York, New York, USA: ACM Press, 2006, p. 706.

Disponível em http://portal.acm.org/citation. $\operatorname{cfm}$ ?doid=1150402.1150491

Tan, P.-n.; Steinbach, M.; Kumar, V. Introduction to Data Mining. Boston, MA: Pearson Addison Wesley, 2005.

Tejada, E.; Minghim, R.; Gustavo Nonato, L. On improved projection techniques to support visual exploration of multi-dimensional data sets. Information Visualization, v. 2, n. 4, p. 218-231, 2003.

Disponível em http://www.palgrave-journals.com/doifinder/10.1057/palgrave.ivs. 9500054http://ivi.sagepub.com/content/2/4/218. short

Telea, A. C. Data Visualization: Principles And Practice. Massachusetts, USA: CRC Press, 347-377 p., 2008.

Tzeng, F.-Y. Intelligent Feature Extraction and Tracking for Visualizing Large-Scale 4D Flow Simulations. ACM/IEEE SC 2005 Conference (SC'05), , n. c, p. 6-6, 2005. 
Disponível em http://ieeexplore.ieee.org/lpdocs/epic03/wrapper.htm?arnumber= 1559958

Wei, J.; Yu, H.; Grout, R.; Chen, J.; MA, K.-L. Visual Analysis of Particle Behaviors to Understand Combustion Simulations. IEEE Computer Graphics and Applications, v. 32, n. 1, p. 22-33, 2012.

Disponível em http://ieeexplore.ieee.org/xpls/abs_all.jsp?arnumber=6095486http: //ieeexplore. ieee.org/lpdocs/epic03/wrapper.htm?arnumber=6095486 


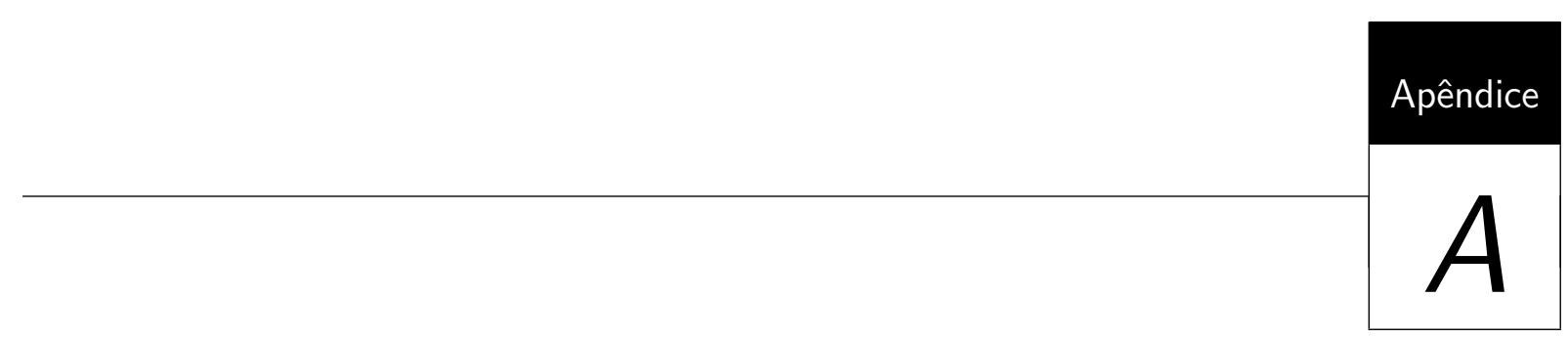

\section{Viscontest 2011}

Santos, Thiago S. Reis.; WONG, Christian C.; Oliveira, M.; Minghim, R.; Nonato, L.; Eler, D. Vortices Identification Based on Projection of Streamlines. IEEE Visualization Contest (viscontest.sdsc.edu), n. 80, 2011. 


\title{
Vortices Identification Based on Projection of Streamlines
}

\author{
Thiago S. Reis Santos* Christian Jorge Daniel Wong Cruz ${ }^{\dagger} \quad$ Maria Cristina Ferreira de Oliveira ${ }^{*}$ \\ Rosane Minghim \& Luis Gustavo Nonato ${ }^{\text {II }}$ Danilo Medeiros Eler $\|$
}

Instituto de Ciências Matemáticas e Computação - Universidade de São Paulo, Brazil

\section{INTRODUCTION}

In this work we present a visualization strategy to support identification of vortices in a vector data set. Usually, two methods may be employed for this task: lambda $a_{2}$ [3] or the $Q-$ criterion[2]. However, these methods are not necessarily the best options for vortex identification. Rather than using the original attributes and selecting either of those methods for vortex identification, we change the focus of the problem by applying a transform and conquer approach. We first compute various streamlines from the vector data and extract shape information from them by means of the Discrete Fourier Transform (DFT), building a feature space with the (low frequency) Fourier coefficients. In this way, our new problem is how to localize the vortical streamlines, i.e., the streamlines belonging to vortical regions. We do so employing multidimensional projection techniques, which strive to preserve the relationships of the high-dimensional feature space on the $2 \mathrm{D}$ projected space. The underlying idea is to locate vortical streamlines by recognizing the peculiar shapes of groups formed from projecting their distinguishing features.

We have compared different projections techniques for the task and the best results were obtained with the Piecewise Least Square Projection (P-LSP) technique [5]. Once the streamline feature space is projected, one can select groups in the $2 \mathrm{D}$ visual space and visualize the selected vortical streamlines in the object space.

The views and linking mechanisms were developed in Java and the streamline visualizations were done using The Visualization Toolkit ${ }^{1}$.

\section{Visual Exploration Process}

The data set made available by the Vis Contest 2011 organizers results from a time-varying fluid dynamics simulation of a pump. The simulation includes different attributes: pressure, total pressure, total pressure in stn frame, turbulence kinetic energy, velocity and velocity in stn frame. The simulation dataset consists of 80 time steps, each one comprising 6.7 million nodes and 6.4 million cells. Furthermore, three data sets are available from the same centrifugal pump, by the application of three distinct turbulence models: LES, RANS and the hybrid LES/RANS. We focused our efforts in the LES simulation model.

Each time step is divided in 24 parts. In this work we have focused our analysis in the rotor domain (i.e., all volume elements named with ROTOR_VOL), which consists of 2.24 million nodes.

A major problem in streamline computation regards seed positioning. We compute streamlines with seeds uniformly distributed

\footnotetext{
*e-mail: thiagors@icmc.usp.br

†e-mail: cwong@icmc.usp.br

¥e-mail: cristina@icmc.usp.br

§e-mail: rminghim@icmc.usp.br

Ile-mail: gnonato@icmc.usp.br

"e-mail: eler@icmc.usp.br
}

throughout the whole volume, using the nodes as seeds. We believe that this approach is able to cover all regions of the volume. As the streamlines tend to cover similar regions, we have used only a subset consisting of 22,478 nodes to generate the same number of streamlines.

The streamline feature space is computed by applying a Fourier transform over the 3D points on the streamline path [7]. The 1D Fourier transform is computed for the $\mathrm{X}, \mathrm{Y}$ and $\mathrm{Z}$ coordinate arrays. We then select only coefficients related to low frequencies, 20 coefficients per coordinate, resulting on a feature vector with 60 coordinate values for each streamline.

The streamline vector space is projected onto a 2D plane, using an adapted version of the PLSP technique. The adaptation introduced fixes certain control points across different time stamps to maintain visual coherence accross successive time steps. Users can locate and select in the projection well formed groups of points representing vortical streamlines. As multidimensional projections such as PLSP strive to derive a layout that preserves proximity of highly similar instances (in the multidimensional feature space), vortical streamlines are likely to group in the same projection regions over time. When a neighboring time step is loaded, the object space is updated according to the previous user selection. The nodes corresponding to selected points are then rendered in object space. Linking between the feature and object space representations provides an interaction mechanism that supresses the need of users handling three dimensional spaces or interacting with complex structures in the volume.

Figure 1 illustrates the exploration process enabled by our linked view approach. Initially, in Figure 1(a) the first time step is loaded and visualizations of both the projected feature space and the fluid are shown. Next, in Figure 1(b), the user selects a few groups in the projection (left image) which are then shown in object space (right image), revealing that the selection comprises groups of vortical streamlines. This same user selection is maintained when the next (second) time step is loaded. As illustrated in Figure 1(c), spatial coherence is preserved between the projections, that is, the vortical streamlines belong to the same selected region.

Figure 2 illustrates a vortex, highlighted by the red polygon, appearing in the sequence comprising time steps 5 to 10 . The vortex appears from the inside to the outside part of the rotor blade. Projections and selections corresponding to Figure 2 are shown in the attached pictures.

\section{Time Consumption}

The visualization application was executed on a Intel i7 $2.8 \mathrm{GHz}$ computer with $16 \mathrm{~GB}$ of RAM and an IDE hard disk. Prior to exploration the data set was preprocessed to generate the streamlines for each vertex, with a step of 100. Streamlines were computed employing the vtkStreamTracer class, and computing the $22 \mathrm{k}$ streamlines took approximately three hours. We have also preprocessed the projections associated to all (80) time steps, which takes under 18 minutes. The streamlines and the projection relative to one time step (both stored in the hard disk) are loaded during visual exploration in just under a minute. Coordination between projection and object space is done in real time. 


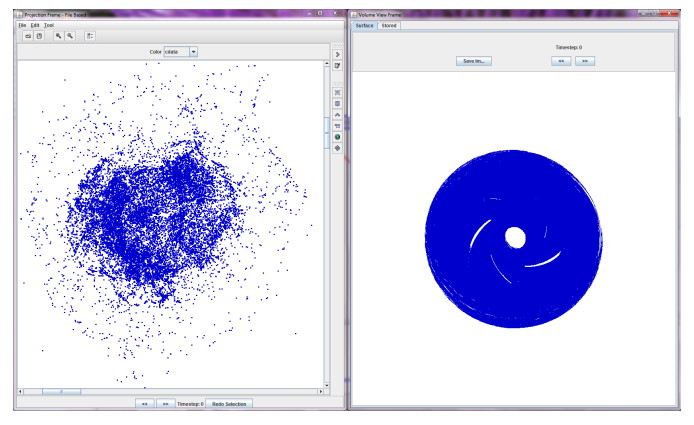

(a) Time step 0 .

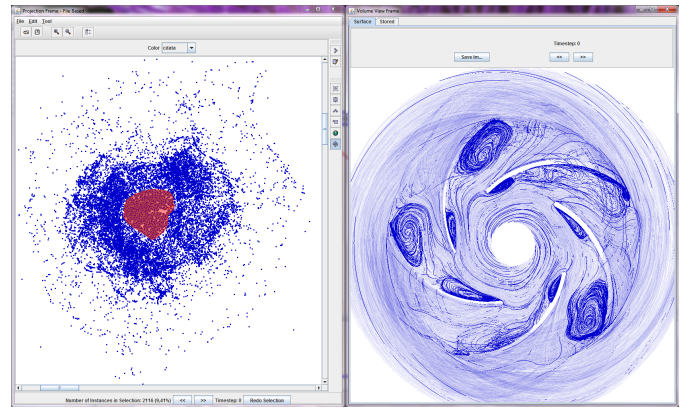

(b) Time step 0 - User selection.

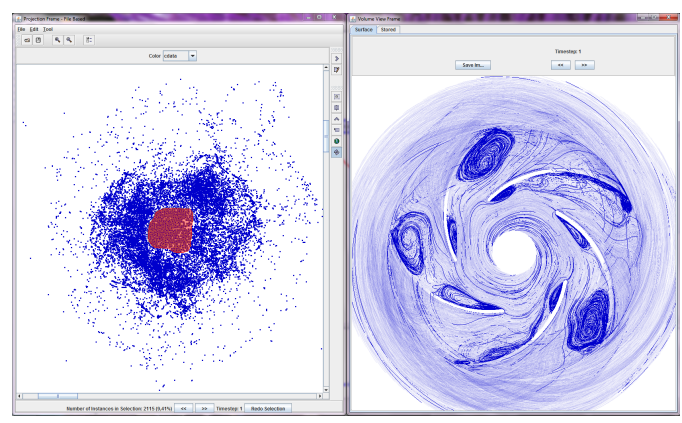

(c) Time Step 1 - Same position of previous user selection.

Figure 1: Identification of vortices: (a) visualizations of the streamline feature space and the object vector space; (b) Selection of well defined groups and visualization of the corresponding streamlines. Selected group corresponds to vortical streamlines; (c) Next time step loaded, maintaining the selection performed in (b), reveals the 2D spatial coherence of the projected streamlines.

\section{Comments AND Conclusions}

We initially tried to analyze the relationship of the nodes by projecting a feature space composed by the raw attributes given in the data set. The rationale was to check wether nodes with similar attributes lead to grouping of vortical regions. Results were not satisfactory, that is, apparently vortices are not well grouped by their raw simulated attributes. That first run took the team nearly 20 days, part time, to complete.

The approach described in this work was the best solution we found to enable user controlled vortex detection in a fluid flow data set. The final solution was achieved by the team in one week, also working part time.

We have also investigated other projection techniques to group the streamlines, e.g., Principal Component Analysis [4], Fastmap [1] and Part Linear Multidimensional Projection [6], but PLSP yielded the best results.

We developed a prototype tool for this linked view approach,
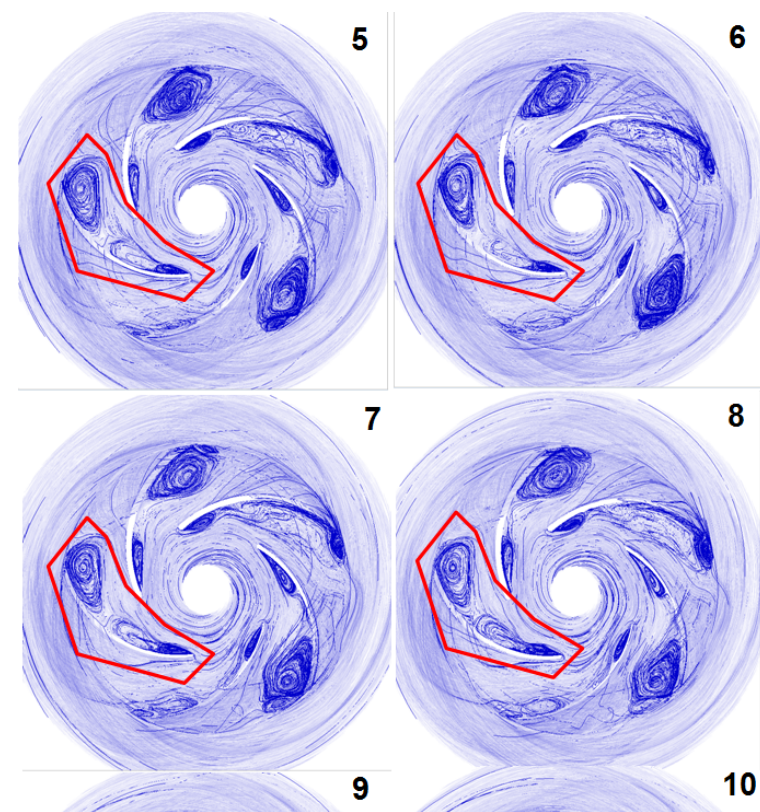

10
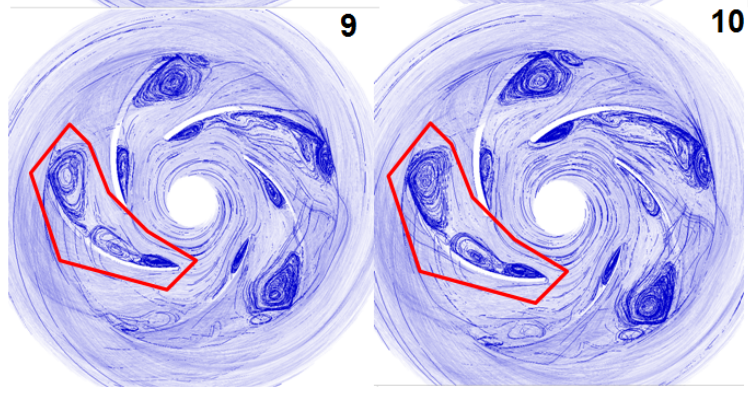

Figure 2: Illustration of a vortex appearing from the center to the border of a blade (highlighted in red).

which provides an effective way to select groups of streamlines in 2D space, aiding in the selection and identification of vortices.

\section{ACKNOWLEDGEMENTS}

This work was supported by the Brazilian research agencies FAPESP and CAPES.

\section{REFERENCES}

[1] C. Faloutsos and K.-I. Lin. Fastmap: a fast algorithm for indexing, data-mining and visualization of traditional and multimedia datasets. In Proc. 1995 ACM SIGMOD Int. Conf. Manage. of Data, SIGMOD '95, pages 163-174, New York, NY, USA, 1995. ACM.

[2] G. Haller. An objective definition of a vortex. J. of Fluid Mech., 525:1$26,2005$.

[3] J. Jeong and F. Hussain. On the identification of a vortex. J. of Fluid Mech., pages 69-94, 2851995.

[4] I. Jolliffe. Principal Component Analysis. Springer-Verlag, New York, NY, USA, 2nd edition, 2002.

[5] F. V. Paulovich, D. M. Eler, J. Poco, L. G. Nonato, C. P. Botha, and R. Minghim. A Fast Projection Technique and its Applications to Visualization of Large Dat Sets. Technical Report 349, Instituto de Ciências Matemáticas e de Computação (ICMC), Universidade de São Paulo (USP), March 2010.

[6] F. V. Paulovich, C. T. Silva, and L. G. Nonato. Two-phase mapping for projecting massive data sets. IEEE Trans. on Vis. and Comp. Graph., 16:1281-1290, 2010.

[7] M. A. Pazoti, R. E. Garcia, J. Dalton, C. Pessoa, and O. M. Bruno. Comparison of shape analysis methods for guinardia citricarpa ascospore characterization. Elect. J. of Biotech., 8(3):265-275, 2005. 


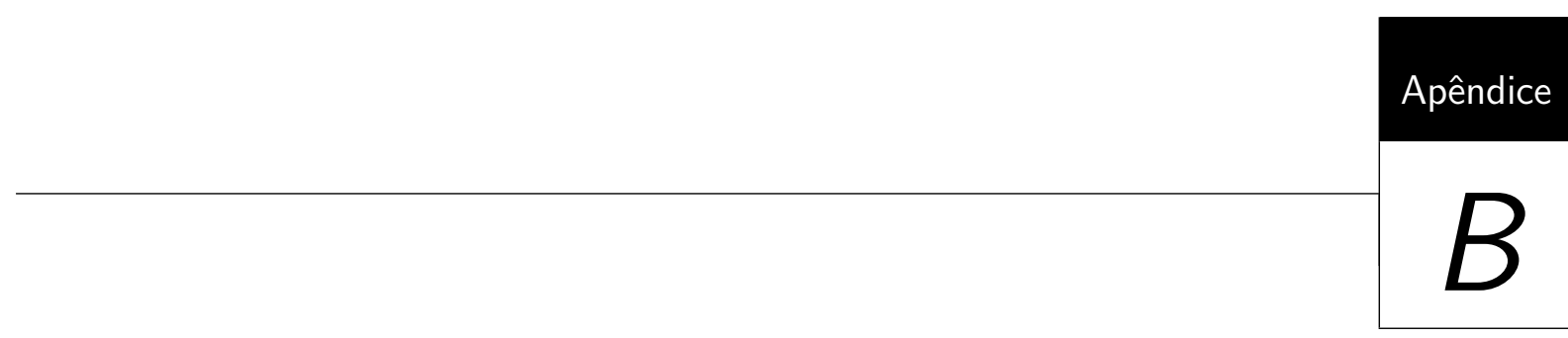

IVAPP 2013

Santos, Thiago S. Reis; Paulovich, Fernando V,; Oliveira, Maria Cristina F. Visualizing temporal behavior in particle-based simulations. Artigo sumetido ao IVAPP 2013. 


\title{
Visualizing temporal behavior in particle-based simulations
}

\author{
Thiago S. Reis Santos, Fernando V. Paulovich, Maria Cristina F. de Oliveira \\ ${ }^{1}$ Instituto de Ciências Matemáticas e de Computacão (ICMC), University of São Paulo (USP), São Carlos, Brazil \\ \{thiagors, paulovic, cristina\}@icmc.usp.br
}

Keywords: Volume-based particle visualization; time-varying visualization, multidimensional projection.

\begin{abstract}
Particle-based simulations generate very large time-varying and multi-attribute volumetric data sets. Visualizations of such volumes traditionally focus on the physical space, displaying particles as glyphs or with volume rendering techniques. In this paper we deal specifically with the issue of helping users to observe and interpret the multidimensional feature space and its temporal behavior, as a complement to existing spatial views. Our approach combines multiple visualizations to assist analysis of time-varying volumetric data generated by particle simulations. Coordinated views of both feature and physical spaces allow the observation of particle behavior over a period of time, or over the whole temporal domain, rather than describing a single simulation time step. Temporal behavior in the physical space is depicted as streampaths, i.e., path lines depicting particle spatial movement along the simulation, whereas the temporal behavior of the underlying multidimensional feature space is depicted in a so-called streamfeature visualization. Streamfeatures are lines describing changes in feature space along time. Interacting directly with these line representations is difficult. Thus, two supporting views are supplied for user interaction, which show 2D projections of both the streampaths (streampath projection view) and the streamfeatures (stramfeature projection view), obtained by projecting geometric features extracted from the lines. By linking all visualizations, users may interact with these projection views to identify and select representative clusters of lines that reflect similar behavior of particle features. We use data from two particle simulations to illustrate the framework and its potential to support analysis of global temporal behavior and relationships between multiple variables.
\end{abstract}

\section{INTRODUCTION}

Computer simulations allow the study of phenomena that would be unfeasible to observe in real life, e.g., nuclear simulations or simulations of collisions between stars. Particle-based techniques are widely employed in many domains, describing real-world phenomena as systems of discrete particles that satisfy certain laws and properties. Millions of particles may be required to accurately capture system behavior, thus generating very large data sets that are typically time-varying and multi-attribute, as multiple simulation variables describe each particle.

Assisting data analysis in this scenario is challenging, and several high-quality visualization techniques and approaches have been proposed. Most existing solutions focus on representing particles in the physical space, i.e., in the spatial domain of the simulation, at specific time steps. They usually rely on volume rendering solutions, or display particles as glyphs, and temporal behavior is observed from a sequence of such representations. In multivariate simulations, it is likely that users also want to observe the feature (or attribute) space defined by the multiple variables describing particle behavior. A multidimensional feature space may be visualized with techniques such as Parallel Coordinates (Inselberg, 1985), scatterplot matrices or multidimensional projections (Joia et al., 2011; Poco et al., 2011; Paulovich et al., 2010), which have been employed to complement spatial views in multi-attribute volume rendering (Blaas and Post, 2008; Linsen et al., 2008; Linsen et al., 2009).

In this work we focus on tools for visualizing the feature space in particle simulations, employing multidimensional projections in a framework that allows the visualization of changes in the similarity relationships in the underlying feature space. Central to this framework is a line glyph called a streamfeature, designed to convey temporal behavior of a particle and reveal meaningful changes in its defining feature space. The streamfeature line is obtained by concatenating particle projections generated for a sequence of simulation time steps. By displaying views of the streamfeatures generated for each particle, we contribute an approach to observe time-varying volumet- 
ric data sets derived from particle-based simulations in which: (i) particle behavior is observed by coordinating views of both feature and physical spaces (streamfeatures and streampaths, respectively); (ii) similarly to the well-known streamlines in fluid visualization, the streamfeature glyph conveys the global temporal behavior of each particle, as observed in its defining attribute space. Thus, both physical and feature space visualizations embed multiple simulation time steps.

This paper is organized as follows. Section 2 discusses related work on visualizing time-varying volume data generated by particle-based simulations. Section 3 provides a short background on multidimensional projection techniques used in this work. The computation of the streamfeature glyph is explained in Section 4. Its design considers several alternative projection techniques and similarity evaluation functions, using some comparison metrics to select a suitable combination of projection technique and similarity function. Section 5 describes the framework that incorporates the streamfeature visualization and describes how it links with alternative views and added functionalities for data exploration. Finally, Section 6 describes usage of the proposed framework to explore two particle simulation data sets, and Section 7 presents conclusions and discusses further work.

\section{Related work}

(Gribble et al., 2006) introduce a GPU-based approach to generate $3 \mathrm{D}$ visualizations of very large particle simulation datasets, focusing on generating 3D views on desktop computers at interactive rates. Their solution renders high quality particle glyphs using point sprite rendering and software-based acceleration techniques such as frustum and occlusion culling. It supports several data exploration functions and is sufficiently fast to handle multiple time-varying volumes - but apart from interactivity, no specific support is offered for analysis over the temporal domain.

(Falk et al., 2010) also propose an efficient solution for rendering the physical space. They implement a method of sliced ray casting with on-demand volume reconstruction that combines the ideas of texture slicing, ray casting and splatting. The density field is reconstructed from the particles in screen space at viewport resolution and at the sampling depths used by the volume ray casting. This solution requires less memory than an object-space ray casting and achieves superior image quality and comparable rendering performance.
(Co et al., 2004) are interested in interactive exploratory tools for simulation data in the domain of particle accelerator physics, seeking for solutions capable of depicting views of the feature space. Their suite of tools integrate $2 \mathrm{D}$ and $3 \mathrm{D}$ scatterplots and a representation of the particles as 3D-shaped glyphs aimed at conveying shape and trends in particle distributions affected by multiple electromagnetic fields. Animation conveys temporal behavior. Authors emphasize that the combination of familiar small multiple scatterplots for rapid multidimensional data exploration with selection and linked views to facilitate visual correlation of similarity results in an effective system for the exploratory tasks.

Recent contributions specifically address visualization of time-varying multivariate data and exploit the idea of integrating visualizations of both the physical (object) space and the feature (attribute) space. This is the case of the system by (Jones et al., 2008) to handle time-varying multivariate point-based data from gyrokinetic simulations. The feature space visualization, called variable visualization, is intended to show the relationships and trends among the multiple simulation variables and also to provide an intuitive interface for selecting data items. The object space, or physical visualization, shows a spatial representation of the particles as spherical glyphs (with point sprite rendering) at a single time step, and as illuminated pathlines along a range of time steps, with line color and opacity mapping a user selected variable. The variable view is based on an optimized implementation of Parallel Coordinates with brushing and alternative locking modes as enhanced facilities for users to specify selections in multidimensional space. All views are linked, so user selections on the Parallel Coordinates reflect in the physical and other views. The Parallel Coordinates view depicts variable relations at a single time step - for multiple time steps authors use 2D $x-y$ plots of the different variables, with the $x$-axis representing time.

Our work has many elements in common with that of (Wei et al., 2012), who propose a dual-space visualization approach for studying particle combustion simulation data. By "dual space" they refer to a combination of the 3D simulation domain in which the particles are advected, referred to as the physical space, and the attribute domain, in which the particle attributes evolve, called the phase space. The particle's spatial movement in physical space is called its trajectory, and its attribute variation in phase space is called its attribute evolution curve. The system incorporates user-driven semi-supervised learning to deal with the difficulties of handling and interacting with the large bulk of lines. The attribute evolution curves 
are clustered using a polynomial regression mixture model, and users can interact with groups to identify and categorize interesting behavior.

We also employ trajectory lines to depict particle behavior in both physical and attribute spaces, inspired by the approach adopted by (Poco et al., 2012) to handle brain fiber tracking data. Authors extract shape features from the 3D lines describing the fibers in order to generate 2D layouts which separates the lines based on similarity of their global shape. The layout provides an alternative visual interface that facilitates interaction with the fiber lines: by linking both views, bundles of lines with similar behavior may be user selected and further inspected. We generate line icons that summarize particle behavior in attribute space over time, as well as projections of such lines to help users to identify and interact with relevant groups of lines. The same approach is applicable to streamlines that describe particle behavior in physical space.

\section{Background on Multidimensional Projections}

Our framework relies on dimension reduction or multidimensional projection techniques. Let $X$ be a set of $n$ data points defined in a $d$-dimensional space, i.e., $X=\left\{x_{0}, x_{1}, \ldots, x_{n}\right\}$, with $x_{i}=\left\{x_{i_{1}}, x_{i_{2}}, \ldots, x_{i_{d}}\right\} \in \mathbb{R}^{d}$. Let $Y$ denote the projection of $X$ in a visual space $\left(\mathbb{R}^{2}\right.$ or $\left.\mathbb{R}^{3}\right)$. $s(*, *)$ is a dissimilarity function criterion between two data points in $\mathbb{R}^{d}$ and $\hat{s}(*, *)$ is their dissimilarity function criterion defined in the projected space. A multidimensional projection is a technique that obtains $Y$ from $X$, given $s(*, *)$, usually while attempting to minimize some error measure defined in terms of $s$ and $\hat{s}$.

Many approaches can be applied to project a highdimensional data set, e.g., classical dimension reduction techniques such as Principal Component Analysis (Jolliffe, 2002) or Fastmap (Faloutsos and Lin, 1995) may be employed to obtain a visual representation in this context by considering the 2 or 3 best ranked principal components, or by projecting the data points $x_{i} \in X$ in $p$ mutually orthogonal directions with $p=\{2,3\}$.

The Force Scheme (FS) (Tejada et al., 2003) projection adapts the classical Force-Directed Placement (FDP) strategy. It reduces computational cost by considering the pairwise distances between the data points, rather than computing attraction and repulsion forces on a mass-spring system. An initial layout random or computed with another projection method - is iteratively modified to separate data points pro- jected too close and bring together those placed too far apart, according to an error measure given by the difference between $s$ and $\hat{s}$, i.e., the ideal and the actual pairwise point distances.

Handling time-varying volumes requires techniques capable of handling large data sets and generating temporally coherent projections of time series data. Two recent solutions that meet such requirements are the Part-Linear Multidimensional Projection (PLMP) (Paulovich et al., 2010) and the Local Affine Multidimensional Projection (LAMP) (Joia et al., 2011).

The mathematical formulation of PLMP may be seen as a generalization of PCA. A linear transformation mapping to obtain $Y$ from $X$ is inferred from a mapping previously applied to a sub-set of representative data points. Representatives should be chosen to reflect data density and distribution. However, according to the authors the technique is robust to the choice of representatives, and taking $\sqrt{n}$ random samples is usually a reasonable choice. PLMP has complexity $O(n)$ and good precision, measured by stress values computed for layouts generated for several data sets. Moreover, because its transformation mapping may be computed from a reduced sub-set of points, PLMP is applicable as a streaming projection, as not all data points need to be known a priori.

Contrary to PLMP, which computes a global transformation mapping, the LAMP technique may be tuned to behave globally as well as locally. Again it relies on selecting and projecting a subset of samples, known as control points, from which a family of orthogonal mappings is built, one for each data point. Users can manipulate the placement of the control points to obtain a layout that satisfy their requirements. A reduced number of control points is usually sufficient to obtain good visual mappings, and LAMP is also competitive in terms of computational times, although it is slower than PLMP and Fastmap.

Deciding the best technique to project a given data set is not straightforward. Several quantitative measures exist that help to assess the quality of a given layout. Such measures usually attempt to capture to which extent a layout retains the distance distribution observed in the input data space. The wellknown Kruskal stress measure (Kruskal, 1964), given by equation 1 , attempts to quantify the information loss incurred in the projection mapping.

$$
\text { Kruskal }=\sqrt{\frac{\sum_{i<j}\left(\hat{s}\left(x_{i}, x_{j}\right)-s\left(x_{i}, x_{j}\right)\right)^{2}}{\sum_{i<j} \hat{s}\left(x_{i}, x_{j}\right)}}
$$

One may alternatively inspect a scatterplot of the original_distances $\times$ projected_distances for all data 
point pairs, known as stress curve (Joia et al., 2011) (several such curves are seen in Figure 4, Section 4). A hypothetically ideal projection that achieves $100 \%$ distance preservation would produce a stress curve with all points drawn along the main diagonal line in the scatterplot.

The Neighborhood Preservation (NP) chart (Paulovich et al., 2008), on the other hand, displays the percentage of neighboring points in the input space that remain in the same neighborhood after the projection, for a varying number of neighbors. This value is computed for each data point and averaged over all data points, resulting in a curve as shown in Figure 5.

\section{Generating Particle Streamfeatures}

A streamfeature is created for a particle by projecting its feature vectors extracted from a temporal sequence of volumes and connecting the projected points into a line. This line describes the particle's temporal evolution, as observed in its defining feature space. Projection may be performed with either PLMP or LAMP, as both have the capability of preserving temporal and spatial coherence when handling time series data. As discussed in Section 3, both techniques require selecting and projecting a representative sub-set of data points, and the quality of this initial projection affects the quality of the overall result. We thus empirically investigate multiple alternatives, taking the quality measures described in Section 3 as guides to identify the potentially best choice, given a data set.

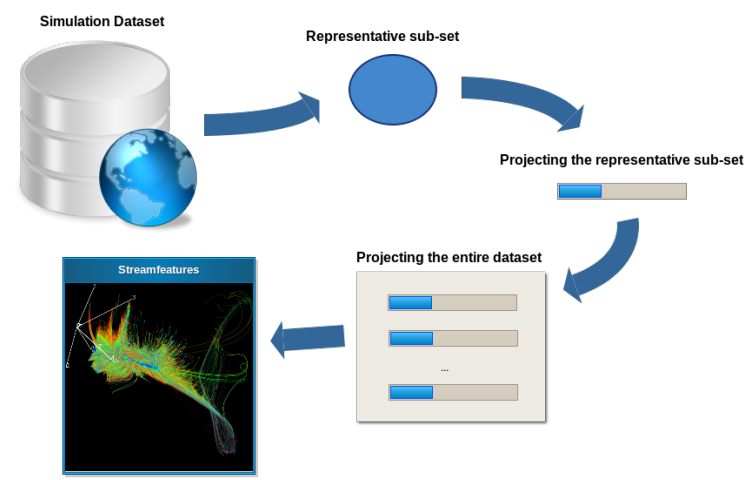

Figure 1: Pipeline of the streamfeature generation process.

Generating streamfeatures comprises three stages, illustrated in Figure 1. Initially, it may be necessary to downsample the volumes for the sequence to fit the primary memory available. This is done by random sampling. The sub-set of representative data points (a starting point required by both PLMP and LAMP, henceforth called representatives) is obtained by taking a random sample from each volume. The representatives' feature vectors must be then projected with a precise technique. Several techniques and distance functions may be considered for this initial projection, and we compare the quality of different layouts to make an informed choice.

We projected the representatives with the classical techniques PCA, FastMap, Sammon's Mapping and the Force Scheme (Tejada et al., 2003), as well as with recent ones known to be fast and precise, namely LAMP, PLMP and LSP (the Least Squares Projection) (Paulovich et al., 2008). Both Sammon's Mapping and Force gradually optimize an initial layout based on an error criterion, so alternative initial layouts may be considered. Overall we investigated 15 projection or dimension reduction techniques and variations - FastMap, FastMap with Force Scheme (meaning the former provided an initial layout for the later), Fastmap with Sammon's mapping, PCA, PCA with Force Scheme, PCA with Sammon's mapping, LAMP, LAMP with Force Scheme, LAMP with Sammon's mapping, PLMP, PLMP with Force Scheme, PLMP with Sammon's mapping, LSP, LSP with Force Scheme, LSP with Sammon's mapping - and two distance functions $s(*, *)$, Euclidean and Mahalanobis, as approximations to dissimilarity. Euclidean is a usual choice, and Mahalanobis is known to be effective in normally distributed data with multiple correlated attributes (Tan et al., 2005).

We compare the layouts obtained regarding stress function values, stress curves and neighborhood preservation capability, in order to select the best projection of the representatives for either PLMP or LAMP. In the final stage the remaining particles (the non-representatives) at each timestep are projected in $3 \mathrm{D}$, and the projected points relative to a single particle are connected into a line to obtain its streamfeature.

Figures 2 and 3 show box plots of the mean stress values computed for the layouts generated to obtain the streamfeatures for the mini dump data set (see Section 6), employing the Euclidean and the Mahalanobis distances, respectively. The Euclidean layouts with lowest stress are FastMap with Force Scheme, LAMP with Force Scheme and PCA with Force Scheme; for Mahalanobis Fastmap, FastMap with Force Scheme and FastMap with Sammon's Mapping have the lowest values. Notice that the layouts obtained using Mahalanobis have lower stress values in general.

Figure 4 shows the stress curves of the best lay- 


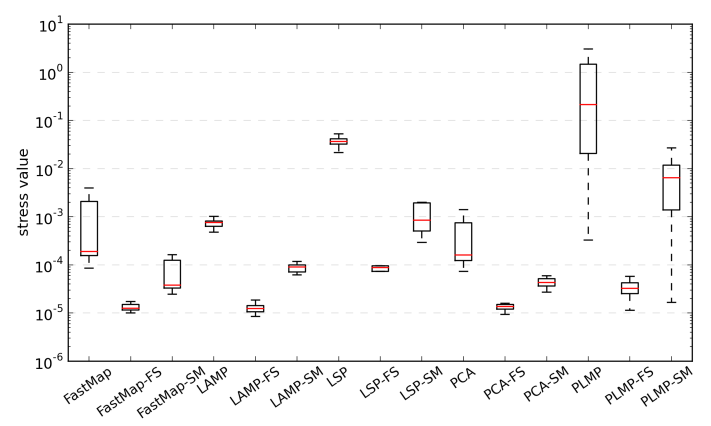

Figure 2: Kruskal stress of 15 layouts computed with distinct techniques and Euclidean distance (representatives of the mini dump data). Best: LAMP-FS, FastMap-FS and PCA-FS.

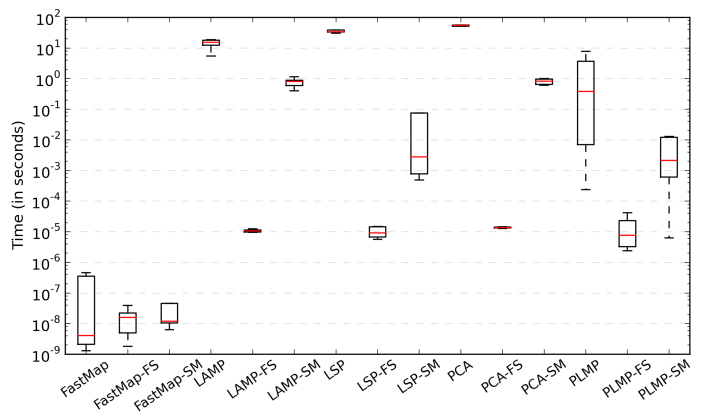

Figure 3: Kruskal stress of 15 layouts of the same data points, obtained with the same techniques and the Mahalanobis distance. Best: FastMap, FastMap-FS and FastMap-SM.

outs, considering both Euclidean and Mahalanobis distances. All do a very good job of preserving original distances, but the Mahalanobis layouts do better on this data.

Figure 5 shows the neighborhood preservation charts for the same 15 layouts, with Euclidean dis-

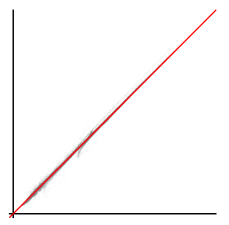

(a) Fastmap-FS (E)

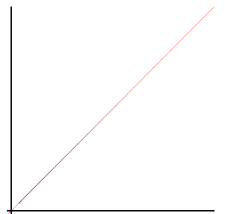

(d) Fastmap-FS (M)

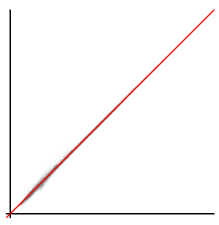

(b) LAMP-FS (E)

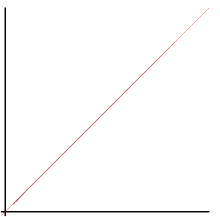

(e) Fastmap-FS (M)

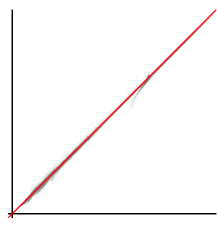

(c) PCA-FS (E)

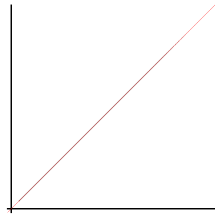

(f) Fastmap-SM (M)
Figure 4: Stress curves of the best layouts of the representatives in mini dump, obtained with Euclidean (top) and Mahalanobis (bottom) distances.

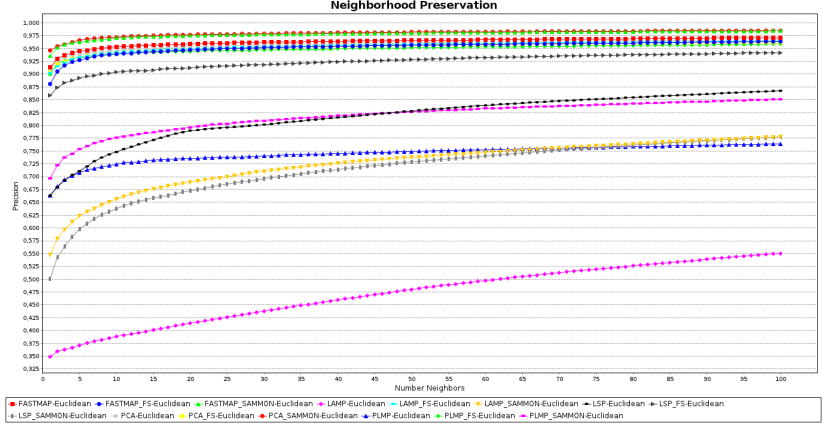

(a) NP curves for layouts computed with Euclidean. Best: PCA with Sammon's Mapping and FastMap with Sammon's Mapping.

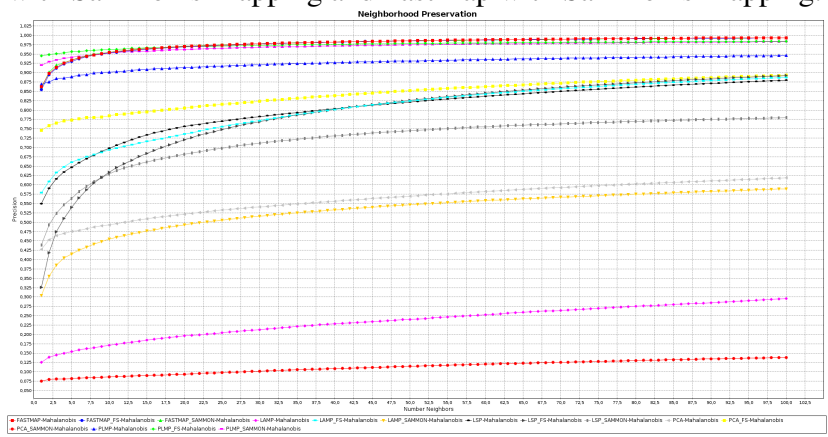

(b) NP curves for layouts computed with Mahalanobis. Best: PLMP with Force Scheme and PLMP with Sammon's Mapping.

Figure 5: Neighborhood preservation curves of the 15 layouts of the mini dump representatives.

tance (a) and with the Malahanobis distance (b). For the Euclidean layouts the three techniques with best stress values also display the best behavior regarding neighborhood preservation, and the top ones are PCA with Sammon's Mapping and FastMap with Sammon's Mapping. The same occurs for the Mahalanobis distance, i.e. the three techniques ranked best in the stress function also achieve the best neighborhood preservation behavior.

All in all, the layout created with FastMap with Force Scheme and the Mahalanobis distance attained the best stress and better neighborhood preservation, and was selected as the initial layout of the representatives to compute the particle streamfeatures for this data set. A similar comparative analysis is conducted for other data set. Streamfeature visualizations generated for different simulations are introduced and discussed in Sections 5 and 6.

\section{Visualization Framework}

The framework developed integrates four distinct views to assist interpretation of temporal data. Two of them are the streamfeature and streampath views, 
both illustrated in Figures 8 and 12, in Section 6, for two distinct simulations. The heavy density of lines makes it difficult to interact directly with those views, however. We employ multidimensional projections of both the streamfeatures and the streampaths as complementary views to support user interaction, as illustrated in Figure 7. Users may interact with those views to identify and select groups of streamfeatures or streampaths for further inspection, as illustrated in the case studies discussed in Section 6. The projections convey similarity relations over the temporal domain and, as such, help identification of temporal clusters and trends. They also afford interactions such as selection of one or multiple streamfeatures or streampaths. All four views are linked, so that interaction in a projection view, e.g., a selection, reflects in the remaining views.

Projecting the streamfeatures or streampaths requires describing them as multidimensional feature vectors. As our goal is to highlight paths with similar global and local shapes and geometric properties - e.g., starting point, size - so as to reveal similar behavior in feature space, features must be chosen that encode such information. Following the same rationale adopted by (Poco et al., 2012), our feature vectors are formed by low and high frequency coefficients of the Fast Fourier Transform (FFT) of the 3D line (Reddy and Chatterji, 1996), in each principal direction $(x, y, z)$, plus the line's initial and final positions, its size and its center of mass.

The resulting vector is described by 40 attributes: 30 Fourier coefficients (15 low-frequency in $x, y$ and $z$; 15 high-frequency in $x, y$ and $z$,) plus 10 geometrical coefficients, namely the $(x, y, z)$ coordinates of the starting point, idem for the final point and the center of mass point, plus the line size. This choice places a dominance of shape attributes (Fourier coefficients) over the geometric ones. A more balanced distribution of shape and geometric attributes may be desirable depending on the goals, e.g., if the goal is to favour perception of similar shaped streamfeatures in nearby regions. It is thus possible to vary the weights assigned to the features prior to projection. In the examples discussed in Section 6 the line features were projected with LAMP.

Users may select individual elements or groups in the projection views, and track the selection in the streampath and streamfeature views. They can manually identify groups of particles with similar behavior by delimiting visual clusters in the projection, or alternatively an $X$-means clustering (Pelleg and Moore, 2000) may be applied to identify clusters automatically. This is an extension of $k$-means clustering that finds the optimal number of clusters that best models
Table 1: Data attributes in mini dump simulation.

\begin{tabular}{|c|c|c|}
\hline Id & Attribute & Description \\
\hline 1 & radius & $\begin{array}{l}\text { particle radius of influence. It is } \\
\text { roughly inversely proportional to the } \\
\text { local density, and defines which par- } \\
\text { ticles interact with a considered par- } \\
\text { ticle. It is not a physical value. }\end{array}$ \\
\hline 2 & rho & density value at the particle's position \\
\hline 3 & temp & temperature at the particle's position \\
\hline 4 & $\begin{array}{l}\text { artificial } \\
\text { viscosity }\end{array}$ & $\begin{array}{l}\text { Used in the simulation to damp out } \\
\text { oscillations close to shocks. This is } \\
\text { not a physical quantity, but an arti- } \\
\text { ficial term in equations to mimic the } \\
\text { correct physics. }\end{array}$ \\
\hline
\end{tabular}

the data, within a give range. Once obtained, clusters are color coded in the projection, as illustrated in Figure 7. Users may select one or multiple clusters and inspect their behavior in the streampath or streamfeature views. The ability to interact with groups is particularly useful, as the streamfeature and streampath views are typically very cluttered. Users may also choose to observe behavior over a time range, rather than over the whole simulation time.

\section{Exploring Simulation Data}

We illustrate the techniques on two data sets derived from Smoothed Particle Hydrodynamics (SPH) simulations (Linsen et al., 2011) and made available by collaborators, identified as the mini dump and the data explode data sets. Both simulations were conducted to investigate the merging and/or explosion of stars in astrophysics.

\subsection{Mini Dump}

The mini dump simulation tracks 2.5 million particles over 59 time steps - we downsampled the original data set to 125,000 particles per volume. Each particle is described by four attributes, listed in Table 1 .

As discussed in Section 4, the streamfeatures for this data have been created using Fastmap with Force Scheme to project the representatives, LAMP and the Mahalanobis distance. The streamfeatures view relative to the whole simulation is shown in Figure 6(a), whereas 6(b) highlights the multidimensional axes describing the streamfeature space (not clearly visible in the upper figure, due to the high line density). In this and in the following figures that adopt the rainbow scale, color mapping indicates time evolution: blue corresponds to initial stages of the simulation, and red indicates final stages. The Cartesian 


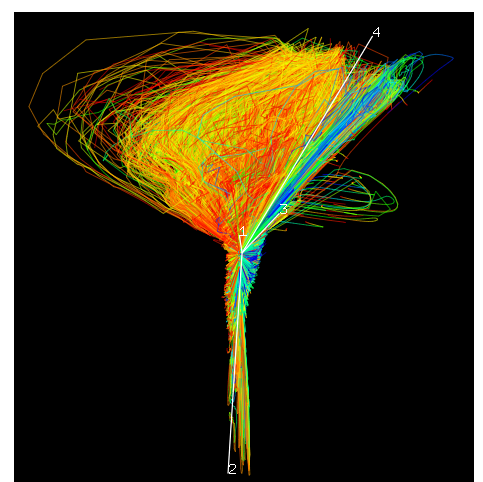

(a) Streamfeatures view.

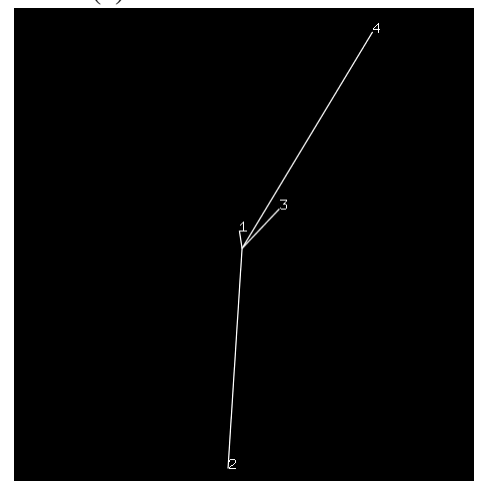

(b) Axes in streamfeature space.

Figure 6: Streamfeatures for the whole simulation (a) and axes in 4-dimensional attribute space (b) (mini dump simulation).

axes in the four-dimensional attribute space have undergone the same projection transformation applied to the data, and provide a visual hint of the attributes undergoing dominant changes in the temporal domain and the direction of change - from the figure one observes that attributes rho (axis 2) and av (axis 4)are dominant in the temporal domain.

The streamlines and streampaths were projected with LAMP using the Mahalanobis distance, but the resulting layouts are very dense and cluttered, hampering perception of groups of similar elements. The result of applying an $X$-means clustering to both projections can be seen in Figures 7 (a) and (b), which depict the 5 clusters identified - we input a range $[5,50]$ for the number of clusters to be sought for by $X$-means. Figure 7 (c) shows the streampaths feature projection view with the clusters identified: again $X$ means found 5 clusters, within a range $[5,50]$. We computed the silhouette coefficients of the resulting clusterings. This is a measure of cluster quality (Tan et al., 2005) that takes values in the range $[-1.0,1.0]$, where values closer to 1.0 indicate well separated and highly cohesive groups. Silhouette values of 0.416 and 0.799 were obtained for the streampath and

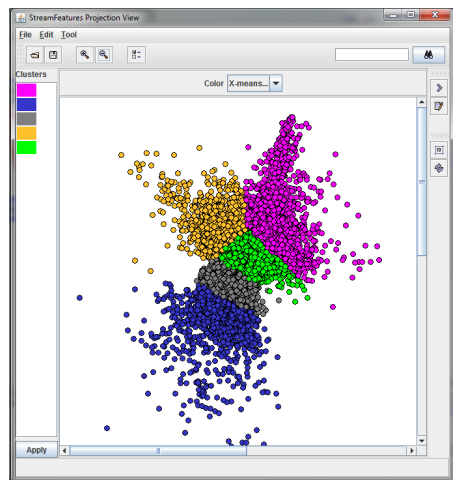

(a) Streamfeature feature projection view.

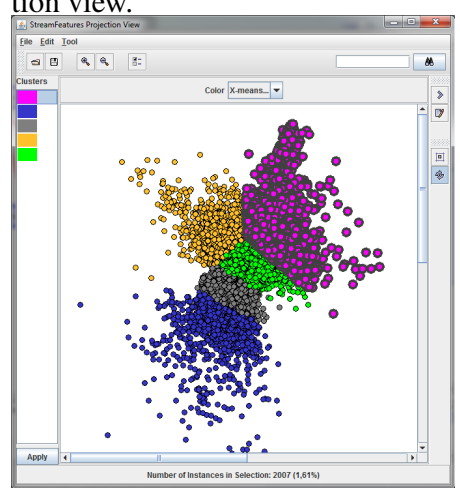

(b) Selected cluster highlighted.

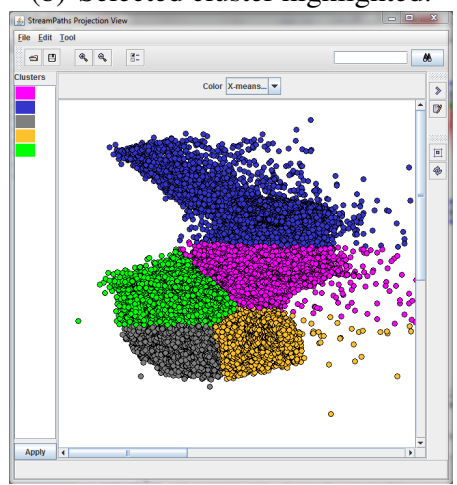

(c) Streampath feature projection view.

Figure 7: $X$-means clusters in the projection views. The pink cluster in (a) has been selected and its elements are shown highlighted (thicker borders) in (b). (c) depicts the clusters identified in the projection of the streampath features.

the streamfeature clusters, respectively. The second value indicates that the 5 clusters are well-formed, i.e., they group similar-shaped streamfeature lines, as measured by the Mahalanobis distance. Thus, they depict similar particle trajectories in feature space.

Figure 8 shows how the particles in the selected pink cluster in Figure 7(b) evolve in the simulation, 


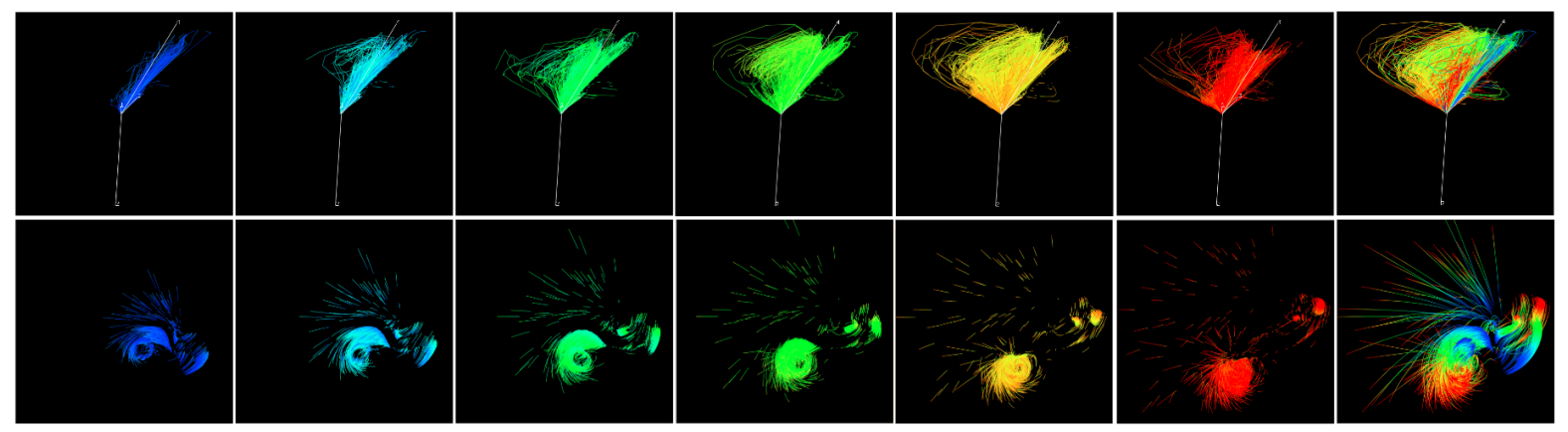

Figure 8: Mini dump simulation: top row images show the streamfeatures describing the behavior of a selected group of particles, bottom row images show the corresponding streampaths. The rightmost images refer to the whole simulation (59 time steps), whereas the previous ones show streamfeatures and streampaths computed over a sequence of time windows of size 10, namely 0-10, 10-20, 20-30, 30-40, 40-50 and 50-59. Most particles are moving towards the center of the two stars, and one observes little variation happening in the direction associated to attribute rho (axis 2, the vertical line going down in the central region).

with blue to red mapping time evolution. This particular cluster includes 2,007 particles, or $1,61 \%$ of the total number of particles tracked. The top row shows images of the streamfeatures and the bottom row show the corresponding streampaths, computed over different simulation time intervals. Interpreting a visualization that covers the whole temporal domain is not straightforward, it may thus be interesting to observe behavior over shorter time intervals, e.g., as shown in the figure, over windows of size 10, corresponding to 10 simulation time steps.

The streampaths in Figure 8 indicate that particles in this group are being attracted to the center of the two stars. In the streamfeature views we notice that major changes occur in the directions associated to attributes radius (axis 1), temperature (axis 3 ) and artificial viscosity (axis 4), while no major changes are observed in the direction associated to attribute rho (axis 2) for this particular cluster. Furthermore, notice that changes were initially more localized around the direction defined by attribute artificial viscosity (4), but then from time interval 40-50 the attributes radius (1) and temperature (3) start to also play a more dominant role in particle behavior. Figure 9 shows rotated views of the rightmost views in Figure 8, illustrating that users can interact with these $3 \mathrm{D}$ representations while exploring the data.

\subsection{Data Explode}

This data set describes the behavior of 39,200 particles over 200 time steps. Although each particle is originally described by 13 attributes, some scalar fields are known to be highly correlated. We thus preserved a smaller subset of 9 attributes to create the visualizations, listed in Table 2.

The streamfeatures have been generated employ-

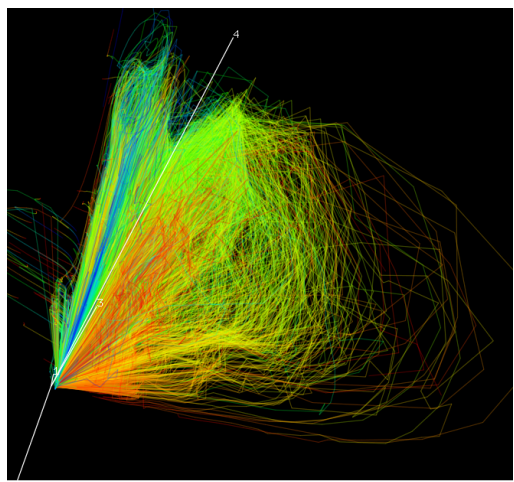

(a) Streamfeatures view.

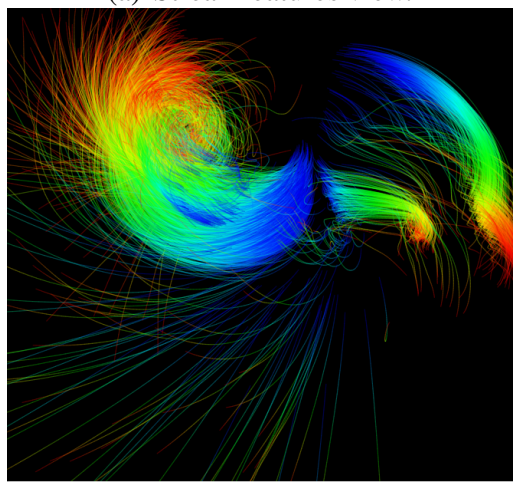

(b) Streampaths view

Figure 9: Rotated views of the visualizations shown in the last column of Figure 8 (mini-dump simulation).

ing FastMap with Force Scheme, LAMP and the Mahalanobis distance as the dissimilarity function, after considering several alternatives and conducting an analysis similar to that detailed for the mini dump data. The resulting streamfeatures computed over the whole simulation, and the projected axes of the 9dimensional feature space are shown, respectively, in 
Table 2: Particle attributes in data explode simulation.

\begin{tabular}{|l|l|l|}
\hline Id & Attribute & Description \\
\hline 1 & radius & $\begin{array}{l}\text { radius of influence of a particle. } \\
\text { It is roughly inversely propor- } \\
\text { tional to the local density } \\
\text { fines which particles interact with } \\
\text { a considered particle. It is not a } \\
\text { physical value }\end{array}$ \\
\hline 2 & $\begin{array}{l}\text { internal } \\
\text { energy }\end{array}$ & internal energy \\
\hline 3 & Temperature & $\begin{array}{l}\text { temperature at the particle's posi- } \\
\text { tion }\end{array}$ \\
\hline 4 & helium4 & an isotope of helium $\left({ }_{4}^{2} \mathrm{He}\right)$ \\
\hline 5 & carbon12 & an isotope of carbon $\left({ }^{12} \mathrm{C}\right)$ \\
\hline 6 & neon20 & an isotope of neon $\left({ }^{20} \mathrm{C}\right)$ \\
\hline 7 & magnesium24 & an isotope of magnesium $\left({ }^{24} \mathrm{C}\right)$ \\
\hline 8 & silicon-group & silicon-group \\
\hline 9 & abar & abar \\
\hline
\end{tabular}

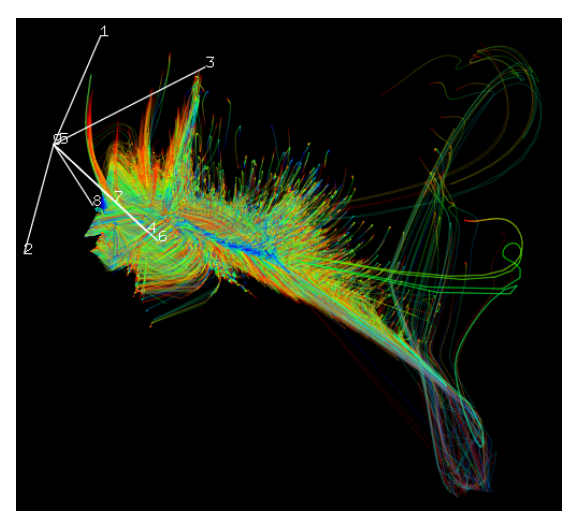

(a) Streamfeatures view.

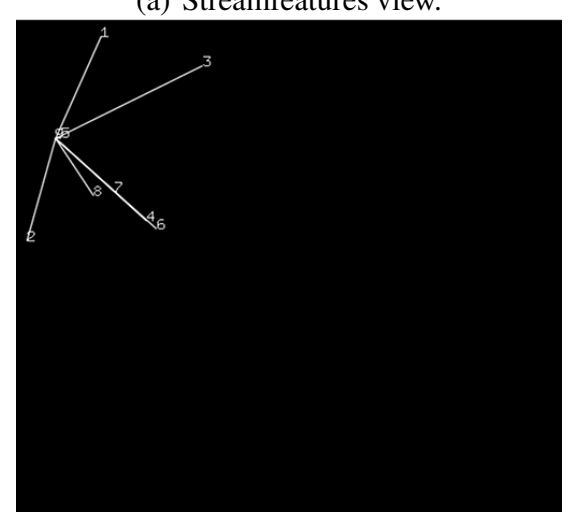

(b) Axes describing streamfeature space.

Figure 10: Streamfeatures computed over the whole simulation (a) and axes in 9-dimensional attribute space (data explode simulation).

Figures 10(a) and 10(b).

The projection of the features describing the streamfeatures computed with LAMP and Mahalanobis appears in Figure 11, with extracted clusters color coded. $X$-Means identified 7 clusters as the best

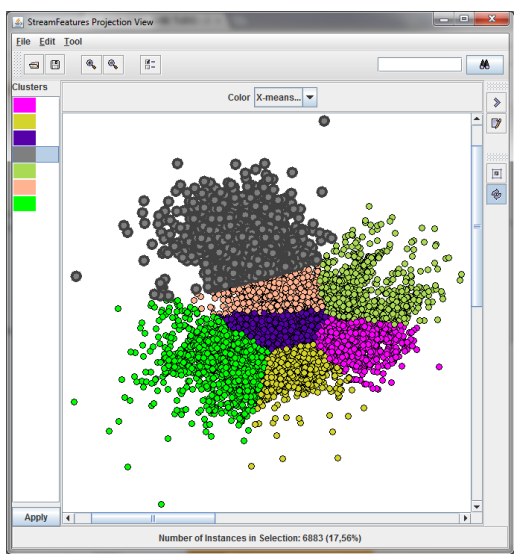

Figure 11: $X$-means clusters in the streamfeature feature projection. The gray cluster has been selected and its elements are shown highlighted.

configuration (on a range $[5,50]$ ). The silhouette coefficient computed for this result was 0.482 , indicating clusters not as well-formed as in the previous example.

Figure 12 shows the evolution of the cluster selected in Figure 11, which includes 6,883 particles, $17,56 \%$ of the total number. Images in the top row depict the particle streampaths, and in the bottom row the corresponding streamfeatures are shown. One notices that some particles in this group are trapped in the gravitational field of the star, those seen in the central circular shape in the streampath views, whereas others seem to escape the star attraction and are lost to space, shown by the streampaths tangent to the circular shape. As the simulation starts the major directions of change seem to be associated with attributes Helium, Neon and Magnesium (axes 4, 6 and 7, respectively), as it evolves attributes radius and temperature seem to start playing a major role (notice the outgoing streamfeature lines on the right region of the streamfeature views).

Figure 13 illustrates an interface for users to filter the information displayed based on streampath length, by setting lower and upper length thresholds. This allows them to focus, e.g., on analyzing a sub-set of particles with greater (or smaller) displacement in the object space. Visualizations after such filtering are illustrated in Figure 14: they cover the whole simulation, but shown only lines that survived the filtering. Each column shows a view of the streampaths (top) and the corresponding streamfeatures (bottom).

From left to right, images in the first column display the object and feature space behavior of 3 particles, then 12, 441 and 1,880 particles, respectively: the first three views show only particles with greater displacement, whereas the last one shows particles 


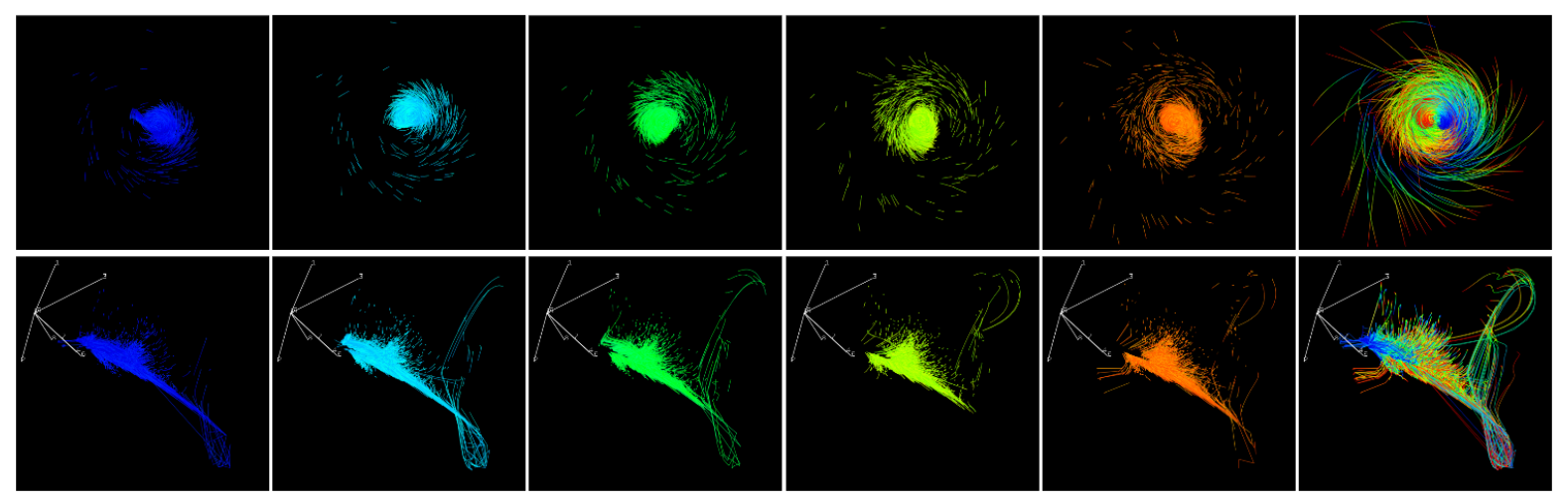

Figure 12: Streampaths (top) and streamfeatures (bottom) of a selected cluster of particles in the data explode simulation. The rightmost images display the streamfeatures and streampaths computed over the whole simulation (200 time steps). A sequence of streamfeatures and streampaths relative to time slices of size 20, namely $0-20,40-60,80-100,120-140$ and 160-180, is shown from left to right.

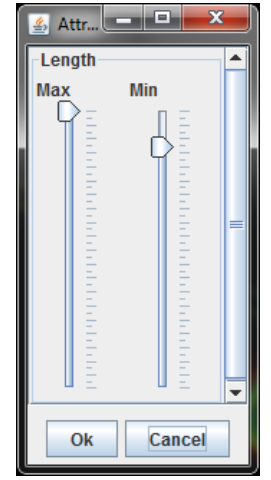

(a)

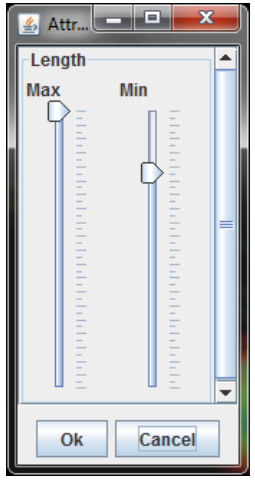

(b)

Figure 13: Filtering streampaths based on their length: setting lower and upper thresholds (data explode simulation).

with smaller displacement. One observes that most particles show small spatial displacement, highly concentrated in the central region, whereas the 3 particles with greater displacement wander away from this central area.

Behavior of the 1,880 particles with low spatial displacement in the simulation is better observed in the views in Figure 15.

Figure 16 shows visualizations of the projections of the features extracted from streampaths and the streamfeatures, highlighting the particles filtered.

\section{Conclusions}

We described a framework developed to support the exploratory visualization of data generated by multivariate particle simulations. This is a challenging problem, as it requires handling a temporal sequence of multi-attribute volumes and dealing with

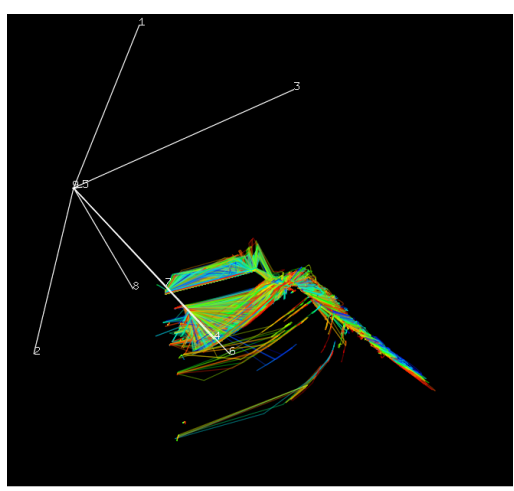

(a) Streamfeatures.

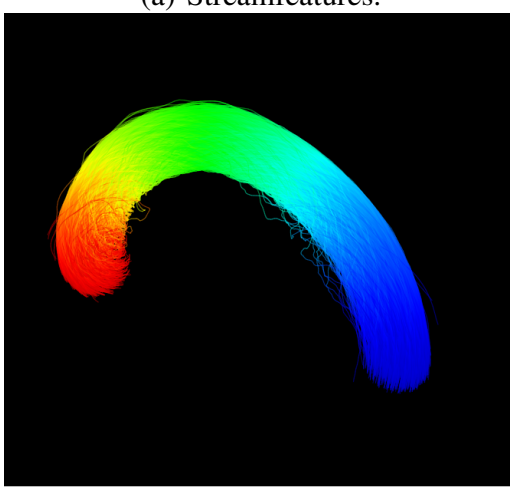

(b) Streampaths.

Figure 15: Observing behavior of 1,880 particles filtered based on their spatial displacement: particles with small displacement.

issues related to huge data set sizes and interpreting time-varying multidimensional information.

The proposed framework integrates visualizations of both the spatial (physical) and the multidimensional feature space domains, and includes functionalities that help users to observe and interpret particle 


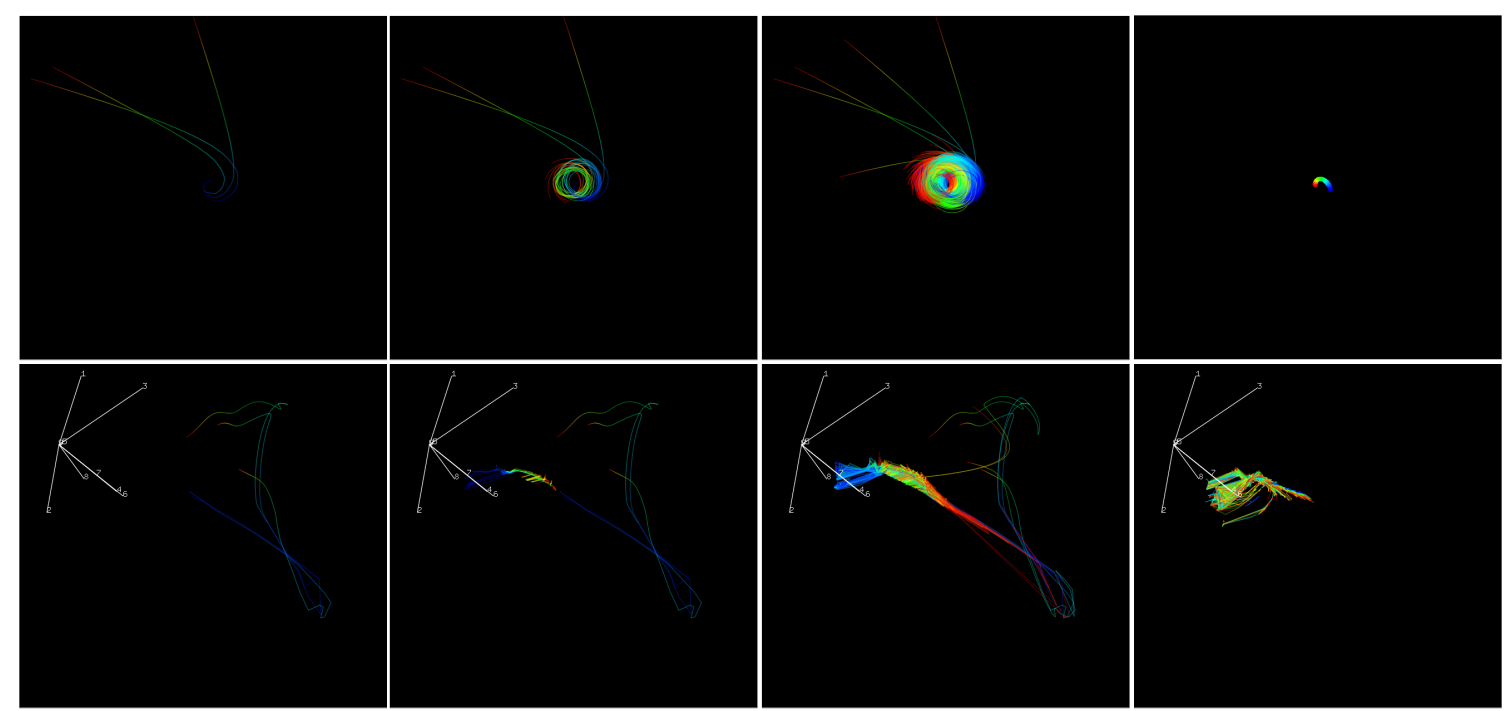

Figure 14: Each column shows a view of streampaths (top) and streamfeatures (bottom) filterered by their length. From left to right, images in the first column display the object and feature space behavior of 3 particles, then 12, 441 and 1,880 particles, along the data explode simulation.

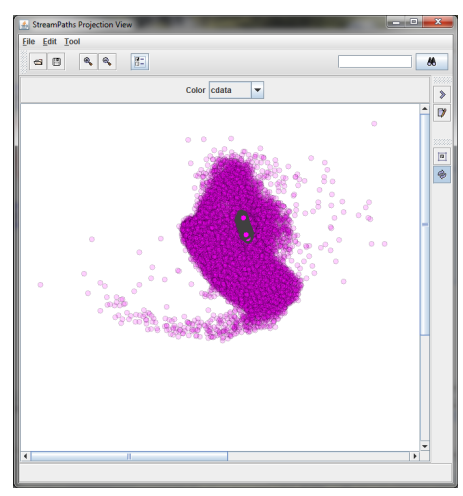

(a) Streampath feature projection.

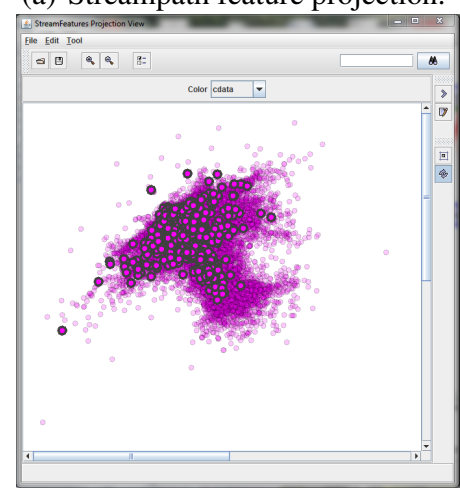

(b) Streamfeature feature projection.

Figure 16: Projections of the streamfeature and streampath features: 1,880 low-displacement particles filtered are shown highlighted. behavior in both spaces simultaneously, over the temporal domain. This is achieved by combining multiple visualizations to assist exploration of the time-varying particle volumes. Coordinated views of both feature and physical spaces allow the observation of particle behavior over the whole temporal domain or over selected time windows, rather than restricting users to handle a single simulation time step.

The proposed feature space visualization relies on an analog of the well-known streamlines for visualizing spatial behavior of fluid. Like most volume visualization techniques, the streamfeatures also suffer with overcrowding of the visual space as the number of particles increases. This fact may be quickly aggravated when handling simulations that involve a large number of time steps. This is handled with proper interaction filters to cluster and filter the visualizations. Usage of the framework has been illustrated on data generated by two simulations in astrophysics. As further work we intend to validate the framework in other data sets, in cooperation with domain experts.

\section{Acknowledgments}

Authors acknowledge the financial support of FAPESP, CNPq and CAPES-PROBRAL. We are grateful to Lars Linsen and Vladimir Molchanov, from the Visualization and Computer Graphics Laboratory at Jacobs University, Bremen, for making available the simulation data. 


\section{REFERENCES}

Blaas, J. and Post, C. P. B. F. (2008). Extensions of parallel coordinates for interactive exploration of large multitimepoint data sets. IEEE Transactions on Visualization and Computer Graphics, 14(6):1436-1451.

Co, C. S., Friedman, A., Grote, D. P., Vay, J.-L., Bethel, Wes, E., and Joy, K. I. (2004). Interactive methods for exploring particle simulation data. Lawrence Berkeley National Laboratory: Lawrence Berkeley National Laboratory. Retrieved from: http://escholarship.org/uc/item/0v36500n.

Falk, M., Grottel, S., and Ertl, T. (2010). Interactive imagespace volume visualization for dynamic particle simulations. In SIGRAD.

Faloutsos, C. and Lin, K.-I. (1995). FastMap. In Proceedings of the 1995 ACM SIGMOD international conference on Management of data - SIGMOD '95, pages 163-174, New York, New York, USA. ACM Press.

Gribble, C. P., Stephens, A. J., Guilkey, J. E., and Parker, S. G. (2006). Visualizing particle-based simulation datasets on the desktop. In British HCI Workshop on Combining Visualization and Interaction to Facilitate Scientific Exploration and Discovery, pages 111-118.

Inselberg, A. (1985). The plane with parallel coordinates. The Visual Computer, 1(2):69-91.

Joia, P., Paulovich, F. V., Coimbra, D., Cuminato, J. A., and Nonato, L. G. (2011). Local Affine Multidimensional Projection. IEEE transactions on visualization and computer graphics, 17(12):2563-71.

Jolliffe, I. T. (2002). Principal Component Analysis. Springer.

Jones, C., Ma, K.-L., Ethier, S., and Lee, W.-L. (2008). An integrated exploration approach to visualizing multivariate particle data. Computing in Science and Engineering, 10(4):20-29.

Kruskal, J. B. (1964). Multidimensional scaling by optimizing goodness of fit to a nonmetric hypothesis. Psychometrika, 29(1):1-27.

Linsen, L., Long, T. V., and Rosenthal, P. (2009). Linking multi-dimensional feature space cluster visualization to surface extraction from multi-field volume data. IEEE Computer Graphics and Applications, 29(3):85-89.

Linsen, L., Molchanov, V., Dobrev, P., Rosswog, S., Rosenthal, P., and Long, T. V. (2011). Hydrodynamics - Optimizing Methods and Tools, chapter SmoothViz: Visualization of Smoothed Particles Hydrodynamics Data. inTech. Available from: http://www.intechopen.com/books/hydrodynamicsoptimizing-methods-and-tools/smoothvizvisualization-of-smoothed-particles-hydrodynamicsdata.

Linsen, L., Van Long, T., Rosenthal, P., and Rosswog, S. (2008). Surface extraction from multi-field particle volume data using multi-dimensional cluster visualization. IEEE transactions on visualization and computer graphics, 14(6):1483-90.

Paulovich, F. V., Nonato, L. G., Minghim, R., and Levkowitz, H. (2008). Least square projection: a fast high-precision multidimensional projection technique and its application to document mapping. IEEE transactions on visualization and computer graphics, 14(3):564-75.

Paulovich, F. V., Silva, C. T., and Nonato, L. G. (2010). Two-phase mapping for projecting massive data sets. IEEE transactions on visualization and computer graphics, 16(6):1281-90.

Pelleg, D. and Moore, A. (2000). X-means: Extending k-means with efficient estimation of the number of clusters. In Proceedings of the Seventeenth International Conference on Machine Learning, pages 727 734. San Francisco.

Poco, J., Eler, D., Paulovich, F., and Minghim, R. (2012). Employing 2d projections for fast visual exploration of large fiber tracking. Computer Graphics Forum, 31(3):1075-1084.

Poco, J., Etemadpour, R., Paulovich, F., Long, T., Rosenthal, P., Oliveira, M., Linsen, L., and Minghim, R. (2011). A Framework for Exploring Multidimensional Data with 3D Projections. Computer Graphics Forum, 30(3):1111-1120.

Reddy, B. S. and Chatterji, B. N. (1996). An FFT-based technique for translation, rotation, and scale-invariant image registration. IEEE transactions on image processing : a publication of the IEEE Signal Processing Society, 5(8):1266-71.

Tan, P.-n., Steinbach, M., and Kumar, V. (2005). Introduction to Data Mining. Addison Wesley, Boston, MA

Tejada, E., Minghim, R., and Gustavo Nonato, L. (2003). On improved projection techniques to support visual exploration of multi-dimensional data sets. Information Visualization, 2(4):218-231.

Wei, J., Yu, H., Grout, R., Chen, J., and Ma, K.-L. (2012). Visual analysis of particle behaviors to understand combustion simulations. IEEE Comput. Graph. Appl., 32(1):22-33. 